\title{
EFFECT OF pH AND TEMPERATURE ON THE BINDING INTERACTIONS OF CAFFEINE AND CHLOROGENIC ACID WITH SODIUM CASEINATE
}

\author{
A Thesis \\ presented to \\ the Faculty of California Polytechnic State University, \\ San Luis Obispo
}

\begin{abstract}
In Partial Fulfillment
of the Requirements for the Degree

Master of Science in Agriculture, with Specialization in Dairy Product Technology
\end{abstract}

By

Emily S. Kong

May 2013 
(C) 2013

Emily S. Kong

ALL RIGHTS RESERVED

Page ii 


\section{COMMITTEE MEMBERSHIP}

TITLE:

AUTHOR:

DATE SUBMITTED:

COMMITTEE CHAIR:

COMMITTEE MEMBER:

COMMITTEE MEMBER:
EFFECT OF $\mathrm{pH}$ AND TEMPERATURE ON THE BINDING INTERACTIONS OF CAFFEINE AND CHLOROGENIC ACID WITH SODIUM CASEINATE

Emily S. Kong

May 2013

Dr. Rafael Jimenez-Flores, PhD., Professor Department of Dairy Science California Polytechnic State University San Luis Obispo

Dr. Margaret Rice, PhD., Professor Department of Chemistry \& Biochemistry California Polytechnic State University San Luis Obispo

Dr. Phillip S. Tong, PhD., Professor Department of Dairy Science California Polytechnic State University San Luis Obispo 


\section{ABSTRACT \\ EFFECT OF pH AND TEMPERATURE ON THE BINDING INTERACTIONS OF CAFFEINE AND CHLOROGENIC ACID WITH SODIUM CASEINATE \\ Emily S. Kong}

Coffee is a popular and well-loved beverage consumed worldwide by millions of people every day. While most patrons of coffee do so because of its unique and satisfying taste, consumers may be unaware of the potential beneficial health effects it also imparts. The antioxidants found in green coffee beans collectively known as chlorogenic acids (CGA) and caffeine are two of the most abundant bioactive compounds present in coffee. Both these bioactive compounds have been implicated in many studies to impart a wide range of health benefits, from reducing the risk of Type 2 diabetes, to their use as aides in weight management. Indeed, epidemiological studies on people who consume moderate amounts of coffee on a regular basis have unanimously shown benefits to overall health.

While caffeine and CGA are naturally occurring compounds in coffee, their potential in conferring beneficial health effects warrant research into other potential food matrixes that can be used to bind and deliver these bioactive compounds into foods that do not naturally contain them. Milk proteins, specifically caseins, have been shown to be excellent vehicles to both bind and deliver sensitive bioactive compounds of various chemical and physical properties. Caseins have been shown in numerous studies to successfully bind to 
molecules such as vitamin $\mathrm{D}_{2}, \omega-3$ polyunsaturated fatty acids (DHA), and iron to name a few. Because caseins exhibit high versatility in binding a variety of molecules, caseins were the milk protein of choice for the experiments in this thesis.

Polyphenols have been the subject of many studies on its binding capacity with milk proteins, but research on the binding capacity of caffeine with caseins is limited. Therefore, the objectives of this thesis are threefold: 1) develop, optimize and validate an HPLC method for the accurate and simultaneous determination of caffeine and CGA, 2) establish a procedure by which caffeine and CGA bind to sodium caseinate, and 3) determine the optimal treatment conditions of $\mathrm{pH}$ and temperature to increase binding interactions and speculate on the mechanism of binding for each bioactive compound.

A reversed phase HPLC (RP-HPLC) method was developed and subjected to validation studies with good results in linearity (caffeine $\mathrm{R}^{2}=0.9992$, CGA $\left.\mathrm{R}^{2}=0.9995\right)$ and precision ( $\mathrm{RSD}$ of caffeine $<1 \%$, RSD of CGA $<2 \%$ ). The developed method also demonstrated selectivity for caffeine and CGA. This method was then used to analyze sodium caseinate samples containing caffeine and 5-CGA. The results from these studies have shown that binding interactions between caffeine and sodium caseinate are temperature dependent $(\mathrm{p}<0.01)$ whereas binding interactions between CGA and sodium caseinate are influenced by both $\mathrm{pH}$ and temperature $(\mathrm{p}<0.01)$. Elucidating the binding mechanisms of caffeine and CGA to sodium caseinate and providing a sensitive analytical technique by which these compounds can be accurately quantified may facilitate 
future research involving the use of caffeine and CGA in many other facets, as well as promoting its increased use in the dairy industry. 


\section{ACKNOWLEDGMENTS}

I wish to thank my committee chair and advisor, Dr. Jimenez-Flores for his guidance and support. Thank you for giving me the opportunity to learn and grow at Cal Poly. I would like to thank Dr. Peggy Rice for her guidance, advice, and fruitful conversations. Thank you for showing me the insides of an HPLC pump (many times) late at night and on weekends and showing me the enjoyment one can get from disassembling and reassembling them. I would like to thank Dr. Phillip Tong for his willingness to always share his knowledge and for his continuous support.

I would like to thank Dr. Nana Farkye, for always being positive. Thank you for your tireless words of encouragement and for your wonderful conversations. I always left your office feeling better. I must also acknowledge and thank Joseph Kuo for his generous help.

I would like to thank Dr. Wyatt Brown for generously lending us the use of his HPLC, for which this project would not have been possible without. Thank you to the graduate students in Dr. Brown's lab for the enjoyable company. Special thanks to Jim Green for all his help.

I am extremely thankful for all the incredible professors at Cal Poly who have taught me so much. A special thank you to Dr. John Walker and Dr. Karen 
McGaughey for their immeasurable dedication, passion, and enthusiasm. Thank you for tirelessly reviewing my stats outputs, and most of all, for teaching me the values of and sparking my interest in statistics. I would also like to thank Dr. Corinne Lehr for her patience and for her willingness to help with the analytical chemistry portion of this work.

To my fellow graduate students, I would like to thank: Hadi Eshpari, for his kind words of encouragement, his great sense of humor, and most of all, his perseverance. Lauren Collinsworth, for sharing almonds with me during late nights at the DPTC. Melissa Hsu, Valerie Arechiga and Corbin Kembel, my unofficial swim coaches and team UdderSuckers. Tracey Nguyen, for being my neighbor, in every sense of the word, and for going through this journey with me from the beginning. Mariela Poveda and Garrett Walsh, for the welcomed entertainment. Katy Lees, thank you for all your wonderful treats and visits. A very special thanks to Andrea Laubscher, who has always made my life easier from the start; thank you for your positive attitude, calm demeanor, and kind friendship. Your unconditional help and guidance made this thesis project possible. Many thanks also to Dan Zhao, Sean Vink, Vandna Sikand, and Kristy Herbaugh for each shaping my experience at the DPTC. I am thankful to have met and gotten to know every one of you.

To Kelly Carnes and Kathi Reis, you both have changed my life in ways you cannot imagine. Thank you for teaching me what no books could. Thank you Page viii 
Kelly, for empowering me to become a stronger person, physically and mentally. Thank you Kathi, for your patience, dedication, and constant encouragement; you have irreversibly changed my life for the better. Thank you for being strong, confident women, and inspiring me to be the same. I am forever grateful to have met you both.

To my life before Cal Poly: I would like to thank Dr. David LechugaBallesteros for being a mentor, friend and supporter since before Day 1. Thank you for providing much needed comic relief in tense situations and for your transcending guidance through trying times. I would also like to thank Jon Schroeder, to whom I owe practically all my Word \& Excel skills to; they came in very handy during the writing of this thesis. Thank you for teaching me how to be a good analytical chemist. I would like to thank Dr. Melissa Turcotte for her words of wisdom that only a recent $\mathrm{PhD}$ graduate could provide, and Dr. Nancy Carrillo for relentlessly encouraging me to pursue a Master's.

I would like to thank my parents for their endless support through every one of my seemingly impulsive decisions. Thank you for always believing that you raised a daughter with her head on straight. Thank you to my brothers and sisters-in-law: Jeff, Jerry, Tam \& Rujjira, for always answering my calls, believing in me, and for your unconditional love and support. I am lucky to call you all family. And to my little niece, Hannah, who at just 10 months old has 
provided the impetus for finishing graduate school in a timely matter so that I can watch her grow up.

I would like to thank my dear friends, Katherine Fader, Josh Marcley, Vish Patel, Ron Sloat, Randy Spagnola, Diem Tran, and Linda Y for their unconditional love and support. You guys are my strength in times of weakness.

And finally, to Michael Drew. Thank you for being my inspiration. Thank you for moving with me, for many moments of laughter, giving me perspective, letting me watch TV after a bad day, and for always being there. Most of all, thank you for vicariously living through another 2 years of graduate school. 


\section{TABLE OF CONTENTS}

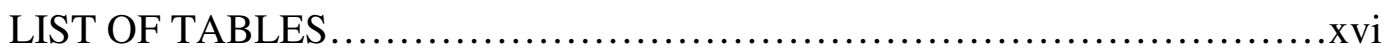

LIST OF FIGURES ................................................

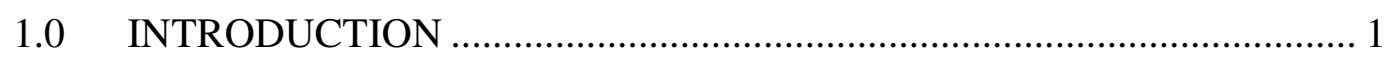

$2.0 \quad$ LITERATURE REVIEW ............................................................. 3

2.1 Milk proteins ............................................................................ 3

2.1.1 Overview of milk proteins ..................................................... 3

2.1.2 Functional milk proteins .................................................... 4

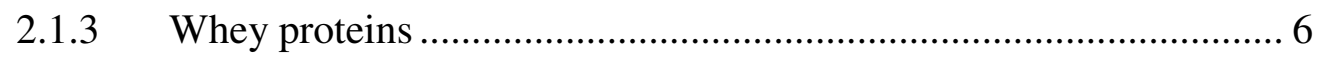

2.1.4 Milk proteins as delivery systems for bioactives ......................... 7

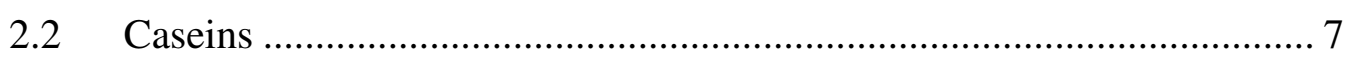

2.2.1 Chemistry of caseins .......................................................... 7

2.2.2 Physiochemical properties of the fractions of casein.................... 9

2.2.3 Structural properties of casein ............................................. 11

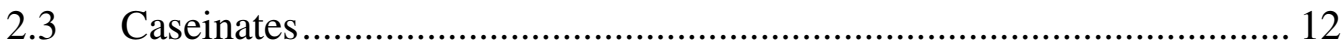

2.3.1 Preparation of sodium caseinate ............................................. 12

2.3.2 Sodium caseinate as a delivery system .................................... 13

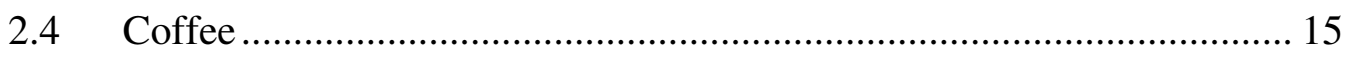

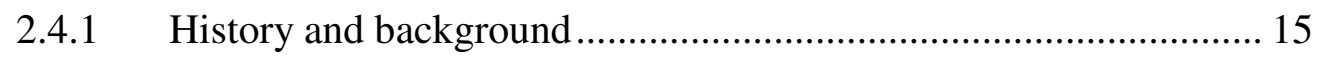

2.4.2 Composition of the coffee fruit............................................... 16

2.4.3 Processing \& effects of roasting on coffee beans ....................... 17

2.4.4 Coffee bioactives .................................................................. 19

Page xi 


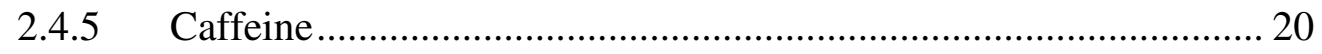

2.4.6 Chlorogenic acid (CGA) ..................................................... 22

2.5 Health benefits of coffee bioactives ............................................ 24

2.5.1 Health benefits of caffeine ........................................................ 24

2.5.2 Health benefits of chlorogenic acid ....................................... 25

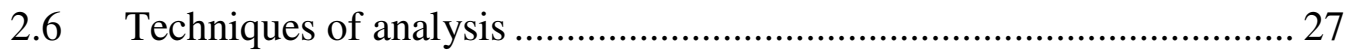

2.6.1 High Pressure Liquid Chromatography (HPLC) .......................... 28

2.6.2 Oxygen Radical Absorbance Capacity (ORAC) assay of

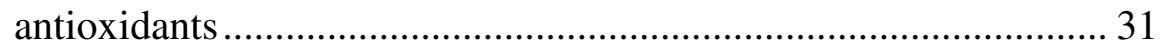

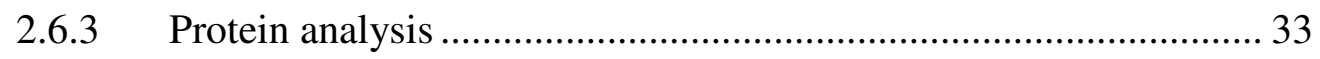

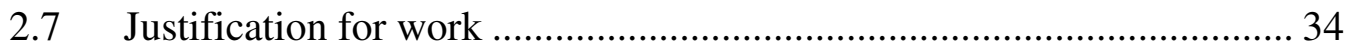

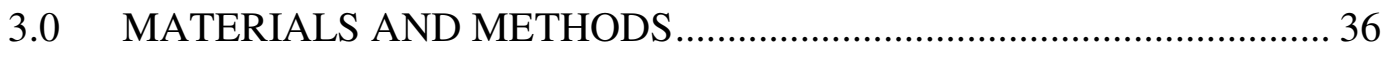

3.1 Development of a method for the simultaneous determination of caffeine and CGA by reversed phase High Pressure Liquid

Chromatography (RP-HPLC)................................................. 36

3.1.1 HPLC Method optimization for the analysis of caffeine and

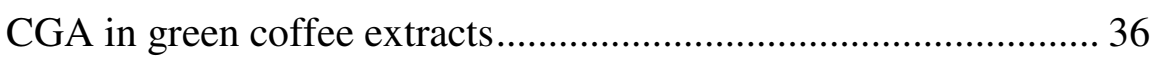

3.1.1.1 Mobile phase gradient .................................................... 36

3.1.1.2 Analytical column ........................................................ 40

3.1.1.3 Column temperature ..................................................... 41

3.1.2 Development of a method for the simultaneous determination of caffeine and CGA in green coffee extracts by RP-HPLC............. 42

3.2 HPLC Method validation studies ................................................. 44

Page xii 


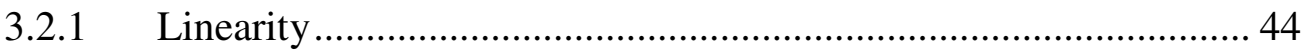

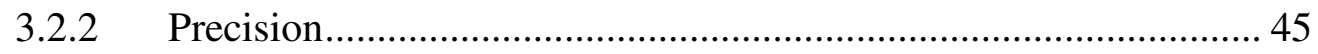

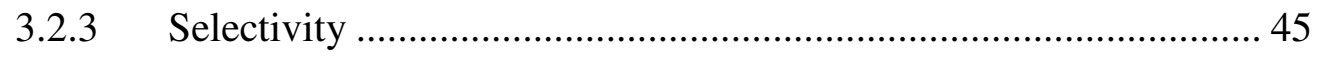

3.3 Binding interactions of caffeine and 5-CGA with sodium caseinate ..... 46

3.3.1 Establishing a procedure for binding caffeine to sodium

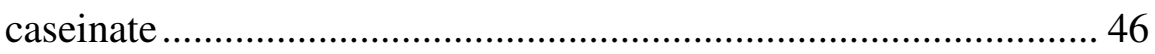

3.3.1.1 Preparation of caffeine stock standard ........................................ 46

3.3.1.2 Preparation of sodium caseinate sample …………...................... 47

3.3.1.3 Preparation of 1:1 M ratio sodium caseinate to

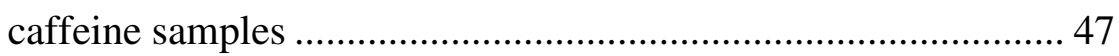

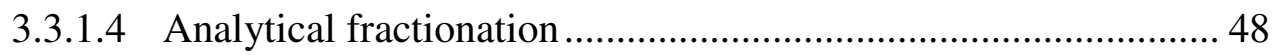

3.3.2 Establishing a procedure for binding 5-CGA to sodium

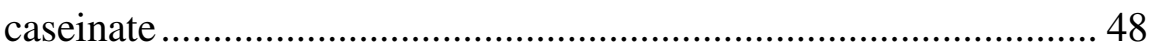

3.3.2.1 Preparation of 5-CGA stock standard ......................................... 48

3.3.2.2 Preparation of 1:1 $\mathrm{M}$ ratio sodium caseinate to 5-CGA

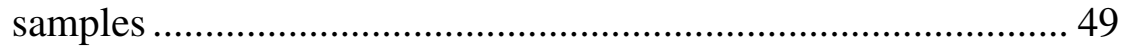

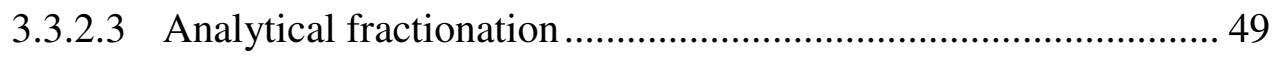

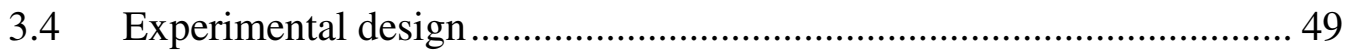

3.5 Application of established protocol and optimal treatment conditions

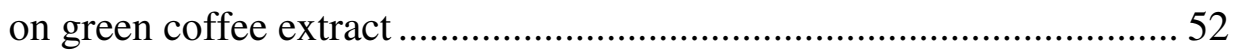

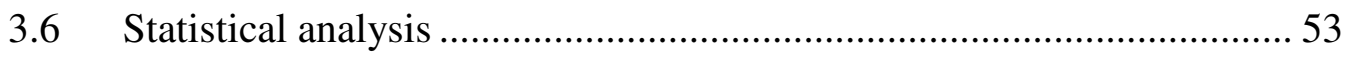

3.7 HPLC analysis of caffeine and 5-CGA ................................................ 53

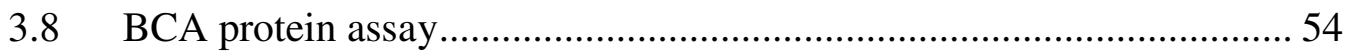

Page xiii 


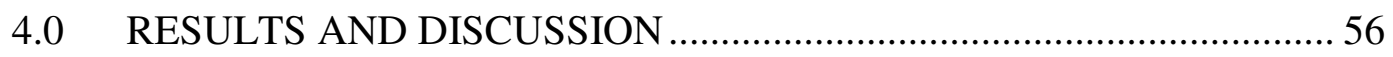

4.1 Development of a method for the simultaneous determination of caffeine and CGA in green coffee extracts by RP-HPLC ....................... 56

4.1.1 Determination of HPLC chromatographic conditions ..................... 56

4.1.1.1 Gradient profile ........................................................................ 56

4.1.1.2 Analytical column ............................................................... 58

4.1.1.3 Column temperature ................................................................. 59

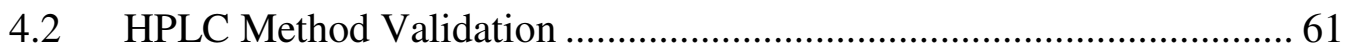

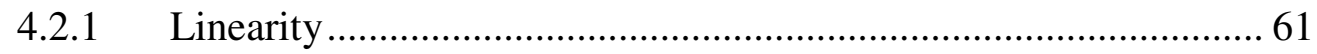

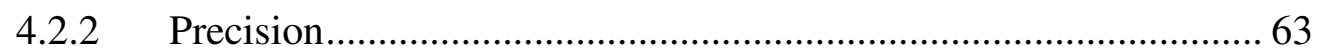

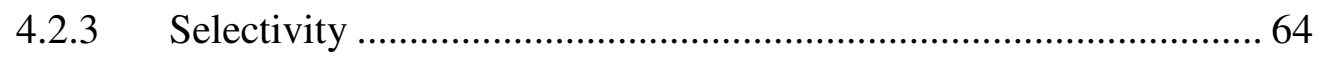

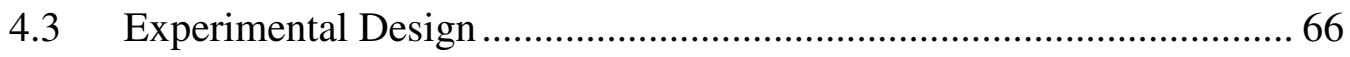

4.4 Binding caffeine \& 5-CGA to sodium caseinate....................................... 69

4.4.1 Binding caffeine to sodium caseinate trials .................................... 69

4.4.2 Binding 5-CGA to sodium caseinate trials .................................... 74

4.4.3 Binding caffeine \& 5-CGA present in green coffee extract to sodium caseinate trials ................................................................. 77

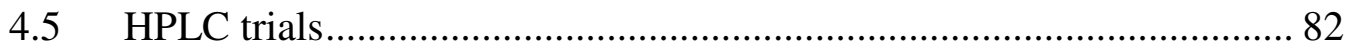

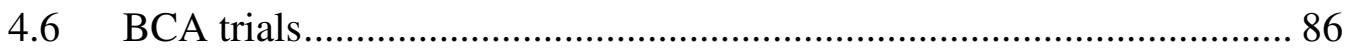

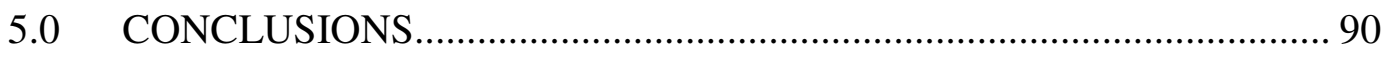

6.0 DIRECTIONS FOR FUTURE RESEARCH........................................... 94

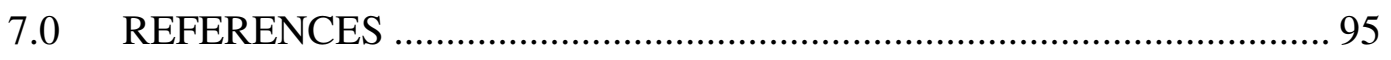

Page xiv 
APPENDIX A: Data for precision studies on caffeine and 5-CGA............102

APPENDIX B: Statistical analysis by JMP - binding caffeine to sodium

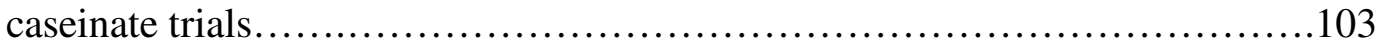

APPENDIX C: Statistical analysis by JMP - binding 5-CGA to sodium

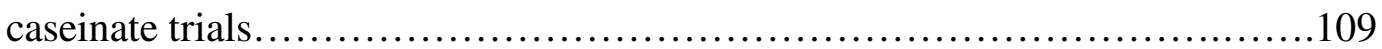

APPENDIX D: Statistical analysis by JMP - model assumptions (caffeine)...115

APPENDIX E: Statistical analysis by JMP - model assumptions (5-CGA).....117 


\section{LIST OF TABLES}

Table 2.1. Composition $\%$ of milks of some species ...................................... 4

Table 2.2. Composition of casein and caseinates ......................................... 5

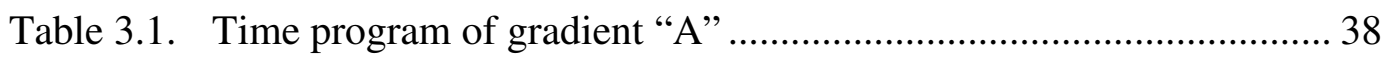

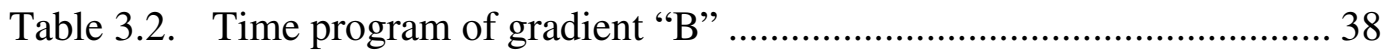

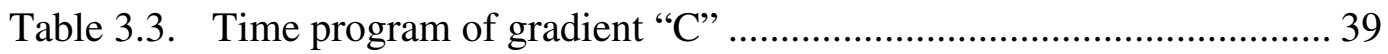

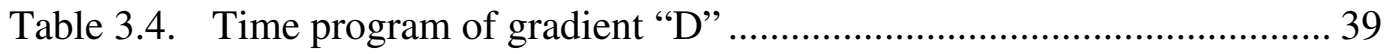

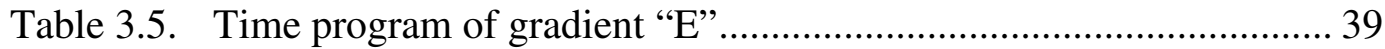

Table 3.6. Time program of final gradient profile........................................ 40

Table 3.7. Levels of the treatment factors …............................................... 50

Table 3.8. Randomization of treatments to experimental unit in caffeine

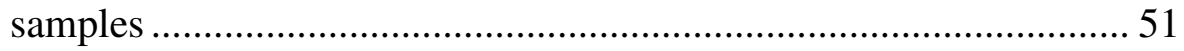

Table 3.9. Randomization of treatments to experimental unit in 5-CGA

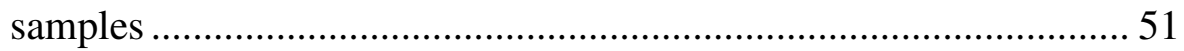

Table 3.10. Randomly selected samples and their treatment combinations......... 55

Table 4.1. Concentration range and peak areas of caffeine and 5-CGA used

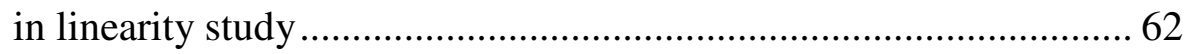

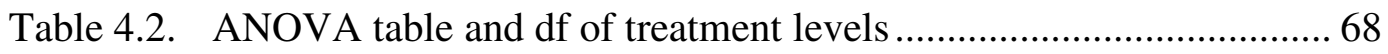

Table 4.3. Retention times and relative retention times of caffeine and CGA

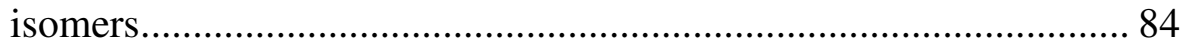

Table 4.4. BCA protein assay of 1:1 M sodium caseinate:caffeine samples ..... 88

Table 4.5. BCA protein assay of 1:1 $\mathrm{M}$ sodium caseinate:5-CGA samples....... 88 Page xvi 


\section{LIST OF FIGURES}

Figure 2.1. Cryo-TEM tomograms of casein micelles ................................. 12

Figure 2.2. Layers in a coffee fruit ...................................................... 17

Figure 2.3. Chemical structures of cafestol and kahweol.............................. 20

Figure 2.4. Chemical structures of caffeine and adenosine ............................ 22

Figure 2.5. Chemical structure of 5-CGA …............................................... 24

Figure 2.6. TEM images of hIAPP co-incubated with different compounds..... 26

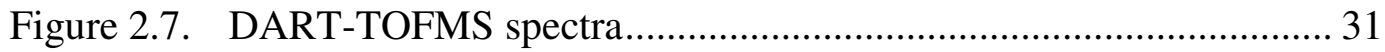

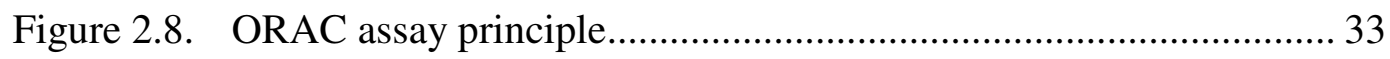

Figure 3.1. HPLC chromatogram of caffeine analysis $(280 \mathrm{~nm})$ from a green

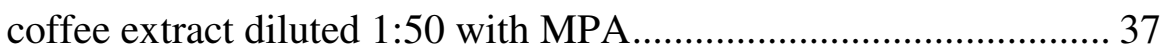

Figure 3.2. Gradient profiles for separation of caffeine by HPLC .................... 40

Figure 3.3. HPLC analytical columns ................................................... 41

Figure 3.4. Shimadzu Prominance HPLC unit ........................................... 44

Figure 3.5. Permeate of green coffee extract obtained after ultrafiltration ........ 53

Figure 4.1. Overlay of green coffee extract.............................................. 57

Figure 4.2. Slight peak sharpening observed in caffeine peak....................... 57

Figure 4.3. Overlay of green coffee extract.............................................. 58

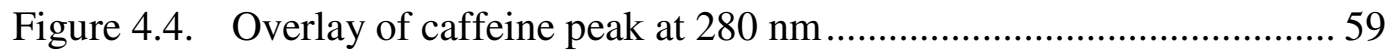

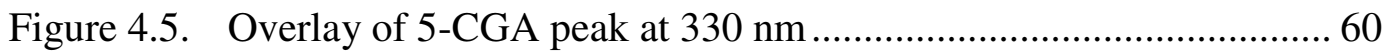

Figure 4.6. Separation of unknown peak with shoulder................................ 61 
Figure 4.7. Graph of caffeine peak area (mAU) vs. concentration $(\mathrm{mg} / \mathrm{mL})$ in linearity study.

Figure 4.8. Graph of 5-CGA peak area $(\mathrm{mAU})$ vs. concentration $(\mathrm{mg} / \mathrm{mL})$ in linearity study. 63

Figure 4.9. Overlay of caffeine standard at three concentrations 64

Figure 4.10. Sample chromatogram of 1:1 M ratio sodium caseinate:caffeine ... 65

Figure 4.11. Sample chromatogram of 1:1 M ratio sodium caseinate:5-CGA..... 65

Figure 4.12. Sodium caseinate matrix (sample blank) measured at $280 \mathrm{~nm}$........ 66

Figure 4.13. Sodium caseinate matrix (sample blank) measured at $330 \mathrm{~nm}$........ 66

Figure 4.14. Caffeine calibration curve of peak area vs. concentration................ 70

Figure 4.15. Concentration of caffeine detected in the supernatant of all samples prepared on Day 1 and Day 2

Figure 4.16. Least square means of caffeine concentration in supernatant of samples prepared at $25^{\circ} \mathrm{C}$ and $72^{\circ} \mathrm{C}$

Figure 4.17. Calculated average concentration of caffeine bound to sodium caseinate at $25^{\circ} \mathrm{C}$ and $72^{\circ} \mathrm{C}$ 73

Figure 4.18. 5-CGA calibration curve of peak area vs. concentration at $330 \mathrm{~nm}$ 74

Figure 4.19. Concentration of 5-CGA detected in the supernatant of all samples prepared on Day 1 and Day 2 .75

Figure 4.20. Calculated average concentration of 5-CGA bound to sodium caseinate at $25^{\circ} \mathrm{C}$ and $72^{\circ} \mathrm{C}$, each at $\mathrm{pH} 6.7$. 
Figure 4.21. Sodium caseinate solution before addition of green coffee extract and after addition of green coffee extract 79

Figure 4.22. Sample of sodium caseinate bound with green coffee extract after isoelectric precipitation at $\mathrm{pH} 4.6$ and $30^{\circ} \mathrm{C}$. 79

Figure 4.23. Casein pellets after centrifugation 80

Figure 4.24. Concentration of caffeine detected in the supernatant of green coffee extract with sodium caseinate samples.

Figure 4.25. Concentration of 5-CGA detected in the supernatant of green coffee extract with sodium caseinate samples. 82

Figure 4.26. Calculated average concentration of caffeine and total CGA bound to sodium caseinate from green coffee extract at optimal treatment conditions $\mathrm{pH} 6.7 \& 72^{\circ} \mathrm{C}$.

Figure 4.27. Caffeine standard (1:16 dilution) measured at $280 \mathrm{~nm}$.................. 83

Figure 4.28. CGA standard (1:16 dilution) measured at $330 \mathrm{~nm}$ 83

Figure 4.29. Sample chromatogram of 1:1 M sodium caseinate:caffeine sample 84

Figure 4.30. Sample chromatogram of 1:1 M sodium caseinate:5-CGA sample. 85

Figure 4.31. Sodium caseinate and green coffee extract sample at $280 \mathrm{~nm}$ 86

Figure 4.32. Sample chromatogram of green coffee extract only measured at $330 \mathrm{~nm}$. 86

Figure 4.33. BSA standard curve absorbance at $562 \mathrm{~nm}$ vs. concentration 87 
Figure 4.34. SDS-PAGE eletrophoretogram of sodium caseinate with

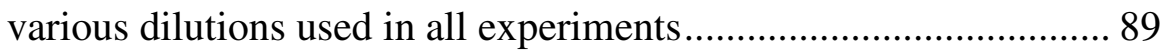




\subsection{INTRODUCTION}

The major bioactive compounds of coffee have been studied extensively, with the first isolation of caffeine from green coffee beans performed in 1820 by the German chemist, Friedlieb Ferdinand Rung. Caffeine has been linked to reducing the risk of many metabolic diseases, including diabetes and certain cancers. In addition to caffeine, coffee is an abundant source of phenolic compounds, collectively known as chlorogenic acids (CGA) that have also been shown to impart many crucial health benefits. However, light and oxygen sensitivities of phenolic compounds, or antioxidants, makes transporting these compounds to be incorporated into different food matrixes a technical challenge. Caseins in milk have been reported to be excellent delivery systems via nanoencapsulation because of their high tendency to aggregate into self-associated micelles, or by hydrophobic binding due to its unique amphipathic structure. The associative properties of $\alpha_{\mathrm{s1}^{1}}, \alpha_{\mathrm{s} 2^{-}}$, and $\beta$-caseins via hydrophobic binding can be attributed mainly to the effect of temperature.

Caseins have been specifically shown in previous studies to have excellent binding capacity with a wide variety of bioactive compounds including antioxidants, but literature on their binding capacity with caffeine is limited. The work herein attempts to elucidate the mechanism by which caffeine and CGA bind to casein micelles. First, a procedure will be established to bind these compounds to sodium caseinate. Using this procedure, we can then investigate what effects of $\mathrm{pH}$ and temperature are on the binding capacity of sodium 
caseinate with these bioactive compounds. Secondly, determining the effects of $\mathrm{pH}$ and temperature will allow for speculation on sodium caseinate's mechanism of binding. Heat treatment on caseins can lead to denaturation of the proteins, structural rearrangement, and other conformational changes within the protein which may increase the exposure and availability of hydrophobic sites for interaction with other compounds. Steric interactions and repulsive forces are mainly responsible for maintaining the structure of the micelle and preventing aggregation of the micelles. However, these interactions may also affect casein's binding capacity with other compounds through electrostatic interactions resulting from changes in the ionic strength of the chemical environment.

An analytical method to quantify these compounds by reversed phase HPLC (RP-HPLC) will be developed and validated in an effort to accurately characterize and quantify these compounds as well. 


\subsection{LITERATURE REVIEW}

\subsection{Milk proteins}

The proteins in milk provide an extremely rich source of nutrition by acting as effective binding agents and delivery systems for sensitive bioactive compounds. Milk proteins are also extremely versatile due to its various functional, physiochemical, and structural properties. Briefly, a summary of the properties of milk proteins, with specific emphasis on caseins, is provided in the following sections.

\subsubsection{Overview of milk proteins}

Milk is a fluid secreted by all female mammalian species and is the primary source of meeting the nutritional needs for the neonate. Over time, milk proteins have been remarkable at adapting to their evolutionary task of delivering vital nutrients, the most important of which are amino acids: the building blocks of proteins, in addition to calcium and phosphates. As a dietary item, milk has a pleasant taste, flavor, and mouth-feel. In addition, milk is the single most complete food available. It comes as no surprise then, that the individual components in milk have been an attractive area of research, both industrially and academically.

The composition of milk varies across species due to the unique nutritional and physiological requirements necessitated by that species (Table 2.1). Bovine milk contains approximately $3.4 \%$ protein; of which approximately $80 \%$ are caseins, and the remaining $20 \%$ are whey proteins, also known as serum proteins (Fox \& McSweeney, 1998). The major role of caseins is to transport 
calcium, essential amino acids, and antibodies, among other vital micronutrients, from the mother to the neonate. Therefore, caseins not only possess an important biological function, they are also a highly sought after functional food. Caseins are especially rich in essential amino acids; specifically $\alpha_{\mathrm{s} 2}$-caseins are rich in lysine, which plant based proteins are deficient.

Table 2.1. Composition \% of milks of some species (Fox, 2003)

\begin{tabular}{lccccc}
\hline Species & Total Solids & Fat & Protein & Lactose & Ash \\
\hline Human & 12.2 & 3.8 & 1.0 & 7.0 & 0.2 \\
Cow & 12.7 & 3.7 & 3.4 & 4.8 & 0.7 \\
Goat & 12.3 & 4.5 & 2.9 & 4.1 & 0.8 \\
Sheep & 19.3 & 7.4 & 4.5 & 4.8 & 1.0 \\
Pig & 18.8 & 6.8 & 4.8 & 5.5 & -- \\
Horse & 11.2 & 1.9 & 2.5 & 6.2 & 0.5 \\
Donkey & 11.7 & 1.4 & 2.0 & 7.4 & 0.5 \\
Reindeer & 33.1 & 16.9 & 11.5 & 2.8 & -- \\
Domestic rabbit & 32.8 & 18.3 & 11.9 & 2.1 & 1.8 \\
Bison & 14.6 & 3.5 & 4.5 & 5.1 & 0.8 \\
Indian elephant & 31.9 & 11.6 & 4.9 & 4.7 & 0.7 \\
Polar bear & 47.6 & 33.1 & 10.9 & 0.3 & 1.4 \\
Grey seal & 67.7 & 53.1 & 11.2 & 0.7 & - \\
\hline
\end{tabular}

\subsubsection{Functional milk proteins}

The principal functional food proteins derived from milk are caseins and whey proteins. The relative ease with which purified milk proteins can be obtained has caused an increase in market consumption as well as research into characterizing the properties of these proteins. Full characterization is required in order to understand and exploit the extensive applications of the functionalities of milk proteins. Because of its unique surface activities, caseins are the functional protein of choice for many food applications including their use as foaming agents, thickening agents, and for their stabilizing properties. Caseins also possess 
a high water binding capacity, which is a desirable functional property for use in various foods, such as baked products and cheese products. Prior to their use in foods, caseins have historically been used for non-food applications such as adhesives for wood, plastics, paper coating and leather and synthetic fiber finishing. Caseins are composed of a hydrophobic and a hydrophilic region, making them distinctly amphipathic. Consequently, caseins readily absorb at airwater and oil-water interfaces, giving them exceptional emulsifying properties (Fox \& McSweeney, 1998). Furthermore, caseins can be obtained by other isolation and purification methods from skim milk to result in acid casein or rennet casein, by the addition of acid or the enzyme chymosin (also known as rennet), respectively. Caseins can further be processed and dried into caseinates with the addition of sodium hydroxide to yield sodium caseinate, and calcium hydroxide to yield calcium caseinate. The difference in the composition of each type of casein can be seen in Table 2.2.

Table 2.2. Composition of casein and caseinates (Southward, 1985)

\begin{tabular}{lcccc}
\hline Component & Acid casein & $\begin{array}{c}\text { Rennet } \\
\text { casein }\end{array}$ & $\begin{array}{c}\text { Sodium } \\
\text { caseinate }\end{array}$ & $\begin{array}{c}\text { Calcium } \\
\text { caseinate }\end{array}$ \\
\hline Moisture (\%) & 11.4 & 11.4 & 3.8 & 3.8 \\
Protein (\%) & 85.4 & 79.9 & 91.4 & 91.2 \\
Ash (\%) & 1.8 & 7.8 & 3.6 & 3.8 \\
Lactose (\%) & 0.1 & 0.1 & 0.1 & 0.1 \\
Fat (\%) & 1.3 & 0.8 & 1.1 & 1.1 \\
Sodium (\%) & $<0.1$ & $<0.1$ & $1.2-1.4$ & $<0.1$ \\
Calcium (\%) & $4.6-5.4$ & $7.3-7.7$ & $6.5-6.9$ & $6.8-7.0$ \\
pH & $4.3-4.6$ & $6.5-6.7$ & $6.5-6.9$ & $6.8-7.0$ \\
Solubility in & 0 & 0 & 100 & $90-98$ \\
water (\%) & 0 & & & \\
\hline
\end{tabular}




\subsubsection{Whey proteins}

About $20-25 \%$ of the proteins in milk are whey proteins, also known as serum proteins. Whey proteins remain soluble at $\mathrm{pH} 4.6$, the isoelectric point of caseins. The major fractions of whey are $\alpha$-lactalbumin and $\beta$-lactoglobulin, representing approximately $20 \%$ and $50 \%$ of total bovine whey protein, respectively (Fox \& McSweeney, 1998). A unique characteristic of $\alpha$-lactalbumin is its biological role in the synthesis of lactose, interacting with UDP-galactosyl transferase to produce lactose from galactose (Brew, 2003). Other fractions of whey include immunoglobulins (Hurley, 2003), proteose-peptones (Rowland, 1938) and lactoferrin, among other minor milk proteins that also play important biological roles (Lönnerdal, 2003).

Whey proteins are used in a variety of food applications due to their unique functionality within a food system which include stabilization, gelation, and emulsification. Like caseins, whey proteins can be prepared from skim milk by using acid to yield acid whey, or by rennet to yield rennet (or sweet) whey. Further processing of whey by ultrafiltration, diafiltration or adsorption chromatography to remove lactose and salts results in the production of whey protein concentrates (WPC) containing 30-80\% protein, and whey protein isolates (WPI) containing about $95 \%$ protein (Fox \& McSweeney, 1998). Due to the ability to obtain such high-quality proteins, WPC and WPI are extensively used in the food industry for products such as muscle building and recovery protein drinks, in foods designed for weight management, and in prepared foods that provides high protein contents for extended satiety. 


\subsubsection{Milk proteins as delivery systems for bioactives}

Recently, interest in developing functional food systems that can also act as effective carriers and delivery systems of various bioactive molecules has gained much interest. Milk proteins contain chemical and structural versatility that make them ideal candidates for delivery of various bioactive molecules (Benshitrit et al., 2012). The ease with which milk proteins can be obtained by relatively inexpensive measures makes them a suitable target for use as a delivery system on an industrial basis. In addition, milk proteins are natural and are Generally Recognized As Safe (GRAS) for use in the food and pharmaceutical industry. Their unique surface properties allow them to deliver hydrophobic or hydrophilic bioactive molecules. They are able to form complexes with macromolecules and have innate protective qualities vital to delivering and improving the bioavailability of molecules prone to oxidation. Furthermore, milk proteins undergo extensive self-assembly and co-assembly; this causes the formation of micelles in caseins. Natural self-assembly is the basis for nanoencapsulation of nutraceuticals (Semo et al., 2007) thus harnessing this property of milk proteins can prove instrumental for both the food and pharmaceutical industry.

\subsection{Caseins}

\subsubsection{Chemistry of caseins}

About $95 \%$ of the casein in milk exists as colloidal molecules known as micelles that have a high tendency to aggregate; making them a very suitable vehicle for the delivery of bioactive molecules. Aggregation into large particles 
that precipitate readily from solution occur at temperatures between $30-35^{\circ} \mathrm{C}$ in acidic conditions. Casein micelles are responsible for the white color of milk due largely to their ability to scatter light. This white color is lost if the casein micelle structure is disrupted by removal of the inorganic constituents in casein, collectively known as colloidal calcium phosphate (CCP). Removal of the CCP results in a very different system from that of the normal milk system, namely the dissociation of the casein micelle structure.

The $\mathrm{pH}$ of milk is approximately 6.7 , at this $\mathrm{pH}$ casein micelles have a net negative charge and exists as a colloidal dispersion. Caseins are the insoluble proteins obtained by isoelectric precipitation of milk at $\mathrm{pH}$ 4.6. Casein can be obtained from milk by isoelectric precipitation or enzymatic (rennet) coagulation. This occurs after separation of the cream layer using centrifugation to produce milkfat products, such as butter and the phospholipid-rich milk fat globular membrane (MFGM). In isoelectric precipitation, the $\mathrm{pH}$ of skim milk is lowered from $\mathrm{pH} 6.7$ to $\mathrm{pH} 4.6$ at $20{ }^{\circ} \mathrm{C}$ to obtain a casein pellet after centrifugation. In the case of rennet coagulation, there is no change in the $\mathrm{pH}$ of milk, rather the enzyme rennet, also known as chymosin, is added. Chymosin cleaves a specific peptide bond in the amino acid sequence of $\kappa$-casein, $\mathrm{Phe}_{105}-\mathrm{Met}_{106}$, to result in two fragments known as glycomarcopeptide, and para-к-casein. This cleavage has a specific consequence, causing $\kappa$-casein to lose its ability to stabilize the casein micelle, resulting in an increase in surface hydrophobicity and consequently the formation of a three-dimensional gel network or clot, in the 
presence of calcium ions. This important reaction is essential in the cheesemaking process.

In contrast to globular whey proteins, which are relatively hydrophilic, caseins have high surface hydrophobicity owing to the exposure of their hydrophobic residues and relative lack of secondary and tertiary structures. High levels of proline are responsible for the lack of $\alpha$ - and $\beta$-structures (Fox \& McSweeney, 1998), necessary for formation of secondary and tertiary structures. As noted before, milk proteins are unique in their ability to bind and deliver hydrophobic or hydrophilic molecules. This notable feature can be explained by the uneven distribution of the hydrophobic and hydrophilic residues in the amino acid sequence, which gives caseins a distinctly amphipathic structure (Swaisgood, 2003). Milk proteins can bind small hydrophobic molecules by means of several mechanisms, including: hydrophobic interactions, van der Waals attraction and hydrogen bonds (Livney, 2010).

\subsubsection{Physiochemical properties of the fractions of casein}

The composition of milk is well known and its individual constituents are well characterized. As such, it has been known that caseins are composed of four individual fractions: $\alpha_{\mathrm{s} 1^{-}}, \alpha_{\mathrm{s} 2^{-}}, \beta-$, and $\kappa$-caseins. $\kappa$-caseins play an extremely important physiological role in maintaining the integrity of the micelle structure by stabilizing the caseins in the presence of calcium ions in milk. Under a hypothesized model proposed by Holt (1994), $\kappa$-casein forms a "hairy layer" on the surface of micelles and is responsible for stabilizing the micelles sterically and electrostatically from aggregation. If this "hairy layer" is removed, via specific 
hydrolysis or collapsed by ethanol, the colloidal stability of the casein micelle is compromised and results in precipitation of the casein (Holt and Horne, 1996). $\kappa$-caseins are the least sensitive to calcium salt and remain soluble under all conditions which would precipitate the other casein fractions. $\alpha_{\mathrm{s} 2}$-caseins are the most hydrophilic and most calcium sensitive of all the caseins, they precipitate at a $\mathrm{Ca}^{2+}$ concentration of less than $2 \mathrm{mM} . \alpha_{\mathrm{s} 1}$-caseins are slightly less sensitive to the presence of calcium salt, precipitating in the range of $3-8 \mathrm{mM} \mathrm{Ca}^{2+}$. $\beta$-caseins are the most hydrophobic fraction of the caseins and less sensitive yet to calcium salt, precipitating at a range of $8-15 \mathrm{mM} \mathrm{Ca}^{2+}$ at $37{ }^{\circ} \mathrm{C}$ (Swaisgood, 2003). Of all the casein fractions, $\beta$-casein is the most temperature sensitive. Monomers predominate in $\beta$-casein at low temperatures $\left(<10-15^{\circ} \mathrm{C}\right)$ but associate via hydrophobic bonding into micelles at increased temperatures (O'Connell, 2003). $\beta$-casein associates into soap-like micelles with a hydrophobic core and hydrophilic surface with increases in temperature, indicating that $\beta$-casein undergoes a temperature-induced micellization process. Being temperature dependent, this micellization process can be explained predominantly by hydrophobic bonding as the principal associative force, with electrostatic and steric interactions being responsible for maintaining the stability of the micelle and preventing the micelle from aggregating (O'Connell, 2003).

Based on primary structures, $\alpha_{\mathrm{s} 1^{-}}$and $\alpha_{\mathrm{s} 2}$-caseins are both suggested to be composed of a negatively charged N-terminal hydrophilic domain and a positively charged C-terminal hydrophobic domain. Interactions of the hydrophobic domains are likely to be responsible for the associative nature of the protein via 
hydrophobic interactions. Consequently, the associative properties of both protein fractions are highly dependent on the ionic strength and temperature of its environment. It is important to note that association of these proteins may not only be due to hydrophobic interactions exclusively, as dissociation of the protein does not occur at low temperatures, as in the case of $\beta$-casein (Swaisgood, 2003). All casein fractions share in common a distinctly amphipathic structure which confers excellent surface properties.

\subsubsection{Structural properties of casein}

Many hypothesized models of the structure of casein have been developed, though a consensus within the scientific community regarding the presence or absence of secondary or tertiary structures of the casein micelle remains yet to be achieved. In an effort to validate a hypothesized model of the casein micelle, characterization of caseins using advanced imaging techniques have been at the forefront of native casein micelle research. These imagining techniques include, but are not limited to: confocal microscopy (CM), conventional electron microscopy, scanning electron microscopy (SEM), atomic force microscopy (AFM), cryo-transmission electron microscopy (cryo-TEM) and tomographic reconstruction models. Through the combined use of these techniques and years of research resulting in a wealth of new insights into the internal architecture of the casein micelle, certain structural and dynamic properties of the casein micelle can now be visualized and confirmed unequivocally. Work by Harte and coworkers (2011) demonstrated through the use of cryo-TEM that the interior of the casein micelle not only exhibited proteins 
in a random arrangement, but that it also contained water-filled cavities and channels, as well as several hundred high-density nanoclusters (Figure 2.1). The presence of water-filled channels is not surprising, given that micelles are highly hydrated and bind approximately $2.0 \mathrm{~g} \mathrm{H}_{2} \mathrm{O} / \mathrm{g}$ protein (Fox, 2003).
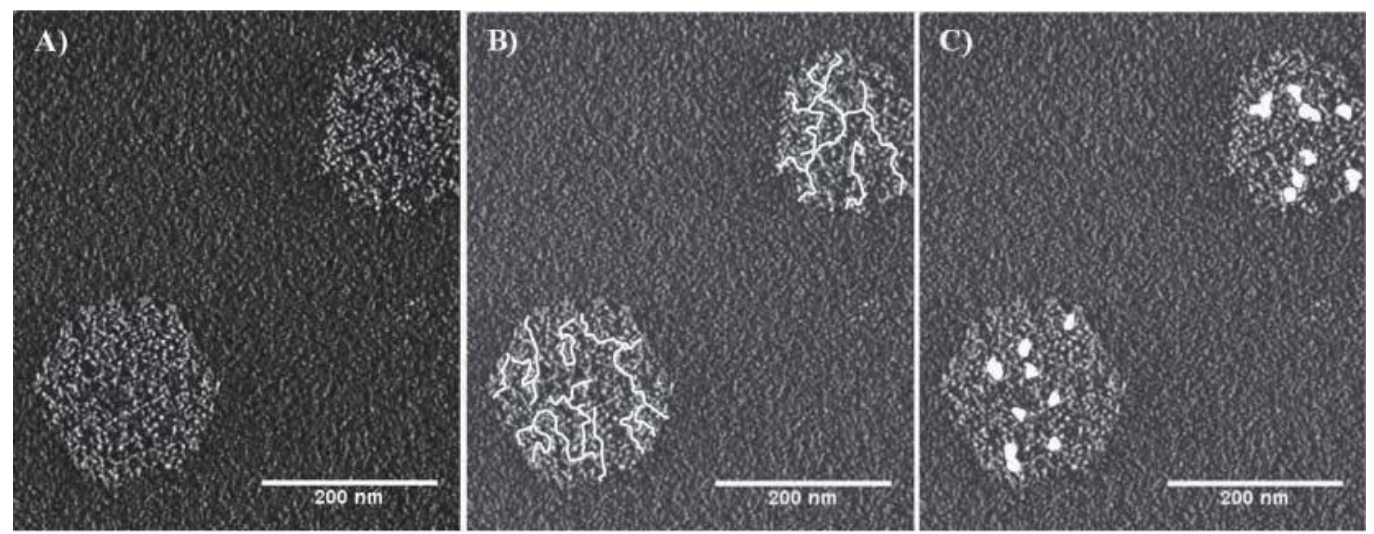

Figure 2.1. Cryo-TEM tomograms of casein micelles (Trejo et al., 2011) A) Slabs (4.74 $\mathrm{nm})$ through the centers of 2 micelles from the cryo-TEM tomograms; $B$ ) highlighting of the internal channels in the native casein micelles in the same image; $C$ ) highlighting of the internal cavities in the native casein micelles in the same image. Scale bar $=200 \mathrm{~nm}$.

\subsection{Caseinates}

\subsubsection{Preparation of sodium caseinate}

Several types of commercial caseinates are obtained from the resolubilization of the casein pellet remaining after isoelectric precipitation. Caseinates are produced by the addition of one of several dilute alkalis to adjust the $\mathrm{pH}$ of casein to $6.5-7.0$ using sodium, potassium, ammonium, or calcium. The resulting homogenous solution is then spray dried to produce a caseinate powder (Fox \& McSweeney, 1998). Sodium caseinate is the most common manufactured caseinate due to its high solubility in water and is therefore used in a wide variety of applications. Sodium caseinate has been exploited in many studies on its 
binding and harnessing ability for the preparation of nano-vehicles for bioactive molecules because of its excellent solubility properties (Livney, 2010).

\subsubsection{Sodium caseinate as a delivery system}

Sodium caseinate has been established as an excellent vehicle for the transport of bioactive molecules and in the use as a delivery system through many previous studies. One such study by Singh and coworkers (2009) investigated sodium caseinate's ability to bind to iron. Iron is an essential mineral, obtained only through diet, which aids in the production of hemoglobin present in red blood cells and is responsible for transporting oxygen and distributing it to cells throughout the body for metabolism. A lack of iron results in iron deficiency and anemia. The iron content in milk is approximately $0.2-0.5 \mathrm{mg} / \mathrm{iron} / \mathrm{L}$ (Sugiarto, 2009) and is considered too low as the recommended daily allowance (RDA) according to the Center for Disease Control and Prevention (CDC) is about 8 $\mathrm{mg} /$ day in most adults; therefore fortification of milk and dairy products is necessary. Sodium caseinate and whey protein isolate (WPI) were used to determine the binding affinity of iron to those milk proteins. Their results showed that sodium caseinate contained more binding sites $(n=14)$ than WPI $(n=8)$ for binding iron, likely due to the presence of clusters of phosphoserine residues present in casein that are known to bind well to divalent cations (Sugiarto, 2009). The number of phosphoserine residues present in casein is different between the individual fractions of casein. $\alpha_{\mathrm{s} 1}$-casein contains the most residues and $\kappa$-casein containing the least, suggesting binding of iron occurs between the individual fractions, rather than the casein micelle as a whole. The discrepancy in binding 
affinities between sodium caseinate and whey protein may be explained by the difference in their chemical structure. Whey proteins are large globular proteins with a relatively compact, rigid structure whereas caseins possess a more open and flexible structure owing to the relative lack of secondary and tertiary structures, which facilitates the binding of various bioactive molecules, including metal ions such as iron. It is important to note that binding affinity is a function of environmental conditions, such as $\mathrm{pH}$ and temperature; suggesting binding may be due to electrostatic, hydrophobic, or a combinatory effect of both interactions.

Curcumin is a polyphenolic compound found in the spice turmeric, which has been implicated in decreasing the risk of Alzheimer's disease in India, a country known to use and consume large amounts of turmeric in their traditional foods (Govindarajan, 1980). Curcumin has also been shown to possess anticancer, anti-microbial, and anti-inflammatory properties in addition to its antioxidant activity (Maheshwari et al., 2006). However, the low solubility of curcumin in aqueous solutions remains a limitation in effectively delivering the bioactive compound to be metabolized. Binding curcumin to protein complexes, particularly water soluble milk proteins, and delivering the compound to the site where it can be metabolized may possibly mitigate this problem. Corredig and coworkers (2012) have shown that curcumin binds to nonpolar regions of the casein micelle, indicating that binding occurs mainly due to hydrophobic interactions.

Sodium caseinate has also been shown to be effective carriers of many other bioactive compounds of various chemical properties, such as soy 
phospholipids (Istarova, 2005), vitamin $\mathrm{D}_{2}$ (Semo et al., 2007), omega-3

polyunsaturated fatty acids (Zimet et al., 2011), and gum arabic (Ye, 2012).

\subsection{Coffee}

\subsubsection{History and background}

Coffee is a popular beverage consumed throughout the world from as far back as the tenth century, though it may have been cultivated in indigenous Ethiopia as early as 575 A.D. (Dews, 1984). According to historical records, the first reliable reference of a caffeine-containing beverage described in a Chinese dictionary is tea around 350 A.D., long before the first mention of coffee. Coffee was consumed as a hot beverage around 1000 A.D. It has a pleasant taste, aroma and is consumed both for its flavor and stimulating effects of caffeine alike. It is a versatile food; its grounds can be incorporated into other foods, it can be sweetened and used in baked goods and of course, it can be brewed and consumed as a beverage. The majority of coffee beverage consumption is from the Coffea arabica (Arabica) and Coffea canephora (Robusta) species. Arabica coffee is considered to have a superior taste to Robusta coffee and is why it represents about $70 \%$ of the world market (Lean, 2012) and reaches higher prices in the international market (Gielissen \& Graafland, 2009). Robusta beans on the other hand, are often used in instant coffees.

Coffee consumption rates rose from $3.5 \mathrm{~kg} /$ person/year to 4.4

$\mathrm{kg} /$ person/year in the United States from 1995 to 2007, and from 3.3 $\mathrm{kg} /$ person/year to $4.1 \mathrm{~kg} /$ person/year in Europe (World Resources Institute, 2011). The desirable effect of caffeine is undoubtedly one of the major contributors to 
this increase in coffee consumption. Coffee also contains many micronutrients, such as magnesium, potassium, niacin and vitamin E, all of which could contribute to the overall health benefits of coffee consumption (Higdon \& Frei, 2006). However, coffee is composed of over a thousand different compounds that contribute both to its taste and beneficial attributes, yet many of these compounds remain yet to be characterized.

\subsubsection{Composition of the coffee fruit}

The coffee fruit is composed of several layers that each have a distinct function in protecting the cherry at the center of the bean and are depicted in Figure 2.2. The coffee bean is composed of three distinct layers: a smooth but tough outer skin layer known as the pericarp, followed by a fibrous layer of sweet pulp, called the outer mesocarp, and finally a thin yellowish layer known as the endocarp or parchment. Between the mesocarp and endocarp layers lies a translucent, colorless and viscous layer of mucilage, also known as the pectin layer. The coffee cherry, or endosperm, is covered by a layer known as the silverskin (Belitz et al., 2009; Berbert et al., 2001). Upon ripening of the fruit, the pericarp changes colors ranging from yellow to red, depending on the specific genotype. The amount of caffeine found in the pericarp is approximately two to ten times lower than that of the amount found in the seed. This is primarily because caffeine is only synthesized in the earlier developmental stages of the seed and thus is not continually synthesized during fruit maturation (Esquivel \& Jimenez, 2012). 


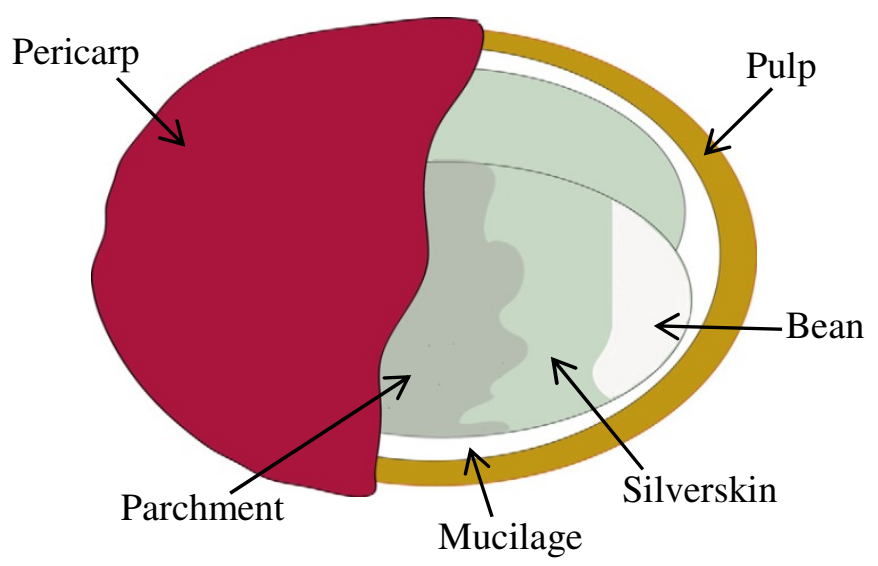

Figure 2.2. Layers in a coffee fruit (Esquivel \& Jimenez, 2012).

\subsubsection{Processing \& effects of roasting on coffee beans}

Green coffee beans can be processed by one of two methods, via dry or wet processing. Dry processing involves drying the coffee fruit in the sun before being mechanically hulled, removing the dried husks containing the skin, pulp, mucilage and parchment layers in addition to much of the silverskin in a single step. Wet processing is a much more involved process which includes separation of the ripe and unripe berries in water based on their density. The outer layers of the fruits are removed by pressing the fruit through a screen with water. The middle layers of the fruit are removed next through a fermentation step which hydrolyzes the mucilage layer using enzymes from within the coffee tissues as well as from microorganisms found on the skin of the fruit (Belitz et al, 2009; Vaast et al., 2006). The final hulling step removes the parchment layer from the beans after drying. An additional polishing step is optional to remove the silverskin layer. Though the multistep wet process method takes significantly more time and energy, it has been shown that this method yields higher contents 
of CGA and trigonelline, and a lower content of sucrose in comparison to the dry method (Duarte et al., 2010).

Sensory attributes of a coffee beverage such as flavor, aroma and body, are developed during the roasting process and due largely in part by the CGA content in the beans, which gives coffee its characteristic and desirable properties. Therefore, the amount of CGA is a large determinant of coffee cup quality. During processing and roasting, CGAs may be degraded into low molecular weight compounds through hydrolysis, thus dark roast coffee tends to contain a lower amount of CGAs compared to its unroasted or light roast counterpart (Farah \& Donangelo, 2006). The sensory profile of roasted coffee is a result of Maillard reactions, a complex series of reactions that take place at high temperatures and occur in the presence of amino acids and reducing sugars. Because CGA contributes to the antioxidative properties of roasted coffee as well, it makes sense that higher temperature of roasting results in a reduction of total CGA content (Moon et al., 2009). Green coffee beans subjected to severe roasting conditions can result in up to $95 \%$ loss of CGA (Trugo \& Macrae, 1984).

However, studies have also shown that roasted coffee extract have antibacterial activity against several strains of enterobacteria (Almeida et al., 2006) as well as microorganisms such as Staphylococcus aureus and Streptococcus mutans (Daglia et al., 2007; Dacarro et al., 1995). Further, consumption of roasted coffee beverages may increase populations of Bifidiobacterium spp., the beneficial strain of bacteria found in probiotics, which 
indicates that coffee consumption may possibly have some prebiotic effects as well (Jaquet et al, 2009).

\subsubsection{Coffee bioactives}

Coffee has a complex chemical spectrum containing over a thousand compounds, many of which still remain unidentified and thus difficult to assess the possible health benefits or adverse effects upon consumption. Of the characterized compounds, coffee contains varying levels of caffeine depending on the type of bean, with Robusta beans containing significantly more caffeine than Arabica beans. Many consumers of coffee do so because of the stimulating effects and increased alertness imparted by caffeine, which accounts for 1 to $4 \%$ of the bean on dry basis, and has a half-life of about 5 hours in most adults (Lean, 2012). Green coffee beans also consist of many phenolic compounds which account for up to $14 \%$ on dry matter basis (Esquivel \& Jimenez, 2012). These phenolic compounds are esters of trans-cinnamic acids and quinic acids. Esterification can occur at positions 3,4 , or 5 and results in several isomers of CGA. Other phenolic compounds found in green coffee beans are caffeic, ferulic and dimethoxycinnamic acids.

Other bioactive compounds that have been reported in green coffee beans are cafestol and kahweol (Figure 2.3). These diterpenes have been implicated in both positive and negative health roles. They have been shown to increase total serum cholesterol levels (Farah \& Donangelo, 2006) and production of low density lipoprotein cholesterol (LDL) (Higdon \& Frei, 2006). However, kahweol has also been identified as having potential anti-inflammatory effects (Cardenas 
et al., 2011). In addition, cafestol shows anti-oxidative properties, based on decreased DNA damage to cells treated with the diterpene (Lee \& Jeong, 2007). Diterpenes in coffee may also promote the elimination of carcinogens. However, as with all constituents of coffee, the concentration of these compounds varies greatly depending on the type of bean, roasting procedures and brewing method. Interestingly, these diterpenes have only been found in unfiltered coffee, such as coffee obtained from boiling or by using a French Press.

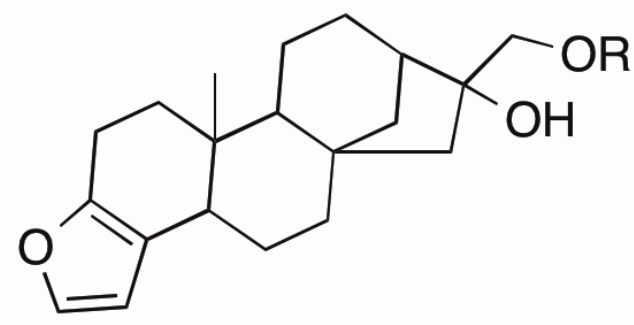

Cafestol

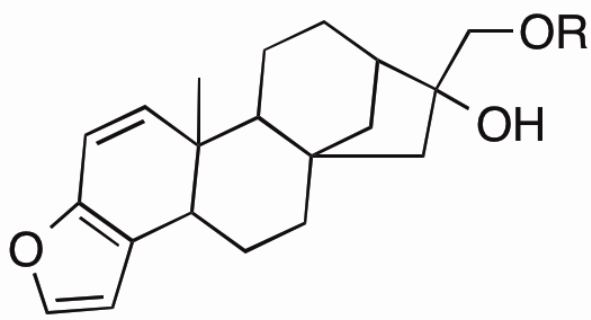

Kahweol

Figure 2.3. Chemical structures of cafestol and kahweol (Higdon \& Frei, 2006) $R=H:$ free diterpene; $R=$ fatty acid: diterpene ester

\subsubsection{Caffeine}

The chemical name for caffeine is 1, 3, 7-trimethylxanthine (Figure 2.4) and is a purine alkaloid that is naturally occurring in coffee beans. Caffeine is essentially completely metabolized by the liver into its primary metabolite, paraxanthine, and is catabolized to form xanthine and theobromine. Physiological effects of caffeine include central nervous system stimulation, increased awareness and metabolic rate, increased concentration, and acute elevation of blood pressure, in addition to diuresis (Higdon \& Frei, 2006). These effects can be felt relatively soon after ingestion. Caffeine is absorbed rapidly and almost 
completely in the stomach and small intestine and distributed to all tissues, including the brain, which is likely why stimulation of the central nervous system occurs. Additional evidence on the stimulatory effects of caffeine is due to its antagonism of $A_{1}$ and $A_{2 A}$ subtypes of the adenosine receptors (James, 2004). Adenosine (Figure 2.4) is a constituent of adenosine triphosphate (ATP) and acts as an intermediary in a wide range of metabolic pathways, including behavioral influences of xanthines. The effects of adenosine are opposite that of caffeine, suggesting an antagonistic relationship and resulting in effects of caffeine that are generally stimulatory. This supports the hypothesis that xanthines act behaviorally by blocking adenosine receptors to elicit locomotive enhancement (Dews, 1984).

As a natural product, caffeine has been used as a major ingredient in food products, including soft drinks, baked goods, dairy desserts, and candy, as well as an active ingredient in many pharmaceuticals. For instance, the use of caffeine in analgesics may be due to the fact that caffeine has been found to decrease the systemic elimination of acetaminophen and increase the bioavailability of aspirin (Higdon \& Frei, 2006). The wide versatility of caffeine gives impetus to research investigating its use as an ingredient in foods, as well as into its metabolism and positive or adverse effects to health upon ingestion. 
<smiles>Cn1c(=O)c2c(ncn2C)n(C)c1=O</smiles><smiles>Nc1ncnc2c1ncn2[C@@H]1O[C@H](CO)[C@H](O)[C@H]1O</smiles>

Figure 2.4. Chemical structures of caffeine and adenosine

\subsubsection{Chlorogenic acid (CGA)}

Coffee is also an abundant source of polyphenolic compounds, which commonly act as antioxidants. Structurally, polyphenols are characterized by having one or more aromatic rings with one or more hydroxyl groups attached. Phenolic compounds are secondary metabolites that are ubiquitous constituents involved in a higher plants defense mechanism against environmental stress. Conditions such as severe weather, excess ultraviolet (UV) or high visible light, and water stress can directly impact the amount of phenolic compounds produced by a plant (Farah \& Donangelo, 2006). The amounts of phenolic compounds present in coffee beans thus varies depending largely on cultivar, degree of maturation, and are attributed to a lesser extent by agricultural practices (Clifford, 1985).

Present as a family of esters formed between trans-cinnamic acids and quinic acid, the phenolic fraction of coffee is collectively known as chlorogenic acids (CGA). CGAs have been found on the surface within the cuticular wax and also in the cytoplasm of the coffee seed. CGA is a broad classification of the 
antioxidants present in coffee which also include: caffeoylquinic acids (CQA), dicaffeoylqunic acids (diCQA), feruloylqunic acids (FQA), $p$-coumaroylquinic acids ( $p$ CoQA) and mixed diesters of caffeic and ferulic acids with quinic acid, each group containing at least three isomers. The nine main isomers of CGA are: 5-CQA, 4-CQA and 3-CQA; 3,5-diCQA, 4,5-diCQA and 3,4-diCQA, 5-FQA, 4FQA and 3-FQA (Farah \& Donangelo, 2006). CGA and its isomers make up the main components of the phenolic fraction of the unroasted, green coffee beans, reaching levels of up to $14 \%$ on dry matter basis (Farah \& Donangelo, 2006). In green coffee beans, 5-CGA (Figure 2.5) alone accounts for about 56-62\% of total CGA, with 4-CGA and 3-CGA isomers accounting for up to $10 \%$ each of total CGA (Farah \& Donangelo, 2006). As such, esters of CGA are usually formed on carbon-5 of quinic acid but also on carbons- 3 and 4 , and less commonly on carbon-1. Roasting causes isomerization of CGA resulting in a substantial decrease in substitution at the carbon-5 position and an increased level of substitution at the carbon-3 and 4 positions. (Trugo \& Macrae, 1984; Leloup et al., 1995, Farah et al., 2005). The processing conditions, such as temperature, as well as brewing conditions (water temperature, water to bean ratio) also has a marked effect on the amount of CGA that is extracted from the coffee bean. 


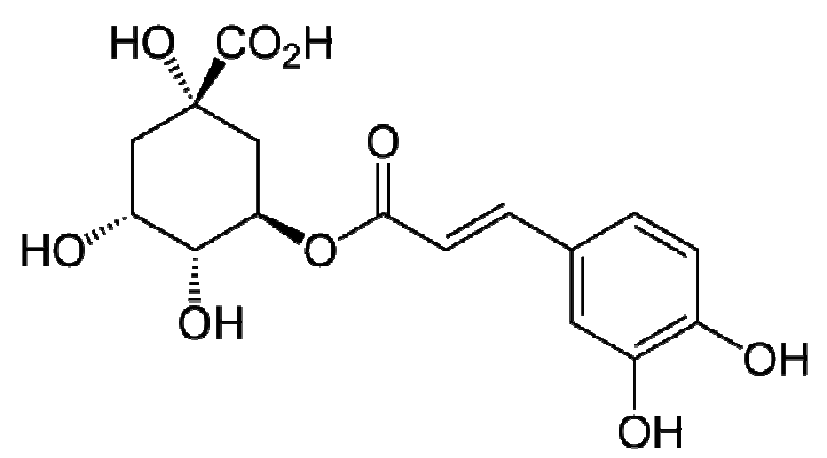

Figure 2.5. Chemical structure of 5-CGA

\subsection{Health benefits of coffee bioactives}

\subsubsection{Health benefits of caffeine}

Coffee consumption is undoubtedly made popular by the increased alertness and stimulating effects of caffeine. It is the beneficial health effects of coffee bioactive compounds that make it a functional food. Due to this functionality and the increase in awareness of health and nutrition of foods, it is imperative that these individual components be studied extensively for any beneficial as well as adverse effects upon ingestion. Caffeine has been well characterized and has been the focus of many areas of research, with epidemiological studies being at the forefront of investigating the associations between caffeine and potential health benefits in those who consume coffee. Some of the health benefits of caffeine include reduced risk of depression in women with a mean age of 63 years that regularly consume coffee (Lucas et al., 2011) as well as reduced cognitive decline in older women. Caffeine in coffee has also been observed to be inversely associated with the risk of Parkinson's disease in men in case-control studies (Hernan et al., 2002). The results from these studies failed to show a correlation between coffee consumption in women and a 
decreased risk of Parkinson's disease. These results may be attributed to the modifying effect of estrogen replacement therapy inhibiting cytochrome CYP1A2, which is also responsible for caffeine metabolism (Pollock et al., 1999).

\subsubsection{Health benefits of chlorogenic acid}

CGAs have numerous beneficial health properties due to their potent antioxidant activity, some of which include hepatoprotective, hypoglycemic and antiviral activities in addition to cell vitality (Cheng, 2011).

Epidemiological studies have suggested that the major phenolic compounds in coffee, caffeic acid (CA) and CGA, and regular coffee consumption have an inverse correlation with the incidence of Type 2 diabetes (Greenberg et al., 2005). One of the causative factors of Type 2 diabetes may be due to misfolding of human islet amyloid polypeptides (hIAPP). Huang and coworkers (2011) have investigated the inhibitory effects of caffeine, CA, and CGA on toxic aggregation of hIAPP. This study has immense implications since Type 2 diabetes constitutes $90-95 \%$ of diabetic patients worldwide (Clark et al., 1987). These studies concluded that while caffeine appeared not to have a significant inhibitory effect on the formation of lineal hIAPP fibrils, when compared to the major polyphenolic compound ( - ) -epigallocatechin 2-gallate (EGCG) found in tea, CA and CGA did have a marked inhibitory effect as detected by tioflavin-T (ThT) fluorescent dye binding studies and TEM imaging of the aggregates (Figure 2.6). The concentration of the coffee bioactive compound to hIAPP was also observed to affect the extent of inhibition. Since these studies were conducted in vitro, many physiological events such as 
metabolism of the coffee bioactives into their metabolites and varying concentrations of hIAPP present in the body, the data from these studies suggest that regular coffee consumption may inhibit hIAPP amyloid formation, thus lowering the risk of Type 2 diabetes. A significant inverse association between the risk of Type 2 diabetes and coffee consumption has also been observed in many other epidemiological studies (Salazar-Martinez et al., 2004, Rosengren et al., 2004).

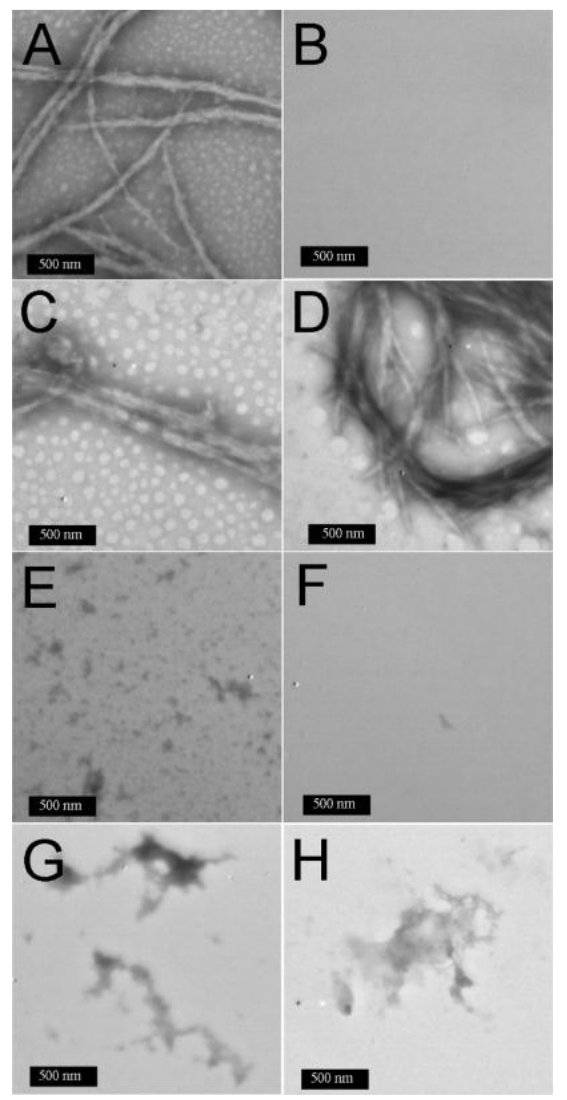

Figure 2.6. TEM images of hIAPP co-incubated with different compounds (Cheng, 2011). (A) hIAPP; (B) 1:1 mixture of hIAPP and EGCG; $(C, E, G)$ equimolar amounts of caffeine, $C A$, and $C G A ;(D, F, H) 5$-fold molar excess of caffeine, $C A$, and CGA.

Inflammation has long been associated with a plethora of cardiovascular diseases, which include atherosclerosis, cancer, obesity, and diabetes (Lai et al., 
2012). Cardiovascular disease continues to be the leading cause of death in developed countries such as the United States. Environmental factors such as diet have an immense impact on general health, one of which is modulating inflammation. Inflammation is a response triggered by noxious stimuli caused by either physical or chemical trauma. Phenolic compounds such as CGA have been shown to attenuate inflammation and confer anti-inflammatory effects (FrostMeyer \& Logomarsino, 2012). Since polyphenols are extensively metabolized in the body, consuming polyphenol-rich foods can help mitigate the effects of chronic inflammation and aid in the prevention of cardiovascular diseases. However, one main disadvantage of polyphenol consumption is their relatively low bioavailability in the body and thus remains a technological challenge. Developing functional foods that effectively carry and deliver polyphenols to the site where they can exert their antioxidant potential has gained considerable attention.

\subsection{Techniques of analysis}

Because of the large variation in the amount of bioactive compounds present in coffee beans, it is imperative to develop reliable and sensitive methods for establishing levels of these bioactive compounds. Given the discrepancy between results obtained by high resolution chromatographic methods and by less sophisticated methods, the need to continually develop and optimize sensitive methods of detection and quantification is of paramount importance. Many compounds in coffee remain uncharacterized due largely to lack of commercial standards and similarities of their chemical structures. Consequently, sensitive 
methods for proper identification and quantification are in high demand. Briefly, a summary of common methods employed in the isolation, determination and quantification of the coffee bioactive compounds, caffeine and CGA, will be discussed below. Methods of protein determination will also be reviewed.

\subsubsection{High Pressure Liquid Chromatography (HPLC)}

Separation of compounds using HPLC is achieved by exploiting differences in polarity. Typically, compounds are carried via an aqueous solution of low ionic strength and attached to a stationary phase. There are many chromatographic methods used for separating compounds including: normal phase, reversed phase, ion-exchange, size-exclusion or gel permeation, metal interaction, and affinity chromatography. In the most commonly used method, reversed phase HPLC (RP-HPLC), the stationary phase is a column composed of a matrix of polar silica gel substituted with nonpolar hydrocarbon chains (e.g. $\mathrm{C}_{8}$ or $\mathrm{C}_{18}$ alkyl chains). Compounds are carried through the column using a polar mobile phase and elution is obtained by increasing the hydrophobicity of the mobile phase. Compounds are driven by hydrophobic interactions to adsorb to the stationary phase (Swadesh et al., 1993). The compound of interest, the analyte, is then eluted based on its polarity with the most polar compounds eluting first and the less polar compounds eluting last. Nonpolar compounds have an affinity to interact with the relatively nonpolar hydrocarbon chains attached to the stationary phase and are retained longer than more polar compounds. The dimensions of a column, length and diameter, as well as the particle size of the packing material have a large effect on separation and resolution of analyte peaks and on 
equilibration time. Therefore, the analytical column often has an impact on the performance of a chromatographic method.

Chromatographic conditions may employ the use of isocratic or gradient elution. In isocratic elution, a single mobile phase remains at a fixed concentration throughout the analysis. In gradient elution, several mobile phases of various ionic strengths are mixed together by a high pressure pump and the concentration of the mobile phases varies throughout the run. Gradient elution is preferred over isocratic elution if determination of several analytes within a single sample is needed.

There are several types of detectors used in HPLC analysis, each having the ability to detect different types of molecules. UV-detectors are commonly used to detect an array of small organic molecules that possess a chromaphore, a part of their chemical structure that has the ability to absorb UV-light (e.g. conjugated double bonds or aromatic rings). RP-HPLC has also been extended for the analysis of macromolecules, such as proteins. A major limitation to UVdetectors is being able to measure only at one specified wavelength. Hence if there are several analytes in a sample that absorb at different wavelengths, using a UV-detector would mean having to inject the sample multiple times at different wavelengths to completely analyze the sample. A solution to this problem is to use a photo-diode array (PDA) detector. PDA detectors are capable of analyzing a sample at multiple wavelengths, allowing one sample with multiple analytes to be analyzed at once, saving time and money. 
Several methods exist for the simultaneous determination of purine alkaloids and polyphenols by HPLC (Samanidou et al., 2012, Ramalakshmi et al., 2011, Trugo et al., 1991). More sophisticated and sensitive methods of analysis, such as tandem mass spectroscopy, have also been employed for the determination of polyphenols in green coffee beans (Alonso-Salces et al., 2009). Caffeine in various coffee brews have also been determined by a rapid method known as direct analysis in real time (DART) ion source coupled to highresolution time-of-flight mass spectrometry (TOFMS) (Danhelova, et al., 2012). This sensitive method is not only capable of detecting and quantifying caffeine, it is also able to detect other polar compounds isolated from Arabica and Robusta coffee brews, measured in both positive and negative ion modes (Figure 2.7). Rapid and powerful methods of analysis such as DART-TOFMS may be used in the future in assessing the authenticity of coffee. Selection of the method and technique depends on the level of sensitivity needed for an analysis, and the availability of the instruments. However, analytical methods are often compared to results obtained by HPLC (Trugo et al., 1991) due to the simplicity of sample preparation, accurate results, availability and affordability of the instrument, and relatively low level of training required to complete an analysis. 


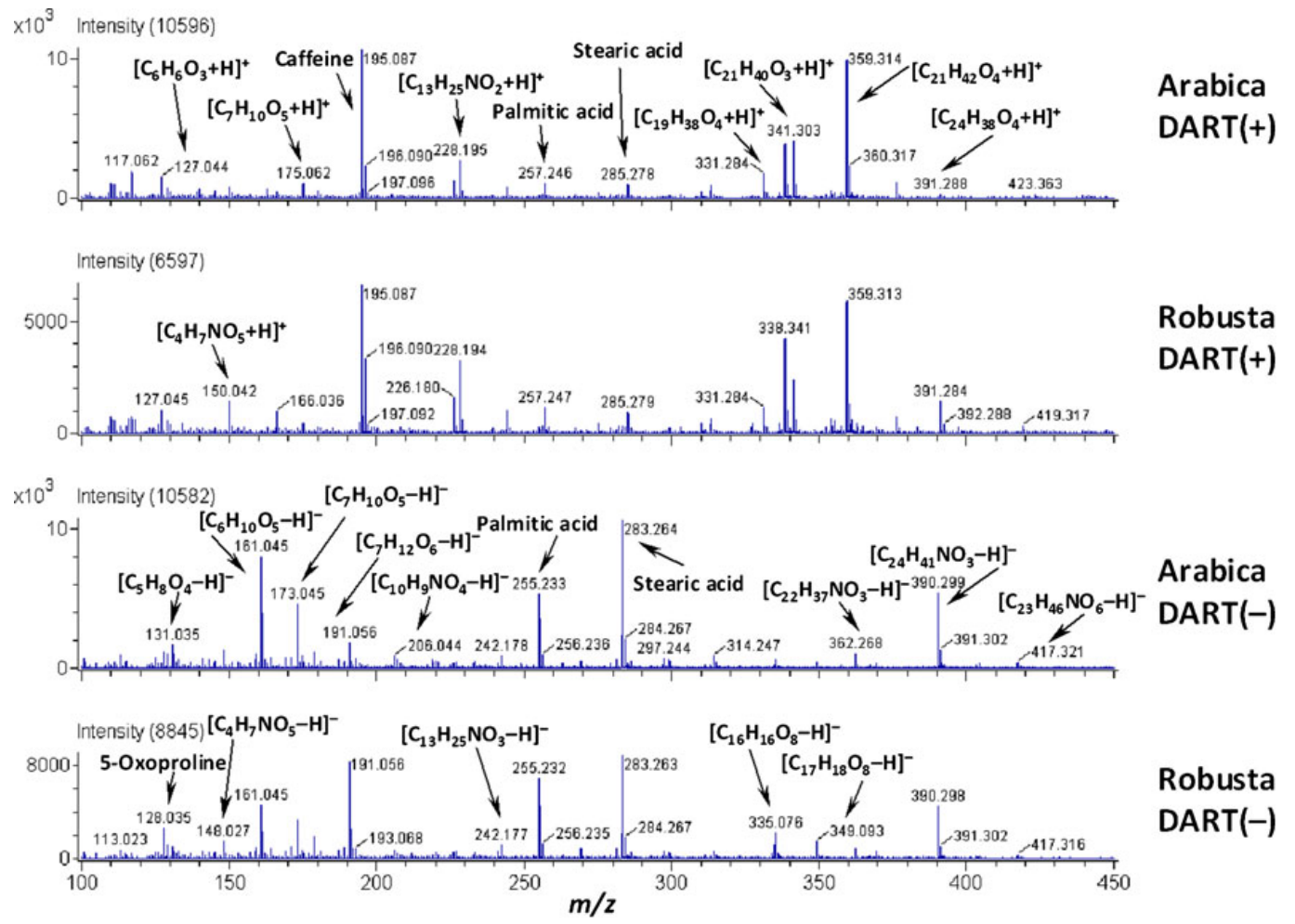

Figure 2.7. DART-TOFMS spectra (Danhelova et al., 2012). ( $\mathrm{m} / \mathrm{z}, 100-450)$ acquired in DART positive and negative ion modes of the extracts of roasted ground Arabica and Robusta coffee brew.

\subsubsection{Oxygen Radical Absorbance Capacity (ORAC) assay of antioxidants}

Antioxidants are an important class of compounds used as preservatives in foods. They are radical inhibitors that prevent the oxidation of foods by preventing unwanted radical reactions by reactive oxygen species (ROS). An excess of ROS in the body leads to cellular injury and damage to proteins, which in turn plays a role in the development of many diseases, such as diabetes, cardiovascular disease, and neurodegenerative diseases. Consumption of antioxidants from food sources is one way to decrease ROS-induced oxidative stress in the body. In order to evaluate whether a particular food is an adequate source of antioxidants, it is important to assess the antioxidant capacity of its extracts. A classical tool for measuring antioxidant capacity is the Oxygen 
Radical Absorbance Capacity (ORAC) assay. The ORAC assay is capable of measuring the antioxidant capacity of biomolecules from a variety of samples, making it an extremely versatile assay. In addition to its versatility, the assay can be performed with relative ease. An ORAC assay kit obtained from Cell Biolabs, Inc. (San Diego, CA) is a fast and reliable kit for the direct measurement of the antioxidant capacity from various food extracts. Once the appropriate reagents are prepared, sample preparation in a multi-well microtiter plate is performed by adding the sample and a fluorescein solution to each well in a 1:6 ratio and incubating at $37^{\circ} \mathrm{C}$ for 30 minutes. The fluorescein solution is a fluorescent probe which is oxidized by peroxyl radicals. A solution of free radical initiator is added to each well and mixed immediately prior to being placed in a microplate reader at $37{ }^{\circ} \mathrm{C}$ and analyzed with an excitation wavelength of $480 \mathrm{~nm}$ and emission wavelength of $520 \mathrm{~nm}$ (Figure 2.8). The free radical initiator produces the peroxyl radicals to quench the fluorescent probe over time. Therefore, the antioxidants present in the sample works to block the peroxyl radical oxidation of the fluorescent probe and the antioxidant capacity of the sample is measured by comparison with a standard curve of vitamin $\mathrm{E}$, measured in Trolox ${ }^{\mathrm{TM}}$ equivalents (TE). Another advantage of the ORAC assay is its ability to combine both inhibition time and degree of inhibition into a single quantity, the only method thus far capable of providing these results (Ramalakshmi et al., 2011). 


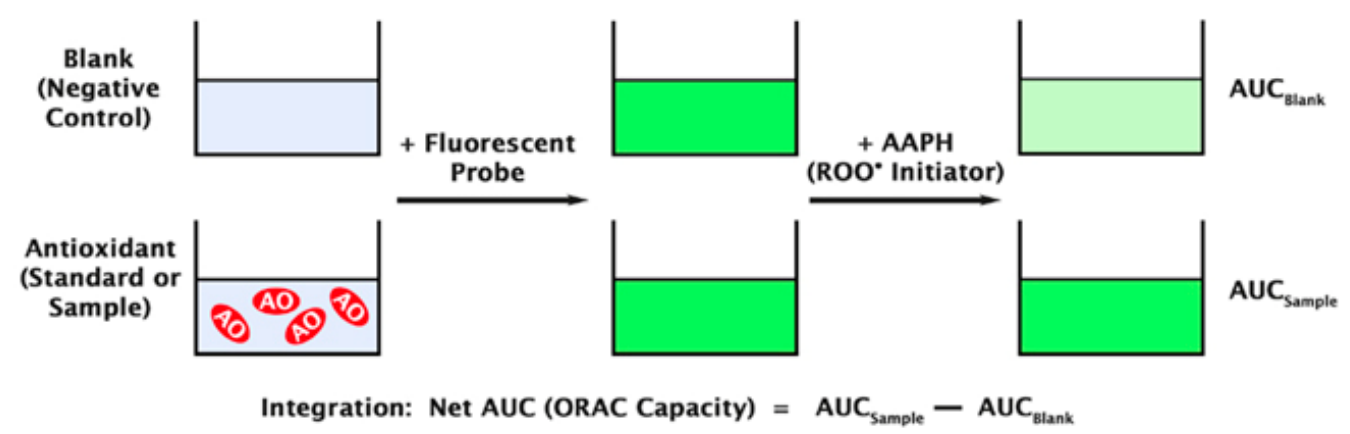

Figure 2.8. ORAC assay principle. ORAC assay of a sample is calculated by subtracting the area under the curve (AUC) of the blank from the AUC of the sample to obtain the net AUC (Cell Biolabs, Inc.).

\subsubsection{Protein analysis}

Numerous methods for the quantification of protein have been developed. The Official Methods of Analysis of AOAC International include the Kjeldahl, Dumas, and infrared spectroscopy methods (AOAC, 2000). The Kjeldahl method (AOAC, 995.04), developed in 1883 by Johann Kjeldahl, involves digestion of protein with sulfuric acid and conversion of the protein nitrogen to ammonium sulfate by oxidation with potassium permanganate, neutralization of the digest, and finally titration to obtain the percent nitrogen. A conversion factor is then used to convert percent nitrogen to percent protein. Although the Kjeldahl method for protein analysis is an AOAC method and commonly used, it can be quite time consuming and requires the use of hazardous chemicals. An alternative to the Kjeldahl method is the Dumas method (AOAC, 992.23), developed in 1831 by Jean-Baptiste Dumas. Samples are weighed into a tin capsule and placed in a combustion reactor equipped with a built in gas-chromatograph (GC). The nitrogen released by the sample is then collected and measured directly by the GC. This automated process takes considerably less time to analyze samples, and 
requires no hazardous chemicals. However, like the Kjeldahl method, the Dumas method measures total organic nitrogen and therefore does not distinguish between protein and non-protein nitrogen (NPN). Infrared spectroscopy is used to measure the absorption of radiation by peptide bonds to estimate the protein concentration in a food sample (AOAC, 997.06).

Another alternative method for the quantification of protein in a sample is the Bicinchoninic acid (BCA) method. The BCA method exploits the ability of proteins to reduce $\mathrm{Cu}(\mathrm{II})$ to $\mathrm{Cu}(\mathrm{I})$ under alkaline conditions (Smith et al., 1985). A purple-colored product is obtained by the chelation of two molecules of BCA with one cuprous ion. The color formed is proportional to the protein concentration in a sample as measured by a spectrophotometer at $562 \mathrm{~nm}$. The concentration of protein in a sample is then compared to a standard curve of known concentrations of a protein such as Bovine Serum Albumin (BSA). Therefore, the BCA assay is used as a colorimetric method for the determination and quantification of total protein in a sample. This is a simple and widely used, albeit time sensitive method of protein analysis.

\subsection{Justification for work}

Given the increase in coffee beverage consumption in addition to an increase in popularity of incorporating pure extracts of coffee bioactive compounds into a wide variety of food products, it is necessary, if not imperative to develop analytical methods using sophisticated instruments that are both fast and reliable. Rapid methods of analysis facilitates the use of these compounds in ways that may not have been previously possible, such as using milk proteins to 
bind and deliver into different food matrixes. Limited research has been done on the binding capacity of sodium caseinate with the major alkaloid in coffee, caffeine. Given the numerous health benefits conferred by caffeine and regular consumption of caffeine and antioxidant containing beverages such as tea and coffee; much attention has been attracted to projects involving the use of both these compounds. However, incorporation of these compounds has proven a technical challenge due to their susceptibility to oxidation. Binding these compounds with a protective vehicle for transport; such as casein micelles in milk proteins, may mitigate this challenge.

The objectives of this thesis are to first develop and validate an HPLC method for the accurate and simultaneous determination of caffeine and CGA in sodium caseinate samples. Next, is to establish a procedure by which caffeine and CGA can be bound to sodium caseinate and investigate the effects of $\mathrm{pH}$ and temperature on its binding capacity. And last is to, determine the conditions that result in increased binding interactions and estimate the binding capacity of sodium caseinate with these bioactive compounds and speculate on their binding mechanism. Knowledge gained from these studies could promote the use of milk proteins in even more functional ways by elucidating the mechanisms and conditions by which proteins bind with these compounds and how changes in the chemical environment can increase binding interactions. Moreover, these studies aim to promote the use of dairy proteins with the health promoting constituents of coffee and to provide a means by which accurate and reliable quantification of these compounds can be achieved. 


\subsection{MATERIALS AND METHODS}

3.1 Development of a method for the simultaneous determination of caffeine and CGA by reversed phase High Pressure Liquid Chromatography (RP-HPLC)

\subsubsection{HPLC Method optimization for the analysis of caffeine and CGA in green coffee extracts}

\subsubsection{Mobile phase gradient}

Mobile Phase A (MPA) was prepared in a 90:10:0.1 (v/v/v) of MilliQ Nanopure water/acetonitrile/formic acid. MilliQ Nanopure water (900 mL) was transferred to a suitable media bottle and $1.0 \mathrm{~mL}$ of formic acid $(97 \%$ ACS grade, Acros Organics) was added and mixed well. The solution was then filtered through a polyethersulfone (PES) membrane filter (Cat \# 431118, $0.22 \mu \mathrm{m}$, Corning Inc.) under vacuum. Acetonitrile (100 mL) (HPLC grade, Fisher Scientific) was then added to the solution and mixed well. Mobile Phase B (MPB) was prepared in a 75:25:0.1 (v/v/v) of MilliQ Nanopure water/acetonitrile/formic acid. MilliQ Nanopure water $(750 \mathrm{~mL})$ was transferred to a suitable media bottle and $1.0 \mathrm{~mL}$ of formic acid (97\% ACS grade, Acros Organics) was added and mixed well. The solution was then also filtered through a PES membrane filter (Cat \# 431118, $0.22 \mu \mathrm{m}$, Corning Inc.) under vacuum. Acetonitrile (250 mL) was then added to the solution and mixed well. The HPLC unit used (Shimadzu Prominence, CBM-20A) was equipped with an autosampler (SIL-20A), column oven (CTO-20A), and a photo-diode array (PDA) detector (SPD-M20A). The column employed was a Shimadzu $\mathrm{C}_{18}$ reversed phase (RP) column (Shimadzu, 
Japan) with dimensions $4.6 \mathrm{~mm} \times 150 \mathrm{~mm}$. A $50 \mu \mathrm{L}$ aliquot of the sample was injected at a flow rate of $0.6 \mathrm{~mL} / \mathrm{min}$.

Original chromatographic conditions employed for the determination of caffeine in green coffee extracts resulted in co-eluting peaks (Figure 3.1).

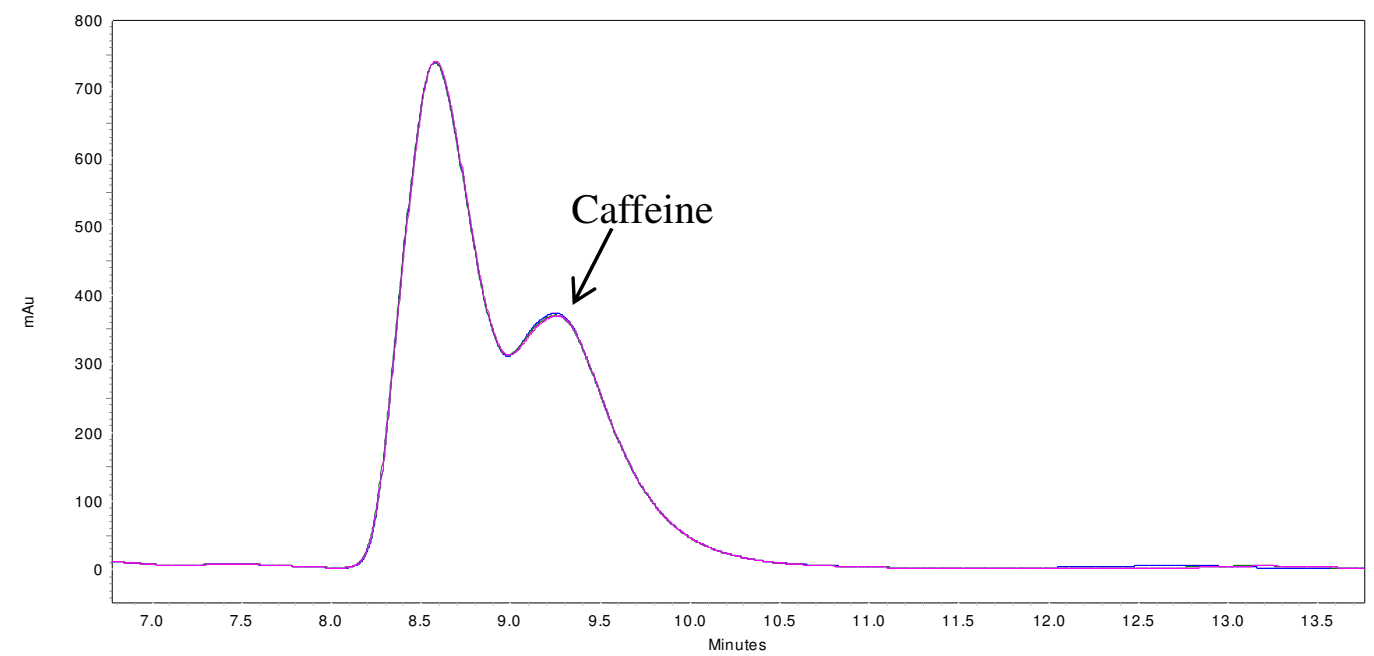

Figure 3.1. HPLC chromatogram of caffeine analysis $(280 \mathrm{~nm})$ from a green coffee extract diluted 1:50 with MPA.

Adequate peak separation and resolution is essential for proper peak integration and thus obtaining accurate results. Adjusting the gradient profile, in combination with other adjustments to the chromatographic conditions, attempts to effectively separate the peak co-eluting with caffeine. The original parameters for gradient elution, gradient "A", is depicted in Table 3.1. Table 3.2 shows gradient "B", the first attempt at resolving the caffeine peak by increasing to $12 \%$ solvent B at $\mathrm{t}=15$ minutes, $80 \%$ solvent $\mathrm{B}$ at $\mathrm{t}=35$ minutes and finally $100 \%$ solvent $\mathrm{B}$ at $\mathrm{t}=37$ minutes. This is in contrast to a slow linear increase to $100 \%$ solvent $\mathrm{B}$ at $\mathrm{t}=43$ minutes in the original gradient parameters. Gradient " $\mathrm{B}$ " also shortens the total run time to 62 minutes. Gradients "C" and "D" (Table 3.3 and Table 3.4) were slight modifications of gradient " $\mathrm{B}$ " at $\mathrm{t}=15$ minutes, holding all other Page 37 
conditions of the gradient profile the same. Gradient "E" (Table 3.5) employs the use of a step-gradient profile, increasing the percentage of solvent B quicker and held at $20 \%$ over a longer period of time. Table 3.6 shows the final gradient profile with a total run time of 60 minutes. Figure 3.2 shows all proposed gradient profiles in a graphical form. Mobile phase A was held at $100 \%$ for 3 minutes, and then changed by a linear gradient to $100 \% \mathrm{~B}$ over 30 minutes. The mobile phase was held at $100 \% \mathrm{~B}$ for 7 minutes, and a linear return to $100 \%$ A was made over 5 minutes. The mobile phase was held at $100 \%$ A for 18 minutes for reequilibration of the column prior to injecting a new sample. The composition of MPA and MPB remained unchanged from the original chromatographic conditions. Determination of the optimal gradient elution profile will be discussed in the results \& discussion section of this thesis.

Table 3.1. Time program of gradient "A"

\begin{tabular}{cc}
\hline Time (min) & \% Solvent B \\
\hline 0.01 & 0.0 \\
3.00 & 0.0 \\
43.00 & 100.0 \\
50.00 & 100.0 \\
60.00 & 0.0 \\
75.00 & 0.0 \\
76.00 & End \\
\hline
\end{tabular}

Table 3.2. Time program of gradient "B"

\begin{tabular}{cc}
\hline Time (min) & \% Solvent B \\
\hline 0.01 & 0.0 \\
3.00 & 0.0 \\
15.00 & 12.0 \\
35.00 & 80.0 \\
37.00 & 100.0 \\
42.00 & 100.0 \\
47.00 & 0.0 \\
62.00 & 0.0 \\
63.00 & End \\
\hline
\end{tabular}


Table 3.3. Time program of gradient " $\mathrm{C}$ "

\begin{tabular}{cc}
\hline Time (min) & \% Solvent B \\
\hline 0.01 & 0.0 \\
3.00 & 0.0 \\
15.00 & 10.0 \\
35.00 & 80.0 \\
37.00 & 100.0 \\
42.00 & 100.0 \\
47.00 & 0.0 \\
62.00 & 0.0 \\
63.00 & End \\
\hline
\end{tabular}

Table 3.4. Time program of gradient " $\mathrm{D}$ "

\begin{tabular}{cc}
\hline Time (min) & \% Solvent B \\
\hline 0.01 & 0.0 \\
3.00 & 0.0 \\
15.00 & 20.0 \\
35.00 & 80.0 \\
37.00 & 100.0 \\
42.00 & 100.0 \\
47.00 & 0.0 \\
62.00 & 0.0 \\
63.00 & End \\
\hline
\end{tabular}

Table 3.5. Time program of gradient "E"

\begin{tabular}{cc}
\hline Time (min) & \% Solvent B \\
\hline 0.01 & 0.0 \\
3.00 & 0.0 \\
4.00 & 20.0 \\
15.00 & 20.0 \\
35.00 & 80.0 \\
37.00 & 100.0 \\
42.00 & 100.0 \\
47.00 & 0.0 \\
62.00 & 0.0 \\
63.00 & End \\
\hline
\end{tabular}


Table 3.6. Time program of final gradient profile

\begin{tabular}{cc}
\hline Time (min) & \% Solvent B \\
\hline 0.01 & 0 \\
3.00 & 0 \\
30.00 & 100 \\
37.00 & 100 \\
42.00 & 0 \\
59.00 & 0 \\
60.00 & End \\
\hline
\end{tabular}

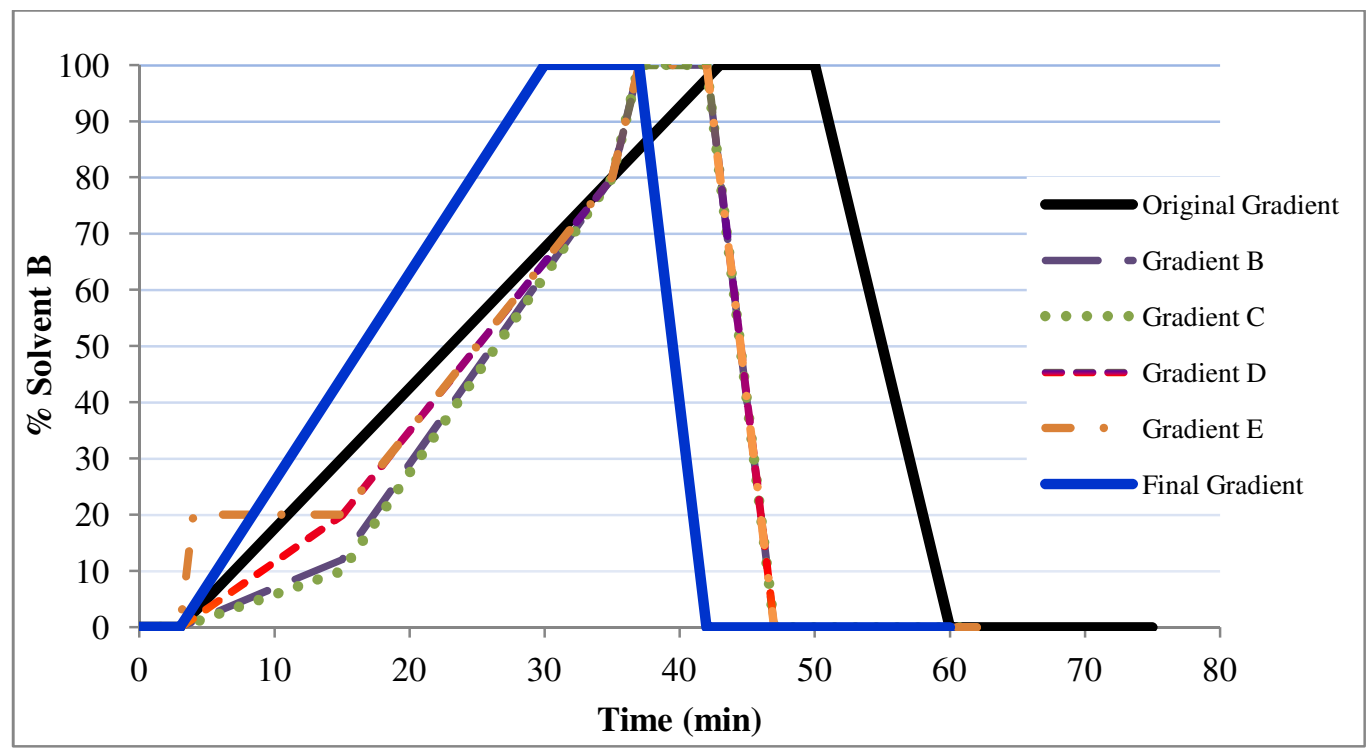

Figure 3.2. Gradient profiles for separation of caffeine by HPLC.

\subsubsection{Analytical column}

The original analytical column used for the analysis of caffeine and CGA in green coffee extracts was a Shimadzu $\mathrm{C}_{18}$ reversed phase (RP) column (Shimadzu, Japan) with dimensions $4.6 \mathrm{~mm}$ x $150 \mathrm{~mm}$. Subsequent analyses using this column afforded poor peak separation, poor baseline resolution at 280 $\mathrm{nm}$ and consequently, improper caffeine peak integration. To mitigate these issues resulting in inaccurate quantification of caffeine, an Alltech Platinum $\mathrm{RP} \mathrm{C}_{18}$ Extended Polar Selectivity (EPS) (Grace Davison Discovery Sciences) column with dimensions $4.6 \mathrm{~mm}$ x $250 \mathrm{~mm}$ and particle size of $5 \mu \mathrm{m}$ (Figure 3.3) was 
selected due to its increased polarity and length which is known to improve peak separation. Aliquots of $50 \mu \mathrm{L}$ samples of green coffee extract diluted 1:400 with MPA were injected using the original analytical column and then with the new analytical column, holding all other chromatographic conditions constant. A comparison of the ability of the analytical columns to effectively separate, resolve, and result in proper peak integration was done visually by overlaying the chromatograms through the use of EZ-Start 7.3 Chromatography Software (Shimadzu, Japan). Justification of using the Alltech analytical column in place of the Shimadzu analytical column will be provided in the results \& discussion section of this thesis.

Figure 3.3. HPLC analytical columns. Top $=$ Shimadzu $R P C_{18}$ column, dimensions $4.6 \mathrm{~mm} \times 150 \mathrm{~mm}$; bottom = Alltech Platinum RP $C_{18}$ EPS column, dimensions $4.6 \mathrm{~mm} \times 250 \mathrm{~mm}$.

\subsubsection{Column temperature}

The column oven temperature in the original chromatographic conditions was set at $40{ }^{\circ} \mathrm{C}$. As part of further optimizing peak separation, column oven temperature was investigated in the range of $35^{\circ} \mathrm{C}, 40^{\circ} \mathrm{C}, 45^{\circ} \mathrm{C}$ and $50{ }^{\circ} \mathrm{C}$. A single sample of green coffee extract diluted 1:800 with MPA was injected at the 
above temperatures using the new analytical column, holding all other chromatographic conditions constant. The samples were analyzed at both $280 \mathrm{~nm}$ and $330 \mathrm{~nm}$ for separation of caffeine and CGA across all temperatures. Peak separation was evaluated visually by overlaying the chromatograms using EZStart 7.3 Chromatography Software (Shimadzu, Japan). Determination of the optimal column oven temperature will be discussed in the results $\&$ discussion section of this thesis.

\subsubsection{Development of a method for the simultaneous determination of caffeine and CGA in green coffee extracts by RP-HPLC}

The simultaneous determination of caffeine and 5-CGA was performed using reversed phase High Pressure Liquid Chromatography (RP-HPLC). The HPLC unit (Shimadzu Prominence, CBM-20A), depicted in Figure 3.4 was equipped with an autosampler (SIL-20A), column oven (CTO-20A), and a photodiode Array (PDA) detector (SPD-M20A). The column employed was a $\mathrm{C}_{18}$ EPS silica column with dimensions of $4.6 \mathrm{~mm} \times 250 \mathrm{~mm}$, and particle size of $5 \mu \mathrm{m}$ (Grace Davison Discovery Sciences). A $\mathrm{C}_{18}$-EPS cartridge system guard column was also used containing the same packing material as the analytical column, with dimensions $7.5 \mathrm{~mm}$ x $3 \mathrm{~mm}$ (Grace Davison Discovery Sciences). Two mobile phases were mixed by a serial dual-piston pump (LC-20AT). The composition of MPA was 90:10:0.1 (v/v/v) MilliQ Nanopure water/acetonitrile/formic acid while the composition of MPB was 75:25:0.1 (v/v/v) MilliQ Nanopure water/acetonitrile/formic acid. The column was equilibrated at a column oven temperature of $40{ }^{\circ} \mathrm{C}$ in MPA for 15 minutes and at a flow rate of 0.60 
$\mathrm{mL} / \mathrm{minute} .50 \mu \mathrm{L}$ of the sample was injected and the mobile phase was held at $100 \%$ A for 3 minutes, and then changed by a linear gradient to $100 \%$ B over 30 minutes. The mobile phase was held at $100 \% \mathrm{~B}$ for 7 minutes, and a linear return to $100 \%$ A was made over 5 minutes. The mobile phase was held at $100 \% \mathrm{~A}$ for 18 minutes for re-equilibration of the column prior to injecting a new sample. The total analysis run time was 60 minutes. The column was stored and washed in $100 \%$ acetonitrile. Caffeine was analyzed at a wavelength of $280 \mathrm{~nm}$ while 5CGA was analyzed at a wavelength of $330 \mathrm{~nm}$. Peak integration was performed using EZ-Start 7.3 Chromatography Software (Shimadzu, Japan). The developed analytical method was subsequently subjected to validation studies, discussed in the next section. 


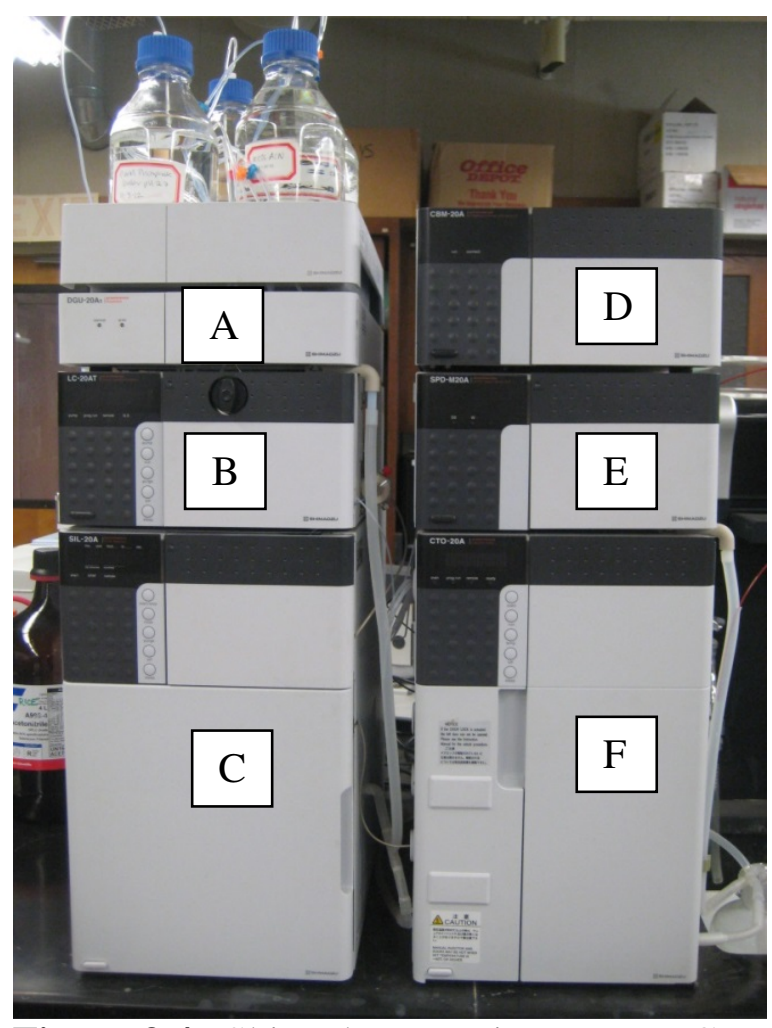

Figure 3.4. Shimadzu Prominence HPLC unit. A) degassing unit ( $\left.D G U-20 A_{3}\right)$; B) pumps (LC-20AT); C) autosampler (SIL-20A); D) communication bus module (CBM-20A); E) photo-diode array detector (SPD-M20A); F) column oven (CTO20A).

\subsection{HPLC Method validation studies}

\subsubsection{Linearity}

Calibration curves of the analytes, caffeine and 5-CGA, were constructed using commercial standards purchased from Sigma Aldrich (St. Louis, MO). A $1 \mathrm{mg} / \mathrm{mL}$ stock standard solution of each analyte was prepared in deionized (DI) water. The stock standard was then syringe filtered through a nylon filter $(13 \mathrm{~mm}$, $0.45 \mu \mathrm{m}$, Fisher Scientific). Serial dilutions of each standard were prepared to obtain standard concentrations of $0.125,0.0625,0.0313,0.0156,0.00781 \mathrm{mg} / \mathrm{mL}$. The standards were analyzed by RP-HPLC according to the newly developed analytical method mentioned previously. The peak areas were integrated using 
EZ-Start 7.3 Chromatography Software (Shimadzu, Japan). A calibration curve was constructed by plotting the peak areas of each dilution of the standard against its known concentration in Excel 2010 (Microsoft).

\subsubsection{Precision}

Commercial standards of caffeine and 5-CGA were used to prepare a $1 \mathrm{mg} / \mathrm{mL}$ stock standard solution. The stock standard was then syringe filtered through a nylon filter ( $13 \mathrm{~mm}, 0.45 \mu \mathrm{m}$, Fisher Scientific). Serial dilutions in DI water of each standard were prepared to obtain standard concentrations of 0.125 , 0.0625 and $0.0313 \mathrm{mg} / \mathrm{mL}$. Each concentration of the caffeine and 5-CGA standards were injected five times, from five separate amber HPLC vials, on a single day to result in 15 total determinations for each analyte. The standards were analyzed by RP-HPLC according to the newly developed analytical method mentioned previously. The peaks of each standard were integrated using EZ-Start 7.3 Chromatography Software (Shimadzu, Japan) and the relative standard deviation (\% RSD) of the peak areas were analyzed using Excel 2010 (Microsoft).

\subsubsection{Selectivity}

Preparation of a control sample containing all but the added bioactive compounds using the same sample procedure is necessary to establish selectivity of a method. A 3.4\% (w/v) sodium caseinate control sample was prepared by dissolving $6.8 \mathrm{~g}$ of sodium caseinate (lot\# ZC0027, 65\% protein, Spectrum Chemicals) in $50 \mathrm{~mL}$ of DI water while vigorously stirring with a stir bar. The solution was allowed to stir for approximately $1 \mathrm{~h}$, or until all the sodium caseinate had dissolved and resulted in a homogenous solution. An additional 50 
$\mathrm{mL}$ of DI water was then added to result in a $3.4 \%(\mathrm{w} / \mathrm{v})$ solution. Procedures for preparing sodium caseinate samples with caffeine and 5-CGA, outlined later, followed the above procedures, except the additional $50 \mathrm{~mL}$ of DI water was replaced with a $50 \mathrm{~mL}$ solution of caffeine or 5-CGA. Isoelectric precipitation of the sodium caseinate control sample was achieved by adjusting the $\mathrm{pH}$ of the sample to $\mathrm{pH} 4.6$ using $0.1 \mathrm{~N} \mathrm{HCl}$ (Fisher Scientific) at $30^{\circ} \mathrm{C}$. The sample was then centrifuged using an Eppendorf 5810 centrifuge (Hamburg, Germany) at 3,000 rpm, $20^{\circ} \mathrm{C}$ for $15 \mathrm{~min}$. The sample supernatant was then syringe filtered through a nylon filter (13 mm, $0.45 \mu \mathrm{m}$, Fisher Scientific) and analyzed by RPHPLC according to the newly developed analytical method mentioned previously.

\subsection{Binding interactions of caffeine and 5-CGA with sodium caseinate}

\subsubsection{Establishing a procedure for binding caffeine to sodium caseinate}

Given the limited research on the binding of caffeine and CGA to sodium caseinate, it was necessary to establish a protocol in order to carry out any further experiments. A procedure was established to bind caffeine and 5-CGA to sodium caseinate according to the methodology described by Zimet et al., (2011) with a few modifications. The molar ratio of sodium caseinate to caffeine was 1:1 and the molar ratio of sodium caseinate to 5-CGA was also 1:1.

\subsubsection{Preparation of caffeine stock standard}

A $0.264 \mathrm{mg} / \mathrm{mL}\left(1.36 \times 10^{-3} \mathrm{M}\right)$ caffeine standard (lot\#BCBF3955V, HPLC grade, Sigma Aldrich) was prepared in a volumetric flask dissolved in and diluted to volume with DI water. The same lot of caffeine standard was used throughout all experiments to reduce variations in the response. 


\subsubsection{Preparation of sodium caseinate sample}

A $6.8 \%(w / v)$ solution of sodium caseinate (lot\# ZC0027, 65\% protein, Spectrum Chemicals) was prepared by slowly adding approximately $34 \mathrm{~g}$ of sodium caseinate to a beaker containing $500 \mathrm{~mL}$ of DI water while vigorously stirring with a stir bar to create a vortex. This procedure was found to be more effective at dissolving the caseinate powder without adhering to the sides of the beaker. The solution was allowed to stir for approximately $1 \mathrm{~h}$, or until completely dissolved. Then $50 \mathrm{~mL}$ aliquots of the sodium caseinate solution was transferred into separate beakers and the combination of the treatment factors $\mathrm{pH}$ $(4.4,6.7)$ and temperature $\left(25^{\circ} \mathrm{C}, 72^{\circ} \mathrm{C}\right)$ were applied. Samples treated at $25^{\circ} \mathrm{C}$ were left at room temperature whereas samples treated at $72{ }^{\circ} \mathrm{C}$ were heated on a hot plate. The $\mathrm{pH}$ of the samples was then adjusted to $\mathrm{pH} 4.4$ or 6.7 using $0.1 \mathrm{~N}$ $\mathrm{HCl}$ (Fisher Scientific) or 0.1 N NaOH (Fisher Scientific). All subsequent experiments were performed using the same batch of sodium caseinate to reduce variations in the response. These procedures were also followed for experiments using 5-CGA.

\subsubsection{Preparation of 1:1 M ratio sodium caseinate to caffeine} samples

Caffeine stock standard solution $(50 \mathrm{~mL})$ was slowly added dropwise to a $50 \mathrm{~mL}$ aliquot of the homogenous $6.8 \%(\mathrm{w} / \mathrm{v})$ sodium caseinate solution applied with the treatments mentioned above while stirring to prepare a $3.4 \%(\mathrm{w} / \mathrm{v})$ caseinate sample with caffeine. The resulting sample had a final molar ratio of 1:1 sodium caseinate:caffeine. A control sample of $3.4 \%(\mathrm{w} / \mathrm{v})$ sodium caseinate was 
prepared by adding $50 \mathrm{~mL}$ of DI water to a $50 \mathrm{~mL}$ aliquot of the $6.8 \%(\mathrm{w} / \mathrm{v}$ )

sodium caseinate solution in a separate beaker while stirring at room temperature. A caffeine control sample $(0.132 \mathrm{mg} / \mathrm{mL}$ concentration $)$ was also prepared by adding $50 \mathrm{~mL}$ of DI water to $50 \mathrm{~mL}$ of caffeine stock standard at room temperature. The samples were then allowed to stir for $1 \mathrm{~h}$, with the temperature of the heated samples maintained between $65-75^{\circ} \mathrm{C}$. At the end of the hour, the $\mathrm{pH}$ of the samples were measured and recorded. All samples were prepared in duplicate.

\subsubsection{Analytical fractionation}

Isoelectric precipitation of the caseins in the samples was achieved by heating the samples prepared at $25{ }^{\circ} \mathrm{C}$ to $30-35^{\circ} \mathrm{C}$ on a hot plate while stirring and slowly adjusting the $\mathrm{pH}$ to 4.6 using $0.1 \mathrm{~N} \mathrm{HCl}$ or $0.1 \mathrm{~N} \mathrm{NaOH}$. Samples prepared at $72{ }^{\circ} \mathrm{C}$ were cooled to $30-35^{\circ} \mathrm{C}$, and then the $\mathrm{pH}$ of the samples were adjusted to $\mathrm{pH} 4.6$ using $0.1 \mathrm{~N} \mathrm{HCl}$ or $0.1 \mathrm{~N} \mathrm{NaOH}$. The entire sample was then quantitatively transferred into suitable centrifuge bottles and centrifuged at 3,000 $\mathrm{rpm}, 20^{\circ} \mathrm{C}$ for $15 \mathrm{~min}$ to obtain a casein pellet. The volume of the resulting supernatant was measured. These procedures were also followed for experiments using 5-CGA. All samples were wrapped in foil to protect from light degradation.

\subsubsection{Establishing a procedure for binding 5-CGA to sodium caseinate}

\subsubsection{Preparation of 5-CGA stock standard}

A $0.482 \mathrm{mg} / \mathrm{mL} 5-C G A$ (lot\#SLBB6914V, >95\% titration, Sigma Aldrich) standard was prepared in a volumetric flask dissolved in and diluted to volume with DI 
water. The same lot of 5-CGA was used throughout all experiments to reduce variations in the response.

\subsubsection{Preparation of 1:1 M ratio sodium caseinate to 5-CGA samples}

5-CGA stock standard solution $(50 \mathrm{~mL})$ was added dropwise to a $50 \mathrm{~mL}$ aliquot of the homogenous $6.8 \%(\mathrm{w} / \mathrm{v})$ sodium caseinate solution applied with the treatments mentioned previously while stirring to prepare a $3.4 \%(\mathrm{w} / \mathrm{v})$ caseinate sample with 5-CGA. The resulting sample had a final molar ratio of 1:1 sodium caseinate:5-CGA. A control sample of 3.4\% (w/v) sodium caseinate was prepared by adding $50 \mathrm{~mL}$ of DI water to $50 \mathrm{~mL}$ of the $6.8 \%(\mathrm{w} / \mathrm{v})$ sodium caseinate solution in a separate beaker while stirring at room temperature. A 5-CGA control sample $(0.241 \mathrm{mg} / \mathrm{mL}$ concentration $)$ was also prepared by adding $50 \mathrm{~mL}$ of DI water to $50 \mathrm{~mL}$ of stock 5-CGA standard at room temperature. The sample was then allowed to stir for $1 \mathrm{~h}$ with the temperature of heated samples maintained between $65-75^{\circ} \mathrm{C}$. At the end of the hour the $\mathrm{pH}$ of the samples were measured and recorded. All samples were prepared in duplicate.

\subsubsection{Analytical fractionation}

Procedures for isoelectric precipitation of caseins in samples containing 5CGA followed the same methodology as described previously for isoelectric precipitation of caseins in samples containing caffeine, outlined above.

\subsection{Experimental design}

A Generalized Randomized Complete Block Design (GRCBD), blocked by day, was employed in a $2^{2}$ full factorial design with two replicates per block. 
The treatment factors were $\mathrm{pH}$ and temperature, each at two levels (Table 3.7). The experimental unit was a sample of a 1:1 $\mathrm{M}$ ratio of sodium caseinate to caffeine or 5-CGA. Treatments were randomly assigned to the experimental unit using Excel 2010 (Microsoft) (Table 3.8 \& Table 3.9). All treatments were applied to aliquots of the $6.8 \%(\mathrm{w} / \mathrm{v})$ sodium caseinate solution prior to adding any caffeine or 5-CGA. The response was the concentration of bioactive compound detected in the supernatant after centrifugation using HPLC.

Table 3.7. Levels of the treatment factors

\begin{tabular}{ll}
\hline Treatment & Levels \\
\hline $\mathrm{pH}$ & $4.4,6.7$ \\
Temperature & $25^{\circ} \mathrm{C}, 72^{\circ} \mathrm{C}$ \\
\hline
\end{tabular}


Table 3.8. Randomization of treatments to experimental unit in caffeine samples

\begin{tabular}{cccc}
\hline Run Order & pH & Temperature $\left({ }^{\circ} \mathbf{C}\right)$ & Block (Day) \\
\hline 1 & 6.7 & 25 & 1 \\
2 & 6.7 & 25 & 1 \\
3 & 6.7 & 72 & 1 \\
4 & 4.4 & 25 & 1 \\
5 & 4.4 & 72 & 1 \\
6 & 4.4 & 72 & 1 \\
7 & 6.7 & 72 & 1 \\
8 & 4.4 & 25 & 1 \\
9 & 6.7 & 25 & 2 \\
10 & 4.4 & 72 & 2 \\
11 & 6.7 & 72 & 2 \\
12 & 4.4 & 25 & 2 \\
13 & 6.7 & 72 & 2 \\
14 & 6.7 & 25 & 2 \\
15 & 4.4 & 25 & 2 \\
16 & 4.4 & 72 & 2 \\
\hline
\end{tabular}

Table 3.9. Randomization of treatments to experimental unit in 5-CGA samples

\begin{tabular}{cccc}
\hline Run Order & pH & Temperature $\left({ }^{\circ} \mathbf{C}\right)$ & Block (Day) \\
\hline 1 & 6.7 & 25 & 1 \\
2 & 6.7 & 72 & 1 \\
3 & 6.7 & 25 & 1 \\
4 & 4.4 & 72 & 1 \\
5 & 4.4 & 25 & 1 \\
6 & 4.4 & 25 & 1 \\
7 & 6.7 & 72 & 1 \\
8 & 4.4 & 72 & 1 \\
9 & 4.4 & 25 & 2 \\
10 & 6.7 & 25 & 2 \\
11 & 4.4 & 72 & 2 \\
12 & 6.7 & 72 & 2 \\
13 & 4.4 & 25 & 2 \\
14 & 6.7 & 72 & 2 \\
15 & 6.7 & 25 & 2 \\
16 & 4.4 & 72 & 2 \\
\hline
\end{tabular}




\subsection{Application of established protocol and optimal treatment conditions on green coffee extract}

A permeate extract of green coffee was obtained from the liquid extraction of Arabica green coffee beans from Brazil using water. The extract was passed through a filtration system equipped with a membrane filter with a 5,000 molecular weight cut-off. Lastly, the extract was passed through an ultrafiltration system (GEA RT-12) using the maximum cross flow ratio setting to achieve a flow rate of $\sim 20 \mathrm{~L} / \mathrm{min}$. A clear, yellow permeate was obtained and used as the green coffee extract for further experiments (Figure 3.5). A 6.8\% (w/v) sodium caseinate solution $(50 \mathrm{~mL})$ was prepared as previously described and heated to 72 ${ }^{\circ} \mathrm{C}$ on a hotplate while stirring. The $\mathrm{pH}$ of the solution was then adjusted to $\mathrm{pH}$ 6.7 using $0.1 \mathrm{~N} \mathrm{HCl}$. Then $50 \mathrm{~mL}$ of the green coffee extract was then slowly added to the sodium caseinate solution dropwise while stirring. The sample was allowed to stir for $1 \mathrm{~h}$ with the temperature maintained between $65-75{ }^{\circ} \mathrm{C}$. The beaker used to contain the sample was wrapped in foil to protect from light degradation. The sample was cooled to $30-35^{\circ} \mathrm{C}$ before adding $0.1 \mathrm{~N} \mathrm{HCl}$ to the isoelectric point ( $\mathrm{pH} 4.6$ ) while stirring. The entire contents of the sample were quantitatively transferred into a suitable centrifuge bottle and centrifuged at 3,000 $\mathrm{rpm}, 20^{\circ} \mathrm{C}$ for $15 \mathrm{~min}$. The volume of the supernatant was measured using a graduated cylinder. The sample supernatant was retained for HPLC and BCA analysis. Each sample was syringe filtered through a nylon filter $(13 \mathrm{~mm}, 0.45$ $\mu \mathrm{m}$, Fisher Scientific) and transferred into amber HPLC vials for analysis. The sample was prepared in triplicate. 


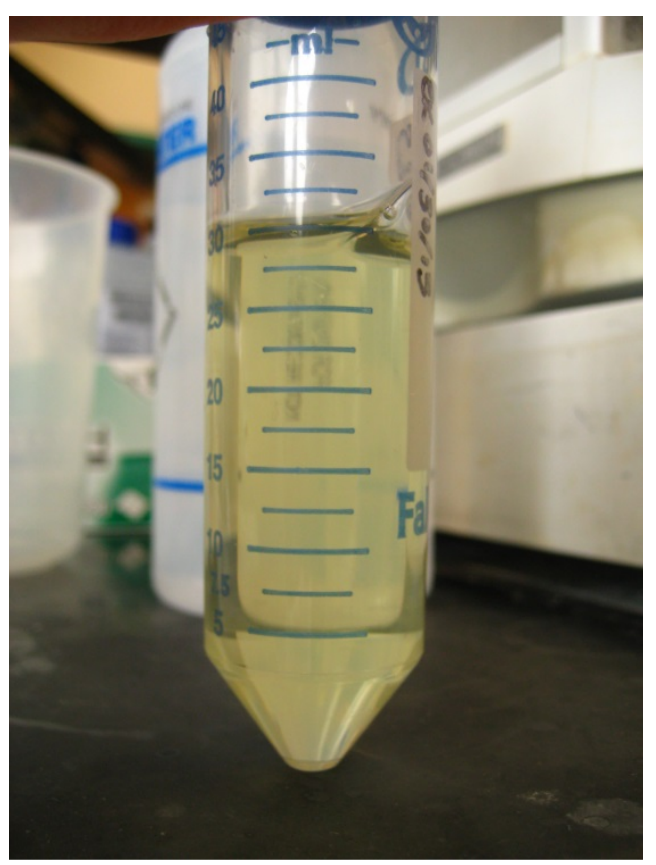

Figure 3.5. Permeate of green coffee extract obtained after ultrafiltration.

\subsection{Statistical analysis}

A Generalized Randomized Complete Block Design (GRCBD) blocked by day was employed in a $2^{2}$ full factorial design performed in duplicate. The treatment factors were $\mathrm{pH}(4.4,6.7)$ and temperature $\left(25^{\circ} \mathrm{C}, 72^{\circ} \mathrm{C}\right)$. The response was the concentration of caffeine or 5-CGA detected in the supernatant of samples using HPLC. All data analysis was carried out using the statistical software, JMP Pro 10 (SAS Institute Inc.) with significance at $\mathrm{p}<0.01$.

\subsection{HPLC analysis of caffeine and 5-CGA}

HPLC analysis of caffeine and 5-CGA was performed following the developed method outlined previously in this thesis. All samples were syringe filtered through a nylon filter $(13 \mathrm{~mm}, 0.45 \mu \mathrm{m}$, Fisher Scientific) and a $1.0 \mathrm{~mL}$ aliquot was transferred into amber HPLC vials for analysis. Samples were analyzed in the randomized order previously determined for sample preparation 
(see Tables 3.8 and 3.9). All samples were analyzed on the same day of preparation to minimize any degradation of the bioactive compound. Peak integration was performed using EZ-Start 7.3 Chromatography Software (Shimadzu, Japan). All data was collected and analyzed using Excel 2010 (Microsoft). The concentration of caffeine and 5-CGA in the samples was calculated through the use of standard calibration curves of each compound prepared at known concentrations.

\subsection{BCA protein assay}

Bicinchoninic acid (BCA) analysis was performed following instructions provided by the Pierce BCA ${ }^{\mathrm{TM}}$ Protein Assay Kit (No. 23227, Rockford, IL). A standard curve was prepared using Bovine Serum Albumin (BSA) (Product No. 23209, $2 \mathrm{mg} / \mathrm{mL}$ concentration) standard in $0.9 \%$ saline and $0.05 \%$ sodium azide in the concentration range of $2.0-0.025 \mathrm{mg} / \mathrm{mL}$ in DI water. DI water was also used as the blank. The working reagent (WR) was prepared by mixing Reagent A (Product No. 23228, containing sodium carbonate, sodium bicarbonate, bicinchoninic acid and sodium tartrate in $0.1 \mathrm{M}$ sodium hydroxide) with Reagent B (Product No. 1859078, containing 4\% cupric sulfate) in a 50:1 ratio, respectively.

Samples were randomly selected from each treatment combination of caffeine and 5-CGA with sodium caseinate samples for BCA analysis (Table 3.10), along with the sodium caseinate solution $(6.8 \%(\mathrm{w} / \mathrm{v}))$ and sodium caseinate control $(3.4 \%(\mathrm{w} / \mathrm{v}))$ containing neither caffeine nor 5-CGA. The sodium caseinate solution was diluted three times (1:25, 1:50 and 1:100) using DI 
water to ensure a measurable absorbance was obtained. All other samples were analyzed without any further dilution. A 1:20 sample:WR ratio was used for the preparation of BCA samples. $2.0 \mathrm{~mL}$ of $\mathrm{WR}$ was added into separate $15 \mathrm{~mL}$ Falcon tubes. An aliquot of $100 \mu \mathrm{L}$ of each standard and sample was then added separately to the tubes, capped and mixed well. The samples were incubated at $37^{\circ} \mathrm{C}$ for 30 minutes, cooled to room temperature, and measured using a SpectraMax Plus spectrophotometer (Sunnyvale, CA) at $562 \mathrm{~nm}$ within 10 minutes. All samples were analyzed in duplicate. The absorbance of the blank solution was subtracted from the absorbance of each standard and sample prior to analyzing the data in Excel 2010 (Microsoft).

Table 3.10. Randomly selected samples and their treatment combinations

\begin{tabular}{|c|c|c|}
\hline Sample & $\mathbf{p H}$ & Temperature $\left({ }^{\circ} \mathbf{C}\right)$ \\
\hline \multirow{3}{*}{ Sodium caseinate \& } & 6.7 & 25 \\
\cline { 2 - 3 } caffeine & 6.7 & 72 \\
\cline { 2 - 3 } & 4.4 & 25 \\
\cline { 2 - 3 } & 4.4 & 72 \\
\hline \multirow{3}{*}{ Sodium caseinate \& } & 6.7 & 25 \\
\cline { 2 - 3 } 5-CGA & 6.7 & 72 \\
\cline { 2 - 3 } & 4.4 & 25 \\
\cline { 2 - 3 } & 4.4 & 72 \\
\hline
\end{tabular}




\subsection{RESULTS AND DISCUSSION}

\subsection{Development of a method for the simultaneous determination of caffeine and CGA in green coffee extracts by RP-HPLC}

\subsubsection{Determination of HPLC chromatographic conditions}

The principal objective of these experiments was to develop an HPLC method for the quantification of caffeine and 5-CGA in green coffee extracts. Eventually, this method would be extended to sodium caseinate samples containing both these analytes. Caffeine and 5-CGA are both of relatively low molecular weights $(<2000)$ and are both soluble in the mobile phases employed indicating that RP-HPLC is a suitable choice for quantification. Three main parameters of the chromatographic conditions evaluated for optimization were: gradient profile, analytical column, and column temperature. Optimization within each of these conditions resulted in the final developed RP-HPLC method.

\subsubsection{Gradient profile}

Four variations of the original gradient profile were evaluated for the purpose of separating caffeine and its co-eluting peak, in addition to reducing total run time. While only changing the gradient profile did not result in successful separation of the caffeine peak, the total run time was decreased from 72 min to 60 min (Figure 4.1). Eluent strength decreases with an increase in polarity of the mobile phase. Thus the reduction in time to reach $100 \%$ solvent B in the final gradient profile provided better separation of the caffeine and coeluting peak when combined with optimization to the column and column temperature. In addition, a closer inspection of Figure 4.1 shows a slight increase 
in peak sharpness was observed in the final gradient profile when compared to the original (Figure 4.2). The other variations of the gradient profiles B-E resulted in only slight changes from the original gradient profile (chromatograms not shown).

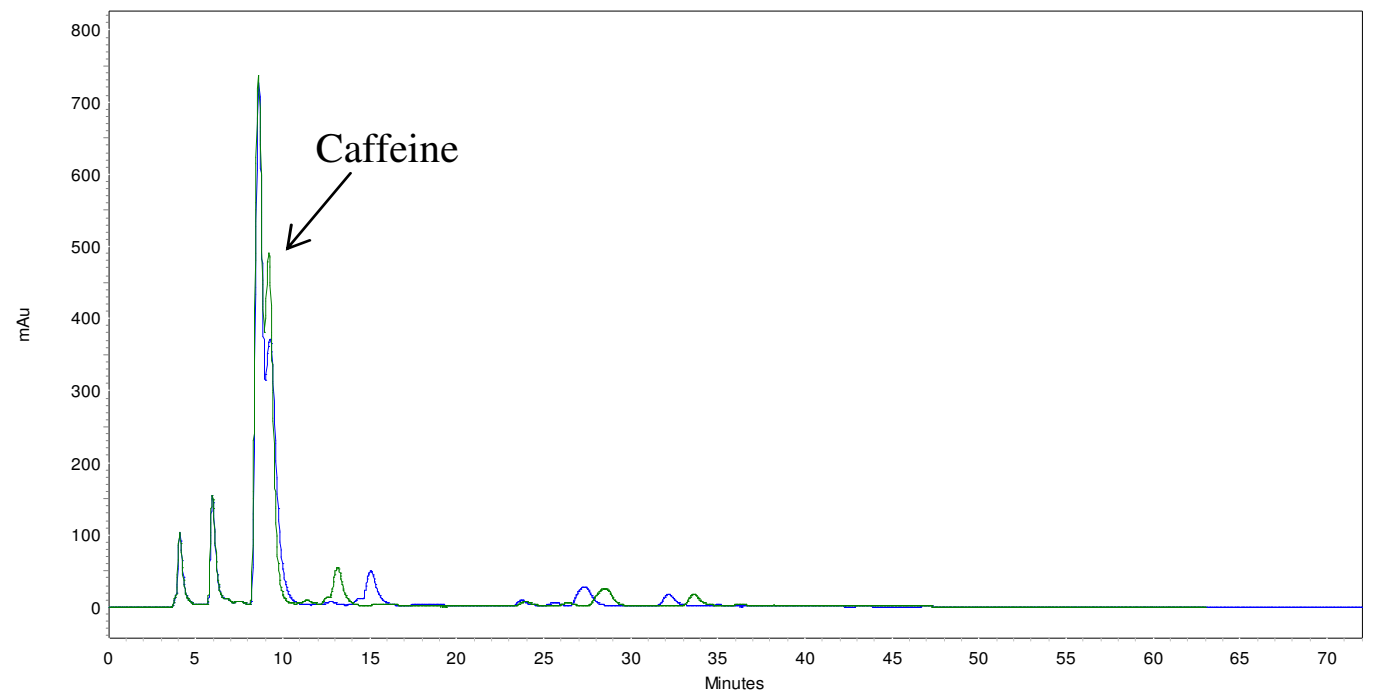

Figure 4.1. Overlay of green coffee extract. Sample diluted 1:50 with MPA analyzed at $280 \mathrm{~nm}$. The blue trace is the original gradient profile with total run time $72 \mathrm{~min}$, the green trace is the new gradient profile with total run time $60 \mathrm{~min}$.

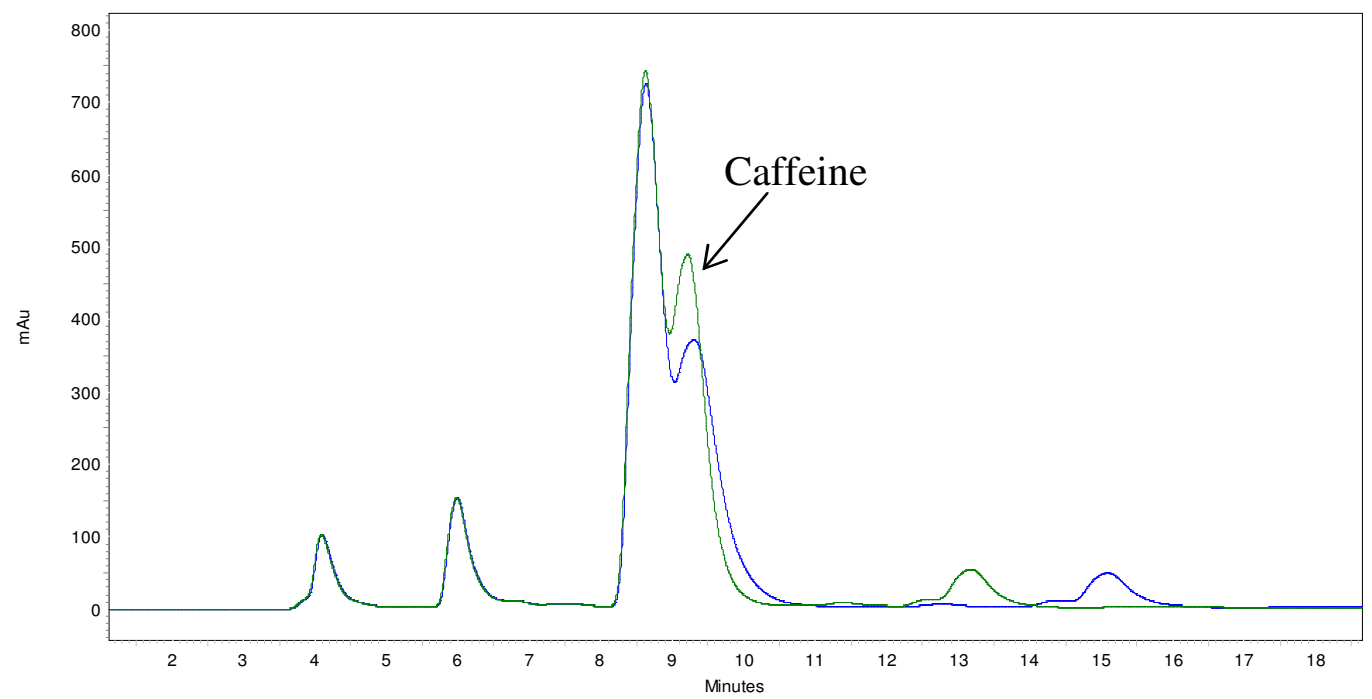

Figure 4.2. Slight peak sharpening observed in caffeine peak using new gradient profile (green trace) vs. old gradient profile (blue trace). 


\subsubsection{Analytical column}

The next parameter evaluated was the analytical column. The packing material in both columns was composed of a bonded silica surface covalently attached with octadecyl $\left(\mathrm{C}_{18}\right)$ chains, resulting in a nonpolar stationary phase. A nonpolar stationary phase used in RP-HPLC concurrent with gradient elution provides rapid elution of polar compounds carried via a polar mobile phase and slower elution of nonpolar compounds that have a greater affinity to interact with the nonpolar bonded silica surface. Therefore, increasing the length of the nonpolar stationary phase results in enhanced separation of polar and nonpolar (or slightly less polar) compounds by increasing interactions sites available to the nonpolar analyte. An increase of just $100 \mathrm{~mm}$ of the column length from the Shimadzu $\mathrm{C}_{18}$ RP column (length $150 \mathrm{~mm}$ ) to the Alltech Platinum $\mathrm{C}_{18}$ RP-EPS column (length $250 \mathrm{~mm}$ ), as well as the difference in packing material of the columns provided superior separation of the caffeine peak (Figure 4.3).

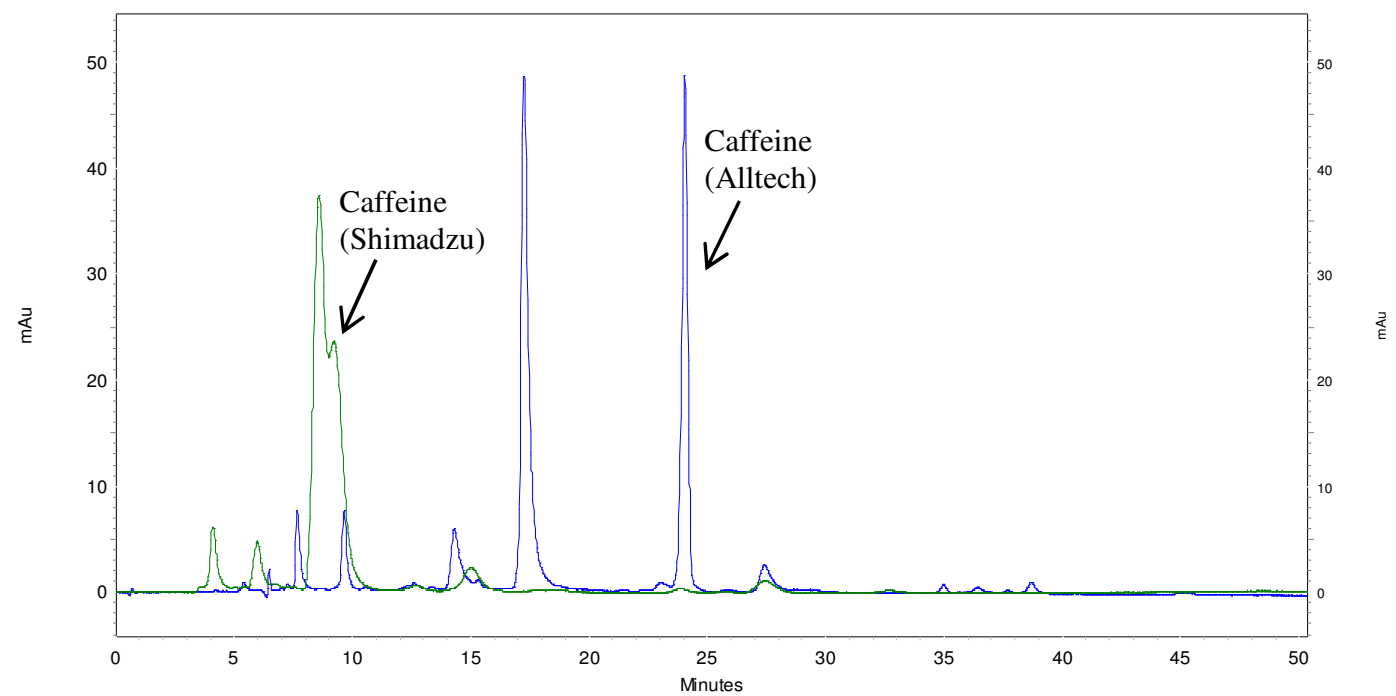

Figure 4.3. Overlay of green coffee extract (diluted 1:400 with MPA) using Shimadzu (green trace) and Alltech column (blue trace) for caffeine analysis. 


\subsubsection{Column temperature}

Control of column temperature in HPLC is essential for improved retention time reproducibility and precision of a quantitative analysis. Increases in column temperature provide a decrease in retention time and improves baseline resolution by accelerating diffusion of solutes. However, increases in column temperature generally also results in quicker degradation of the stationary phase thereby decreasing column lifetime. Therefore, it is imperative to determine the optimal column temperature for adequate elution and resolution of an analyte that will also result in greatest column lifetime. Four column temperatures: $35{ }^{\circ} \mathrm{C}$, $40{ }^{\circ} \mathrm{C}, 45^{\circ} \mathrm{C}$, and $50{ }^{\circ} \mathrm{C}$ were investigated in an attempt to optimize elution time of the caffeine and 5-CGA peaks concurrent with the optimized gradient profile and updated selection of the analytical column (Figure $4.4 \&$ Figure 4.5).

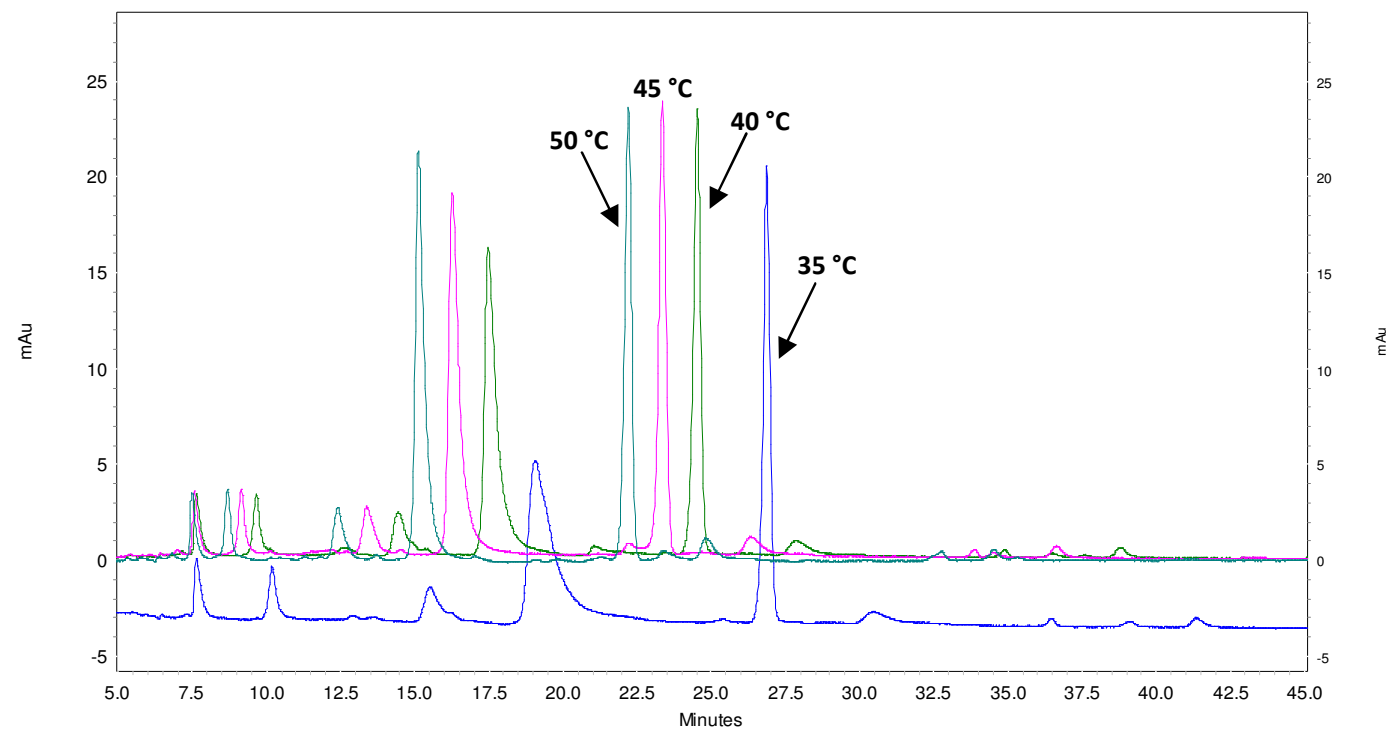

Figure 4.4. Overlay of caffeine peak at $280 \mathrm{~nm}$. From left to right: caffeine peak measured at $50^{\circ} \mathrm{C}, 45^{\circ} \mathrm{C}, 40^{\circ} \mathrm{C}$, and $35^{\circ} \mathrm{C}$. 


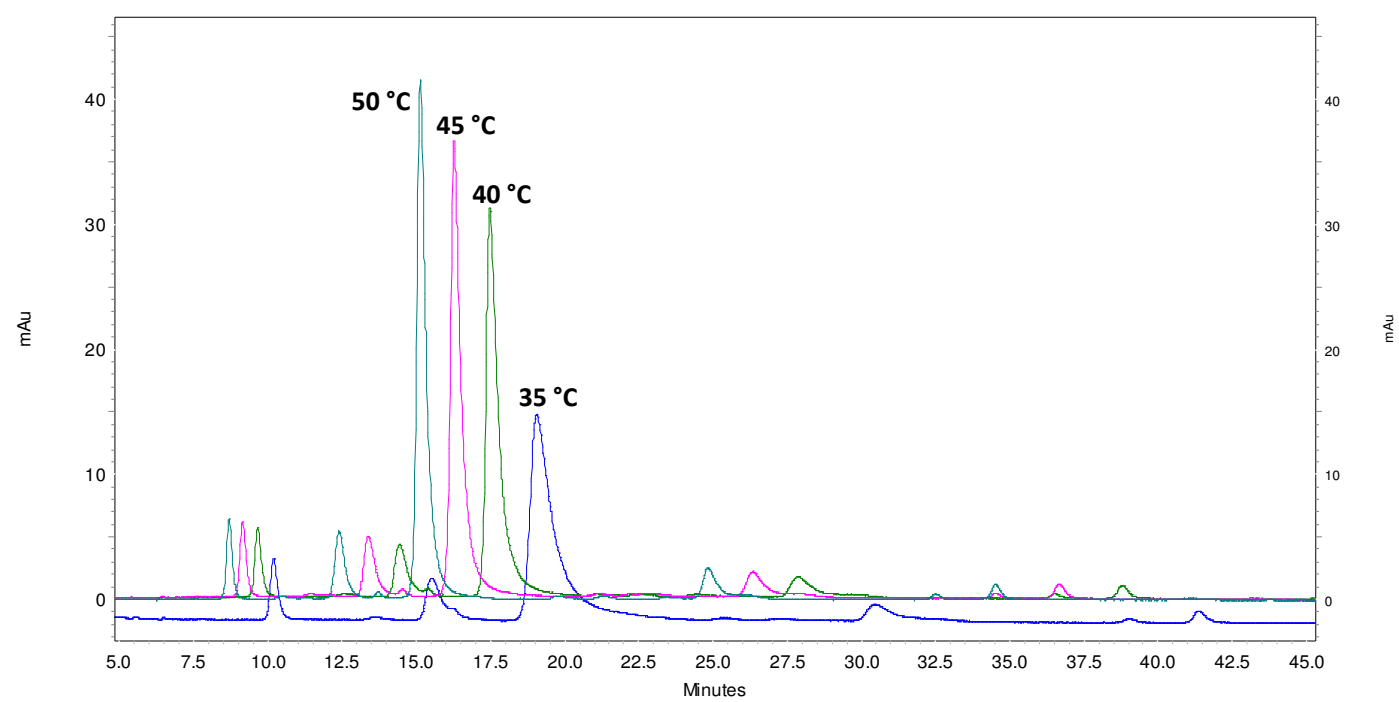

Figure 4.5. Overlay of 5-CGA peak at $330 \mathrm{~nm}$. From left to right: CGA pkea measured at $50^{\circ} \mathrm{C}, 45^{\circ} \mathrm{C}, 40^{\circ} \mathrm{C}$, and $35^{\circ} \mathrm{C}$.

Elution of the caffeine peak was observed to decrease from approximately $\mathrm{RT}=27 \mathrm{~min}$ at $35^{\circ} \mathrm{C}$ to approximately $\mathrm{RT}=22 \mathrm{~min}$ at $50{ }^{\circ} \mathrm{C}$, a total reduction of 5 minutes in retention time. A slight general increase in peak sharpness and resolution was also observed with an increase in column temperature. In addition, improved peak separation was observed in an unknown peak containing a shoulder at approximately $\mathrm{RT}=2.5 \mathrm{~min}$ at $50^{\circ} \mathrm{C}$ compared with the same sample injected at $40{ }^{\circ} \mathrm{C}$ (Figure 4.6). However, as mentioned previously, there must be a balance between retention time and column lifetime. Therefore, column temperature at $40{ }^{\circ} \mathrm{C}$ was determined the optimal temperature that provided adequate peak elution and resolution without subjecting the column to severe degradation at the higher temperatures investigated. 


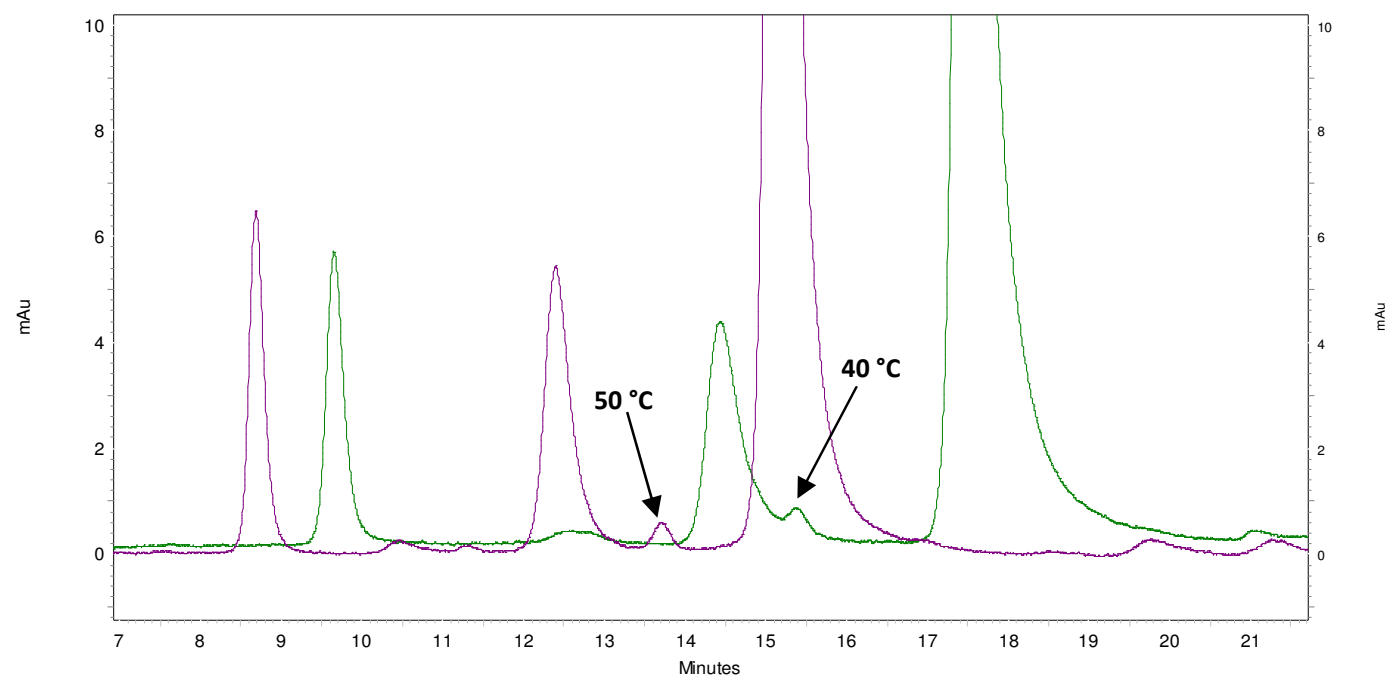

Figure 4.6. Separation of unknown peak with shoulder at $50^{\circ} \mathrm{C}$ (fully resolved) compared to $40^{\circ} \mathrm{C}$ (co-eluting) at $330 \mathrm{~nm}$.

\subsection{HPLC Method Validation}

\subsubsection{Linearity}

Linearity is the test of a method's ability to quantitatively analyze a sample and obtain results that are directly proportional to a known concentration of the analyte within that sample. Many factors such as age of the detector, adsorption of the analyte to the surface of the sample vial, degradation of the sample upon sitting, and phase separation of the sample can lead to under or over estimation of the amount of the analyte in a sample and can result in nonlinear results. Therefore, the importance of verifying the linearity of a method is a crucial step in method development.

The linearity study was carried out by preparing a stock caffeine and 5CGA standard solution of high concentration $(1 \mathrm{mg} / \mathrm{mL})$ and performing serial dilutions using a micropipette to a known concentration range resulting in five standards (Table 4.1). A graph of peak area vs. concentration was plotted and a 
least squares fit regression line was constructed (Figure 4.7 and Figure 4.8). A correlation coefficient $\mathrm{R}^{2} \geq 0.980$ is considered an acceptable result for linearity. A correlation coefficient $\mathrm{R}^{2}<0.980$ indicates nonlinearity of the method. The $\mathrm{R}^{2}$ value for caffeine and 5-CGA was 0.9992 and 0.9995, respectively. This demonstrates linearity of the developed RP-HPLC method.

Table 4.1. Concentration range and peak areas of caffeine and 5-CGA used in linearity study

\begin{tabular}{cccc}
\hline Dilution & $\begin{array}{c}\text { Concentration } \\
(\mathbf{m g} / \mathbf{m L})\end{array}$ & $\begin{array}{c}\text { Caffeine peak } \\
\text { area }\end{array}$ & $\begin{array}{c}\text { 5-CGA peak } \\
\text { area }\end{array}$ \\
\hline $1: 8$ & 0.125 & 21909584 & 27548226 \\
$1: 16$ & 0.0625 & 11563744 & 13116460 \\
$1: 32$ & 0.0313 & 5869057 & 6249741 \\
$1: 64$ & 0.0156 & 2946908 & 2993404 \\
$1: 128$ & 0.00781 & 1474020 & 1441236 \\
\hline
\end{tabular}

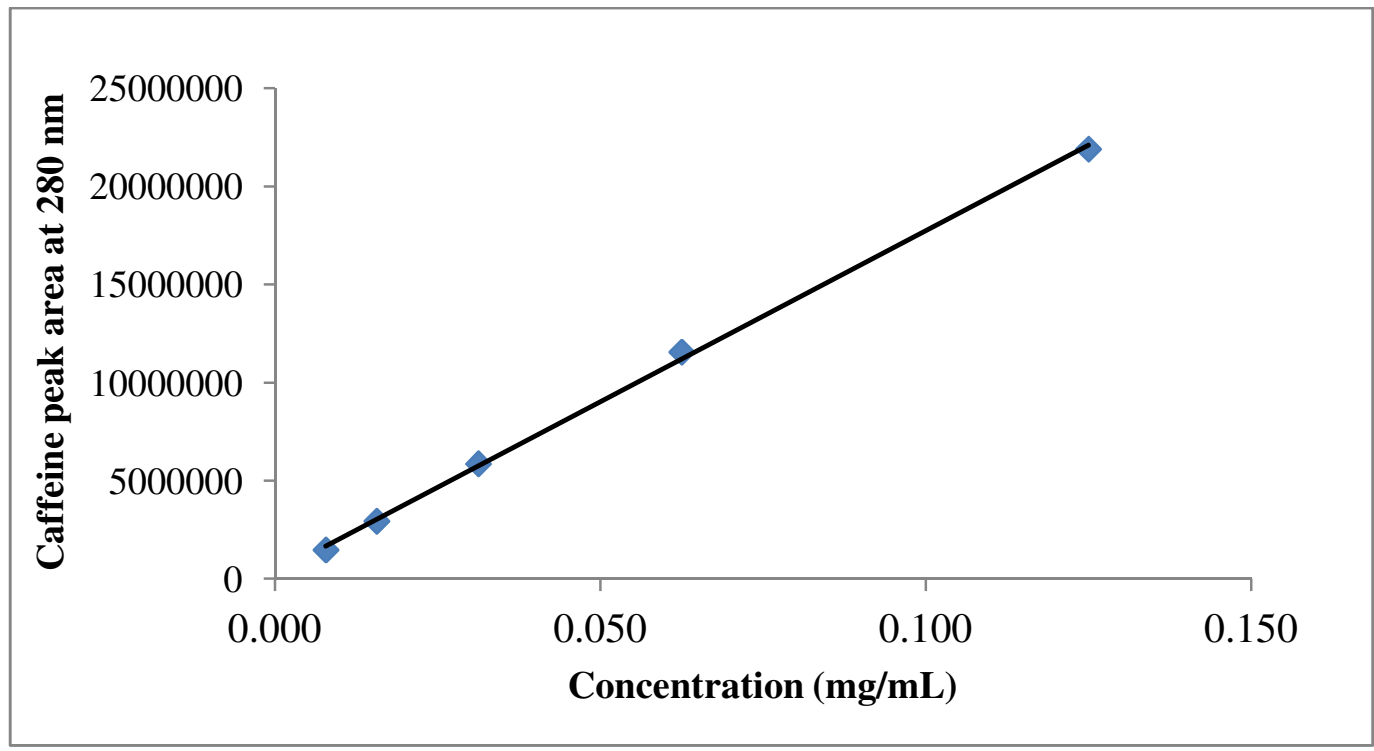

Figure 4.7. Graph of caffeine peak area (mAU) vs. concentration $(\mathrm{mg} / \mathrm{mL})$ in linearity study. $R^{2}=0.9992$. 


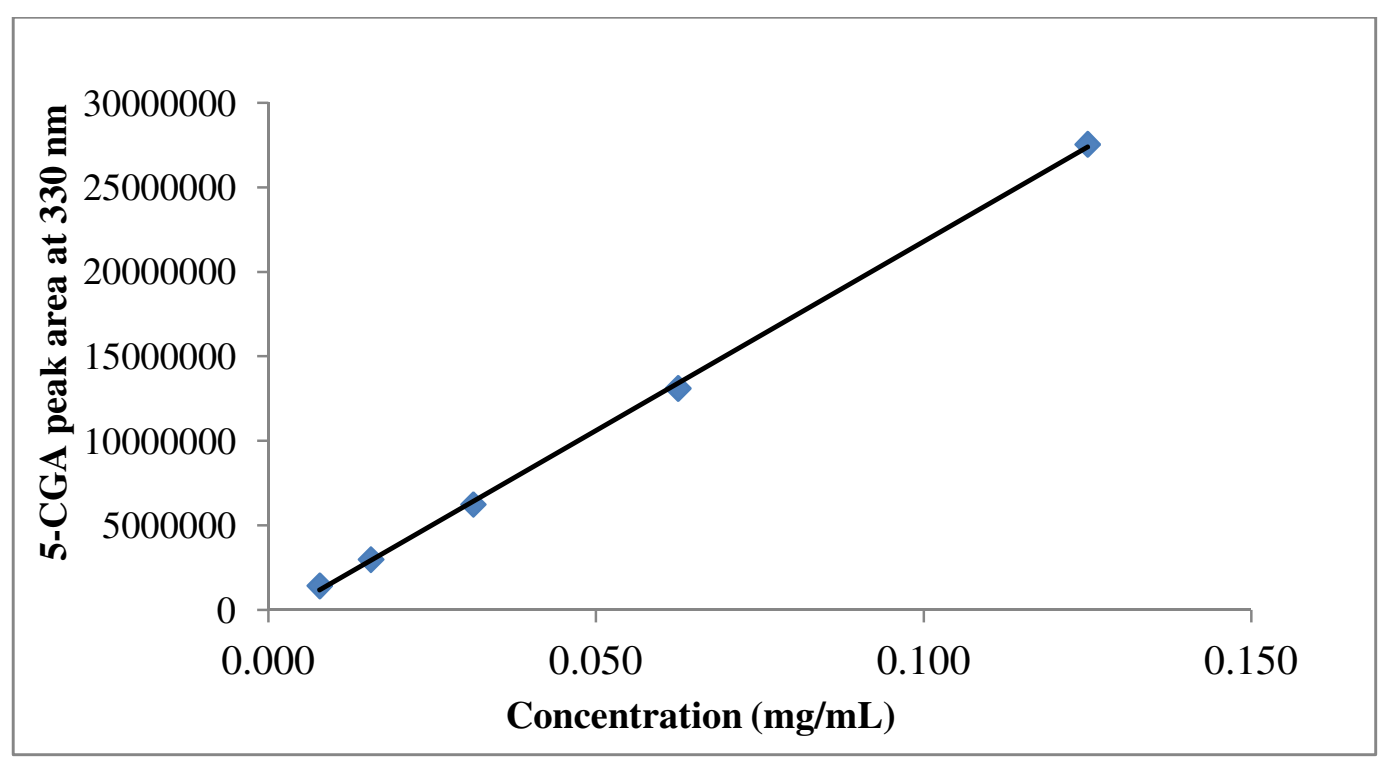

Figure 4.8. Graph of 5-CGA peak area (mAU) vs. concentration $(\mathrm{mg} / \mathrm{mL})$ in linearity study. $R^{2}=0.9995$.

\subsubsection{Precision}

Precision is a measure of the closeness of agreement (degree of scatter) between a series of measurements of the same homogeneous sample analyzed at set chromatographic conditions. Intra-assay precision, or within-day precision, was evaluated for the precision study. Five separate injections of three standard concentrations of caffeine and 5-CGA were each measured within the same day (Figure 4.9). The relative standard deviation (\% RSD) between the five injections of each standard concentration was calculated to determine the precision of the method. Acceptable precision ranges for HPLC methods are between $<2-10 \%$, with $<2 \%$ RSD being a method with great precision, $<5 \%$ RSD being a method with good precision, and $<10 \%$ RSD being a method with tolerable precision. An RSD value $>10 \%$ indicates a method with poor precision. The average RSD was $<1 \%$ and $<2 \%$ for caffeine and 5-CGA, respectively. These results indicate great precision of the developed method (Appendix A).

\section{Page 63}




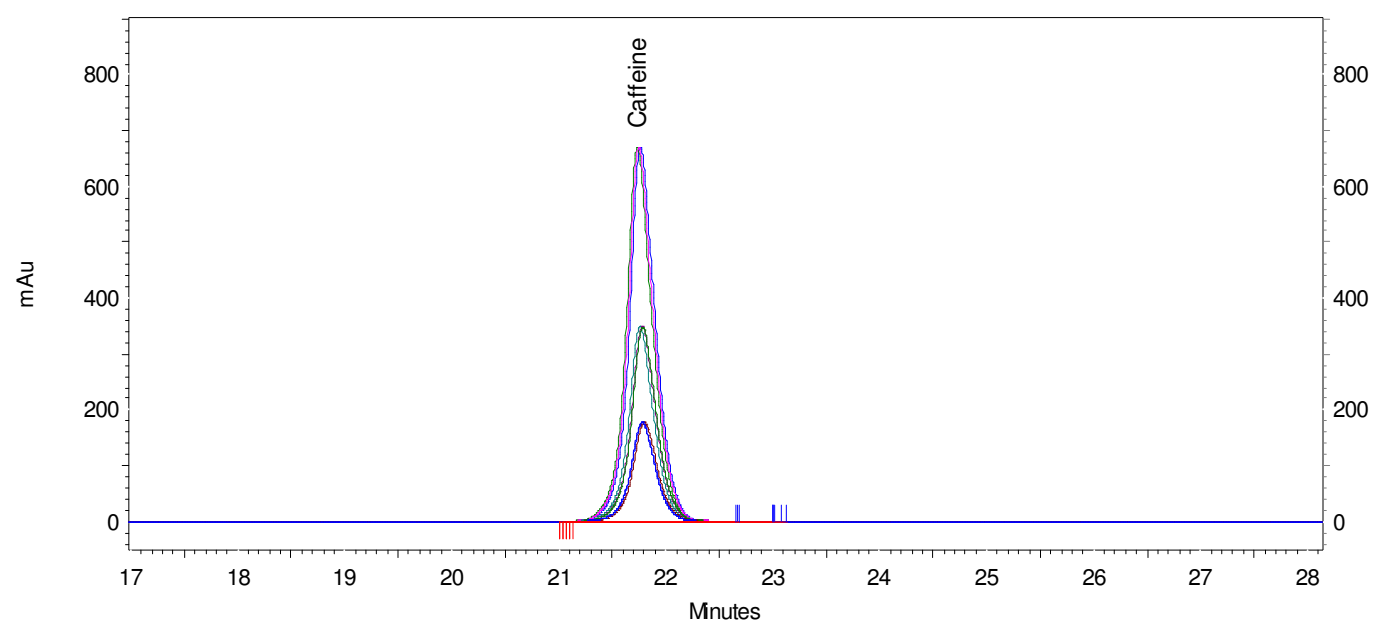

Figure 4.9. Overlay of caffeine standard at three concentrations. Five injections for each sample, $R S D<1 \%$ for all concentrations. Note chromatogram zoomed in to show time between 17-28 min only (total run time $60 \mathrm{~min}$ ).

\subsubsection{Selectivity}

Selectivity of a method is the measure of a method's ability to selectively detect the analyte from interferences present in the sample matrix. This is achieved through analyzing a sample containing the analyte and a sample containing only the sample matrix (the sample blank). A lack of response or detection in the sample matrix indicates a method's selectivity for the analyte. A sample of 1:1 $\mathrm{M}$ ratio sodium caseinate:caffeine and 1:1 $\mathrm{M}$ ratio sodium caseinate:5-CGA was analyzed along with the sodium caseinate control sample (the sample blank) containing only the sample matrix. Selectivity of the method was determined through a visual comparison of the chromatograms. The method correctly identified and quantified caffeine (Figure 4.10) and 5-CGA (Figure 4.11) in each sample but did not detect any peaks in the sample matrix at $280 \mathrm{~nm}$ or $330 \mathrm{~nm}$ (Figure 4.12 \& Figure 4.13). This indicates the developed method is 
selective both for caffeine and 5-CGA in the presence of a sodium caseinate matrix.

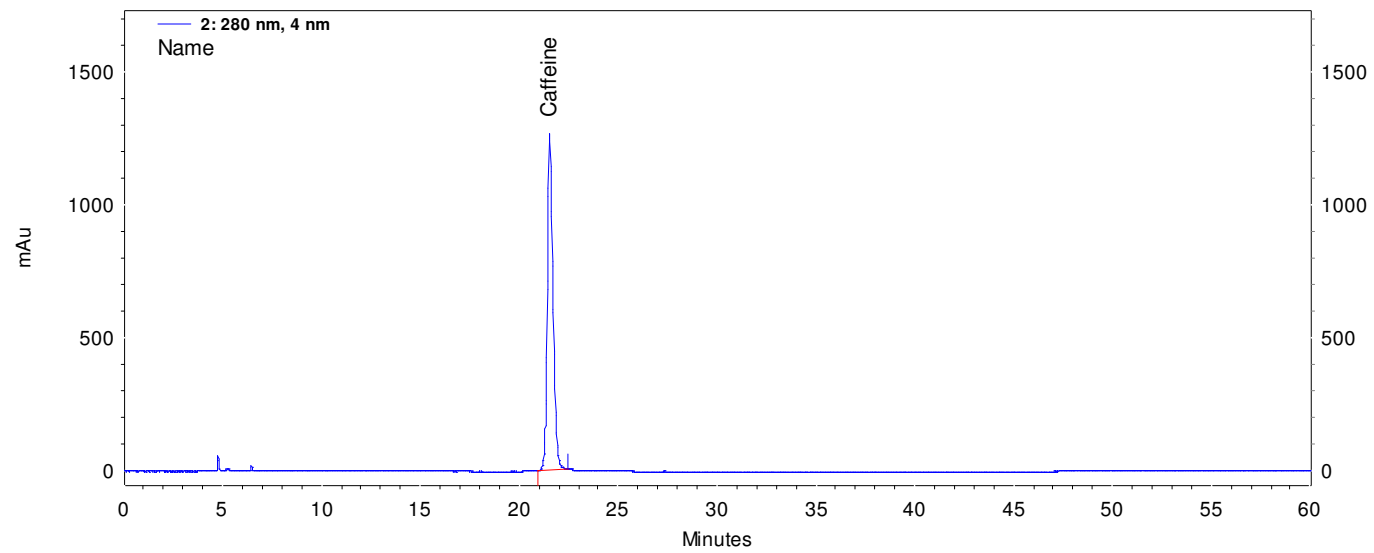

Figure 4.10. Sample chromatogram of 1:1 M ratio sodium caseinate:caffeine. Method correctly identified caffeine peak at $R T=21.5 \mathrm{~min}$.

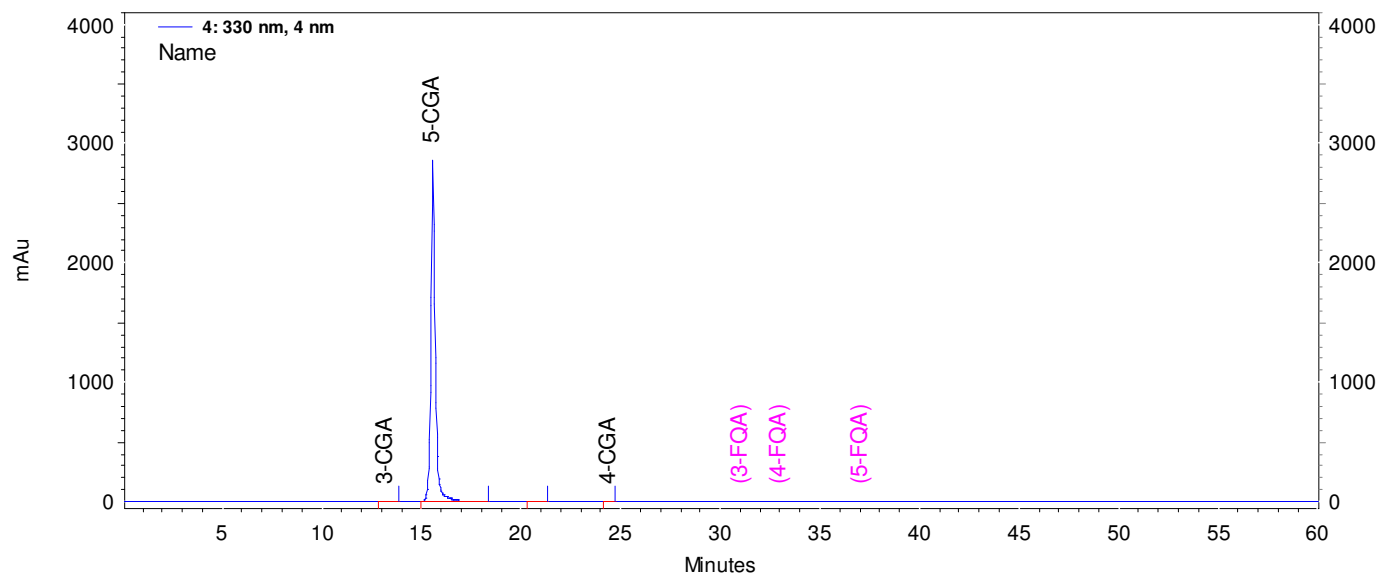

Figure 4.11. Sample chromatogram of 1:1 M ratio sodium caseinate:5-CGA. Note: peaks 3-CGA (RT 13.3 $\mathrm{min})$ and 4-CGA (RT 24.5 min) are known degradation products of 5-CGA. Undetected peaks are labeled in parentheses at their retention times. Method correctly identified 5-CGA peak at RT $=15.5 \mathrm{~min}$. 


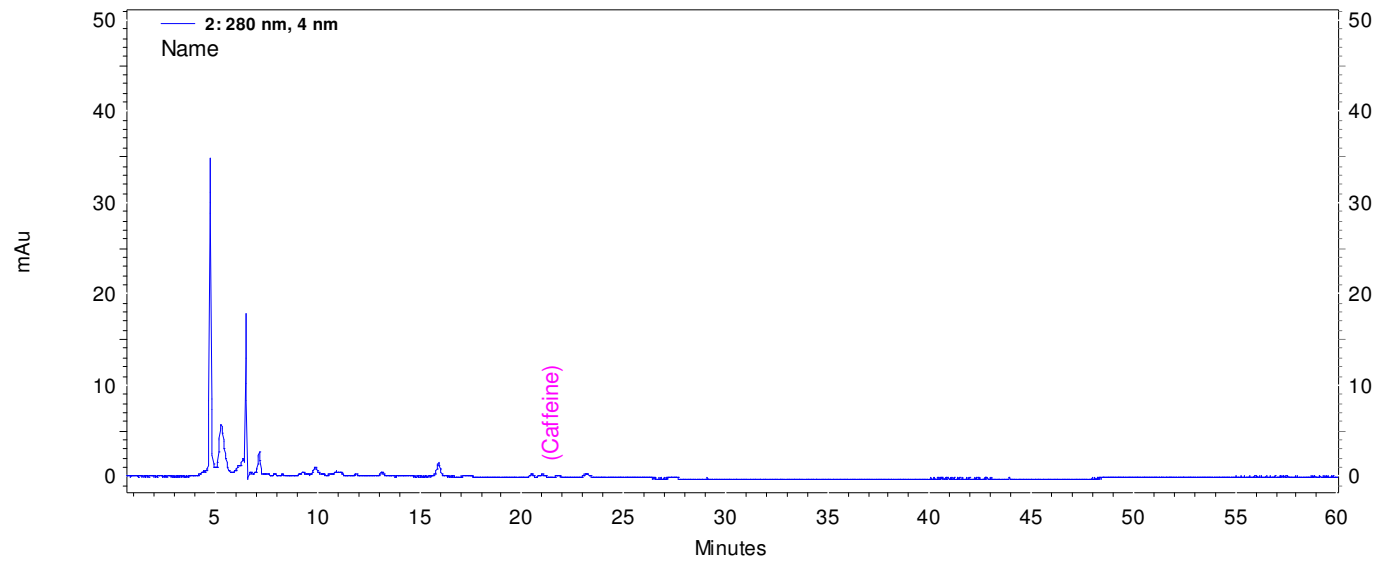

Figure 4.12. Sodium caseinate matrix (sample blank) measured at $280 \mathrm{~nm}$. Undetected peaks are labeled with parenthesis at their retention time.

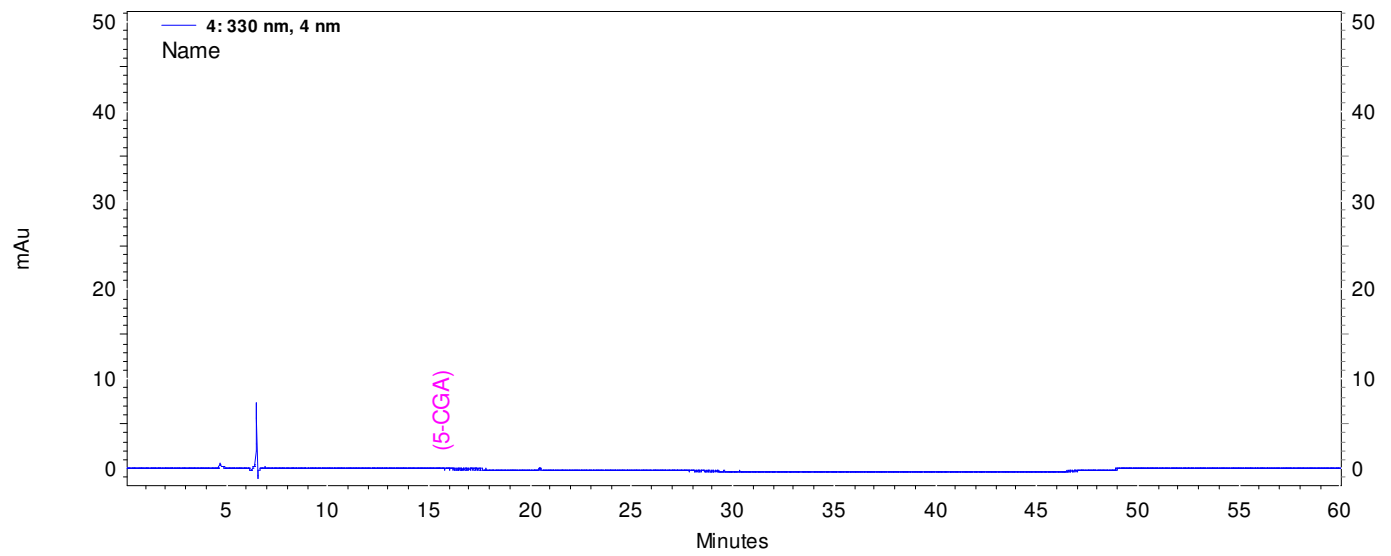

Figure 4.13. Sodium caseinate matrix (sample blank) measured at $330 \mathrm{~nm}$. Undetected peaks are labeled with parenthesis at their retention time.

\subsection{Experimental Design}

A Generalized Randomized Complete Block Design (GRCBD), blocked by day, was used as the design structure for these experiments. Blocked designs are used to control a nuisance factor; a known extraneous source of variation other than the treatment factors that affect the response. In these experiments, the day the experiments were performed was expected to have some effect on the response, therefore it was chosen as the random blocking factor. Complete block designs contain each treatment randomly assigned to at least one experimental 
unit in each block, and do not have any replication within blocks. A GRCBD was employed because all samples were prepared in duplicate, therefore generalized RCBDs allow for replication within blocks. RCBDs reduce variability of treatment comparisons by allowing them to be made on more similar units with a block, thus ensuring more accurate results. The GRCBD model equation is shown in Equation 4.1.

Equation 4.1 GRCBD model equation

$$
y_{i j k l}=\mu+\alpha_{i}+\beta_{j}+(\alpha \beta)_{i j}+\gamma_{k}+\varepsilon_{i j k l}
$$

Where: $\quad y_{i j k l}=$ the quantitative response, concentration of bioactive compound at the $i^{\text {th }}$ and $j^{\text {th }}$ treatment levels, $k^{\text {th }}$ block and $l^{\text {th }}$ replicate

$\mu=$ mean concentration of bioactive compound $\alpha_{i}=$ main effect of the $i^{\text {th }} \mathrm{pH}$ treatment level $\beta_{j}=$ main effect of the $j^{\text {th }}$ temperature treatment level $(\alpha \beta)_{i j}=$ interaction effect of the $i^{\text {th }} \mathrm{pH}$ and $j^{\text {th }}$ temperature treatment levels $\gamma_{k}=$ block effect from the $k^{\text {th }}$ block $\varepsilon_{i j k l}=$ random error term, we assume $\varepsilon_{i j k l} \sim \operatorname{IN}\left(0, \sigma^{2}\right)$

A two-series $2^{k}$ factorial design with $k$ factor level combinations is often employed during screening experiments. Screening experiments attempt to identify which factors, out of $k$ factors, significantly affect the response. Generally, levels of a factor are considered "low" and "high" to observe effects of the treatments on the response using extreme values in both directions. If the 
objective of the investigator is to optimize treatment conditions that significantly affect the response, more experiments can be redesigned holding insignificant factors constant while varying significant factors at more levels. The model equation for a two-series $2^{k}$ design is shown in Equation 4.2. For $2^{k}$ designs, each model term has 1 degree of freedom (df). The df for treatment factors used in these experiments are shown in Table 4.2.

Equation 4.2 Model equation for a two-series $2^{k}$ factorial design

$$
y_{i j k}=\mu+\alpha_{i}+\beta_{j}+(\alpha \beta)_{i j}+\varepsilon_{i j k}
$$

Where: $\quad y_{i j k}=$ the quantitative response, concentration of bioactive compound at the $i^{\text {th }}$ and $j^{\text {th }}$ treatment levels and $k^{\text {th }}$ replicate $\mu=$ mean concentration of bioactive compound $\alpha_{i}=$ main effect of the $i^{\text {th }} \mathrm{pH}$ treatment level $\beta_{j}=$ main effect of the $j^{\text {th }}$ temperature treatment level $(\alpha \beta)_{i j}=$ interaction effect of the $i^{\text {th }} \mathrm{pH}$ and $j^{\text {th }}$ temperature treatment levels $\varepsilon_{i j k}=$ random error term, we assume $\varepsilon_{i j k} \sim \operatorname{IN}\left(0, \sigma^{2}\right)$

Table 4.2. ANOVA table and df of treatment levels

\begin{tabular}{lc}
\hline Source & df \\
\hline $\mathrm{pH}$ & 1 \\
Temperature & 1 \\
$\mathrm{pH}^{*}$ Temperature & 1 \\
Block (day) & 1 \\
Error & 11 \\
Total & 15 \\
\hline
\end{tabular}




\subsection{Binding caffeine \& 5-CGA to sodium caseinate}

\subsubsection{Binding caffeine to sodium caseinate trials}

To estimate the amount of binding that occurred between sodium caseinate and caffeine, a sample of the supernatant from each treatment combination was analyzed by RP-HPLC on the same day they were prepared. The concentration of caffeine recovered in the supernatant of each sample was calculated using a caffeine calibration curve, constructed prior to the sample analysis using a least squares regression line (Figure 4.14). The final caffeine concentration detected in the supernatant of each sample is shown in Figure 4.15. The concentration of caffeine was then converted to the percent of caffeine recovered from the known amount that was added to each sample, with the caffeine control sample containing $100 \%$ recovery of the added caffeine. The percentage of caffeine that was bound to sodium caseinate was then estimated based on the calculated difference between the initial amount of caffeine added and the amount of that was recovered in the supernatant. Therefore, the lesser amount of caffeine detected in the supernatant suggests more binding had occurred with the sodium caseinate. 


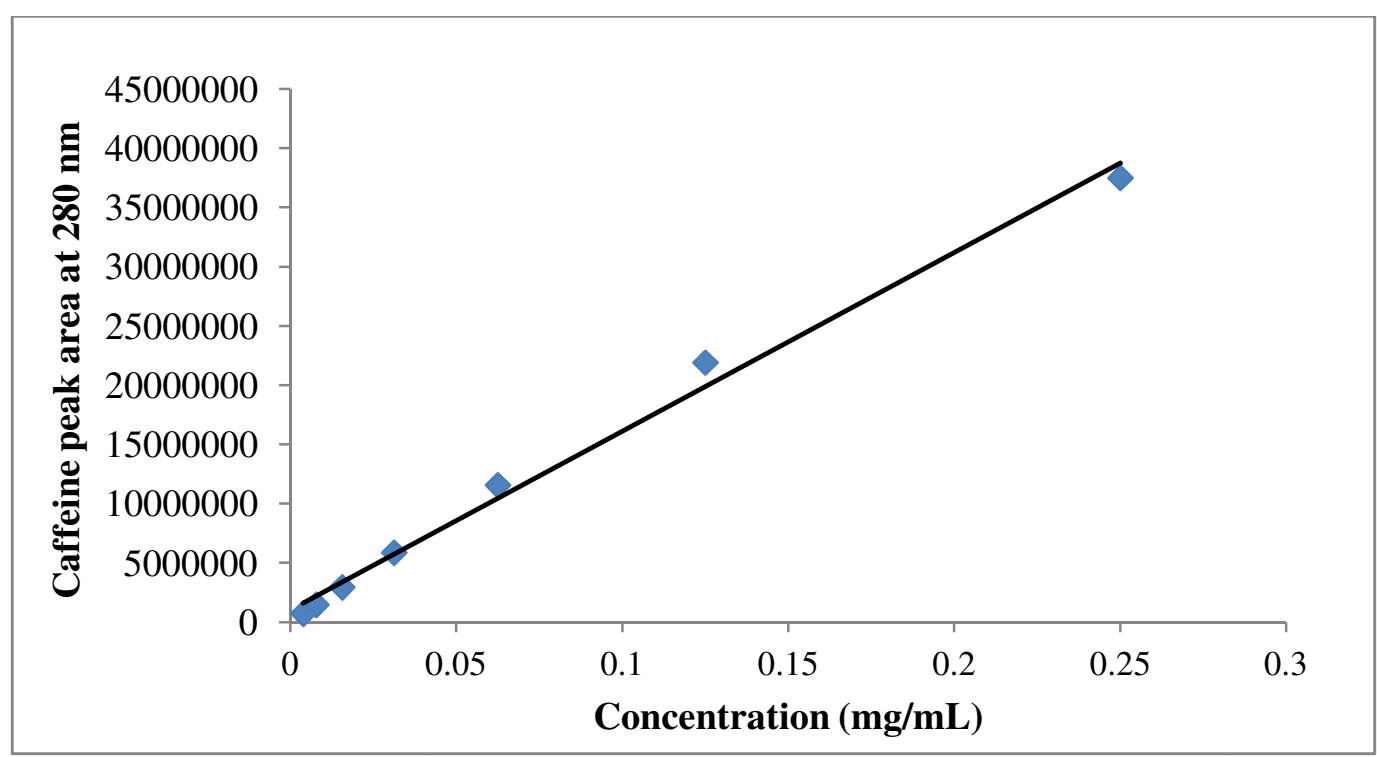

Figure 4.14. Caffeine calibration curve of peak area (mAU) vs. concentration $(\mathrm{mg} / \mathrm{mL}) \cdot R^{2}=0.9924$.

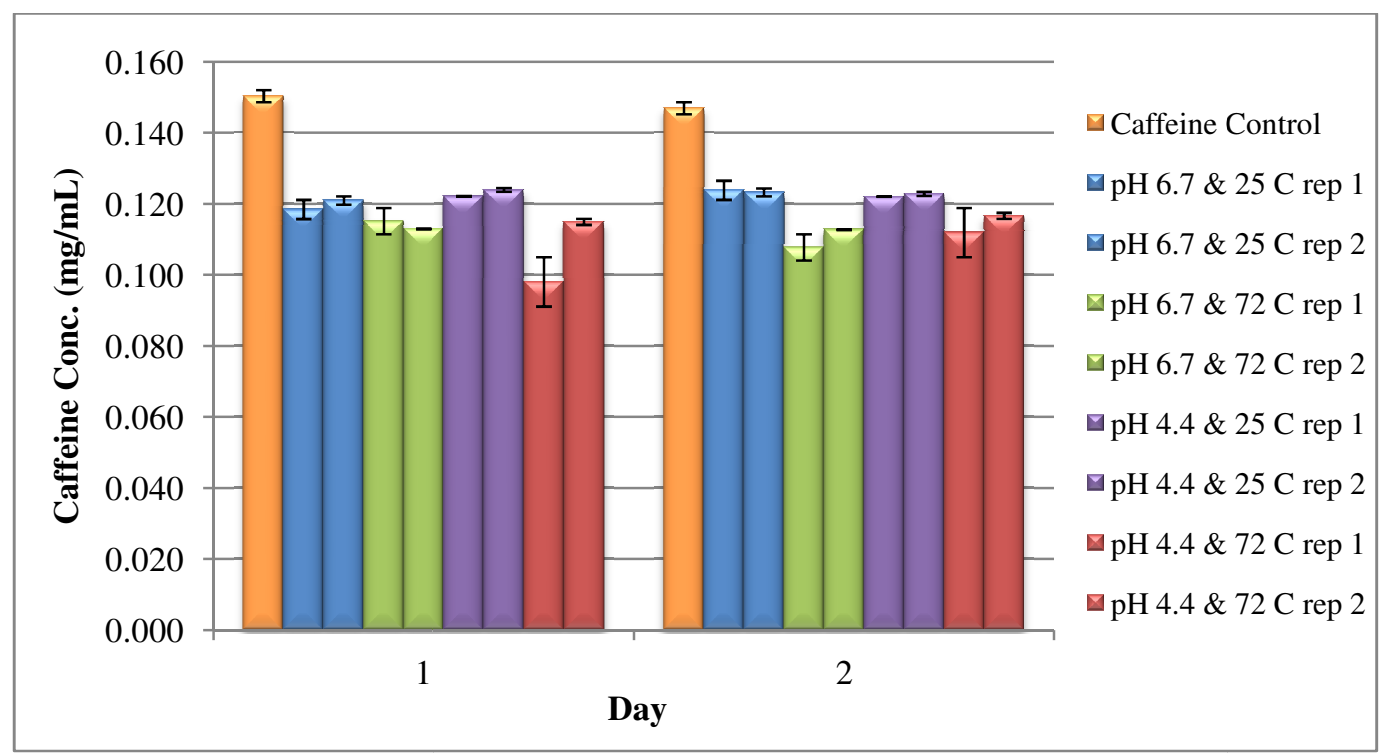

Figure 4.15. Concentration of caffeine detected in the supernatant of all samples prepared on Day 1 and Day 2. Caffeine control represents a caffeine standard solution with no sodium caseinate. Error bars represent standard error of each sample.

The concentration of caffeine detected in the supernatant of each sample was analyzed with an overall significance level at $\alpha_{\mathrm{E}}=0.05$. A Bonferroni adjustment was performed to control Experimentwise (EW) error rate, thereby decreasing the rate of Type 1 error and resulting in an individual significance Page 70 
level of $\alpha_{\mathrm{I}}=0.0167$. There was not enough evidence to conclude that there was any significant interaction effect of $\mathrm{pH}$ and temperature on the true mean concentration of caffeine recovered in the samples $(p=0.5597)$. Since there was no significant interaction effect ( $\mathrm{p}>0.01)$, interpretation of the main effects of $\mathrm{pH}$ and temperature may proceed. There was also not enough evidence to conclude that the main effect of $\mathrm{pH}$ had a significant effect on the true mean concentration of caffeine recovered in the samples $(\mathrm{p}=0.8983)$, however the main effect of temperature did have a significant effect on the true mean concentration of caffeine recovered in the samples $(\mathrm{p}=0.0008)$. Samples prepared at $72{ }^{\circ} \mathrm{C}$ contained significantly less caffeine than samples prepared at $25^{\circ} \mathrm{C}$, an average of $0.011 \mathrm{mg} / \mathrm{mL}$ less which corresponds to a calculated percentage of approximately $9 \%$ more caffeine bound at $72{ }^{\circ} \mathrm{C}$ than at $25^{\circ} \mathrm{C}$ (Figure 4.16 ).

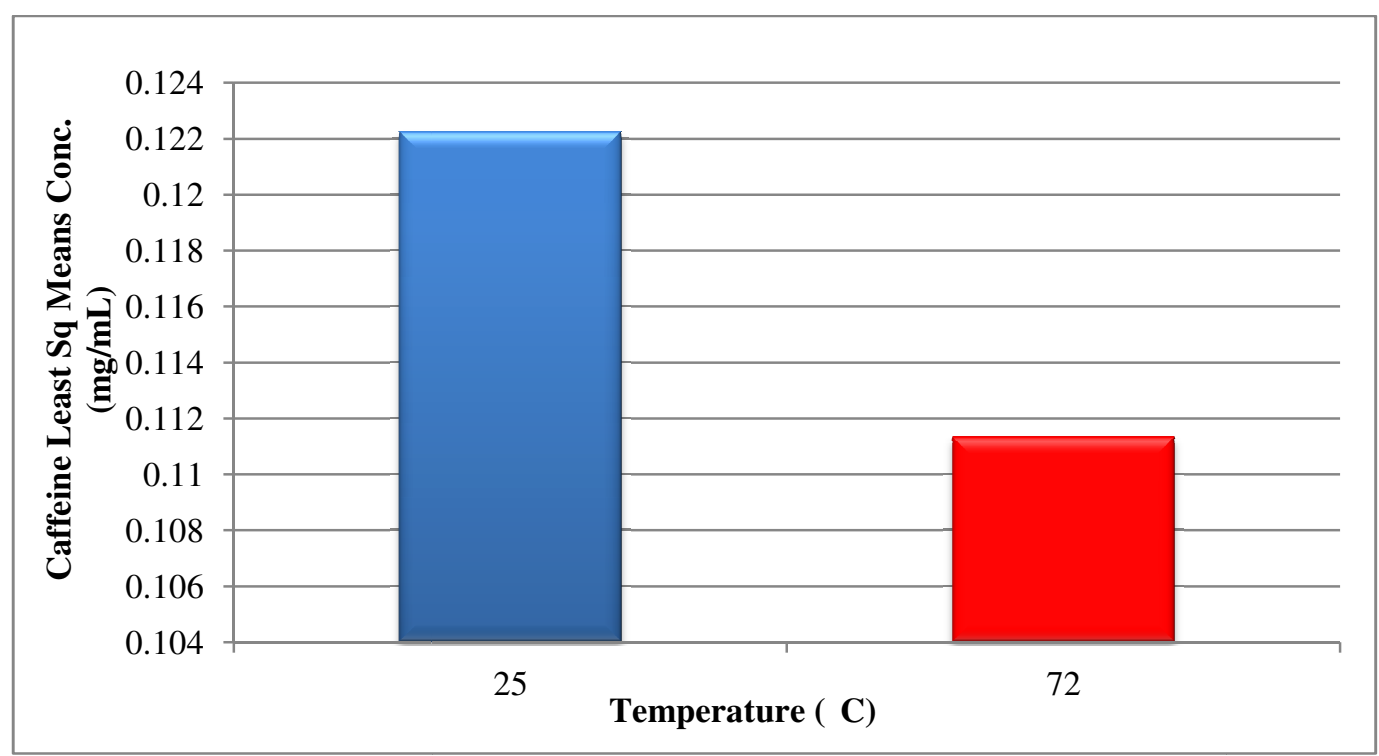

Figure 4.16. Least square means of caffeine concentration in supernatant of samples prepared at $25^{\circ} \mathrm{C}$ and $72{ }^{\circ} \mathrm{C}$.

With $95 \%$ overall confidence, the effect of temperature at $72{ }^{\circ} \mathrm{C}$ on the mean caffeine concentration is between $0.00418 \mathrm{mg} / \mathrm{mL}$ and $0.01765 \mathrm{mg} / \mathrm{mL}$ less 
than the effect of temperature at $25^{\circ} \mathrm{C}$ on the mean caffeine concentration detected in the sample supernatant (Appendix B). The percent recovery of caffeine in samples prepared at $72{ }^{\circ} \mathrm{C}$ ranged between $65-80 \%$, whereas samples prepared at $25^{\circ} \mathrm{C}$ had a percent caffeine recovery ranging from $79-84 \%$. Therefore, a calculated percentage of approximately $20-35 \%$ of the added caffeine was estimated to have been bound to sodium caseinate in samples prepared at $72{ }^{\circ} \mathrm{C}$. This corresponds to a concentration of caffeine between $0.0303-0.0523 \mathrm{mg} / \mathrm{mL}$ with a calculated average of $0.0413 \mathrm{mg} / \mathrm{mL}$ caffeine bound to sodium caseinate. This is compared to a calculated percentage of approximately $16-21 \%$ caffeine bound in samples prepared at $25^{\circ} \mathrm{C}$. This corresponds to a concentration of caffeine bound to sodium caseinate between $0.0231-0.0319 \mathrm{mg} / \mathrm{mL}$ with a calculated average of $0.0275 \mathrm{mg} / \mathrm{mL}$ caffeine bound to sodium caseinate at $25^{\circ} \mathrm{C}$ (Figure 4.17). Therefore, it was determined that $72{ }^{\circ} \mathrm{C}$ was the best of the two temperatures investigated that had a significant effect on the amount of caffeine recovered in the sample supernatant and thus also had a significant effect on the extent of binding between caffeine and sodium caseinate. The data was also analyzed for any violations of the ANOVA model assumptions (normality, equal variances). Because the experimental design was balanced, no violations of the model assumptions were detected (Appendix D). 


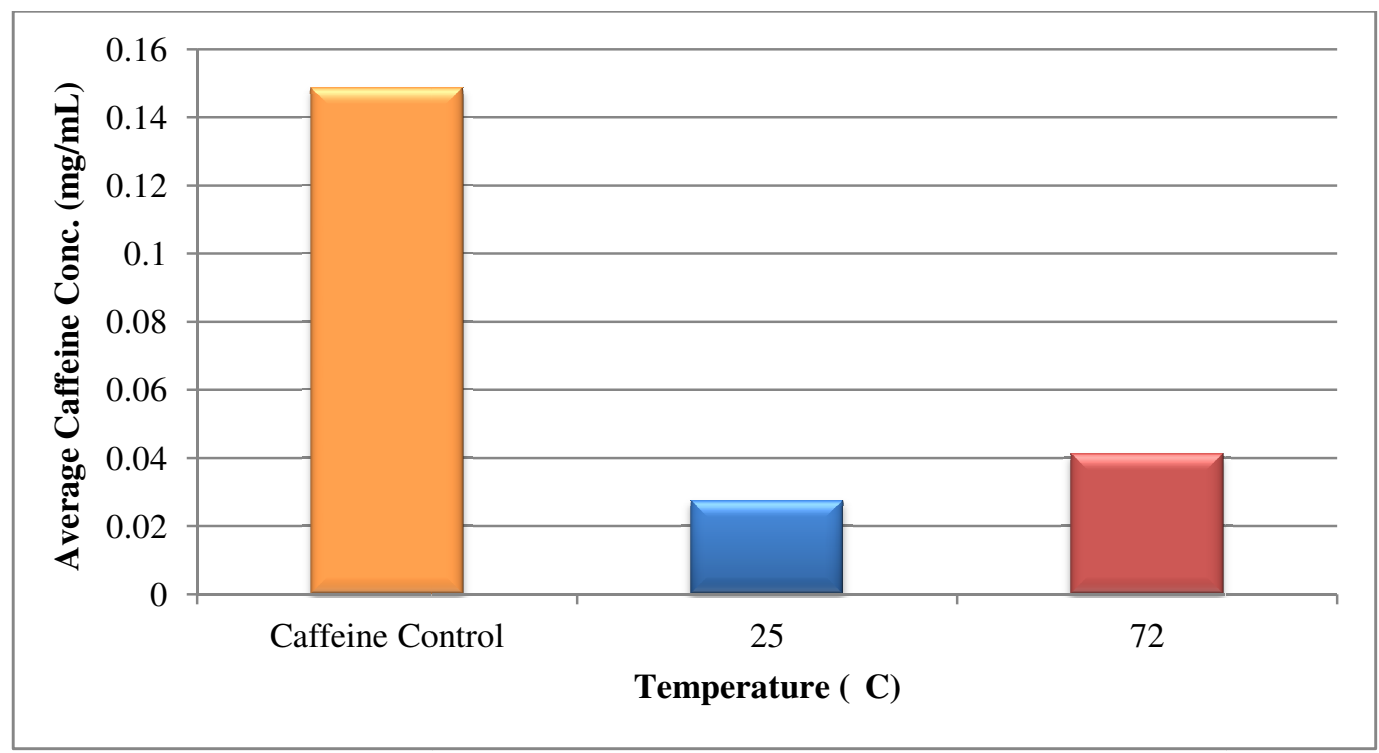

Figure 4.17. Calculated average concentration of caffeine bound to sodium caseinate at $25^{\circ} \mathrm{C}$ and $72{ }^{\circ} \mathrm{C}$. Caffeine control represents the average theoretical amount of caffeine bound to sodium caseinate at $100 \%$.

Temperature was the only treatment that had a significant effect on the concentration of caffeine detected in the samples. The results from these experiments have also shown that optimal binding interactions between caffeine and sodium caseinate is temperature dependent, with a higher temperature resulting in greater binding. This suggests that the most likely mechanism for binding caffeine to sodium caseinate is via hydrophobic interactions. Hydrophobic interactions are highly temperature dependent, and because $\mathrm{pH}$ was not observed to have a significant effect on caffeine concentration, the binding mechanism was not likely due to electrostatic interactions. Therefore, conformational changes to the casein structure, such as denaturation of the protein at high temperatures, may increase binding sites available to caffeine. 


\subsubsection{Binding 5-CGA to sodium caseinate trials}

The estimated amount of 5-CGA that was bound to sodium caseinate was measured in the same way as previously described with caffeine samples. A calibration curve of 5-CGA was also constructed prior to any sample analysis and used to calculate the concentration of 5-CGA detected in sample supernatants (Figure 4.18). The final 5-CGA concentration detected in the supernatant of each sample is shown in Figure 4.19.

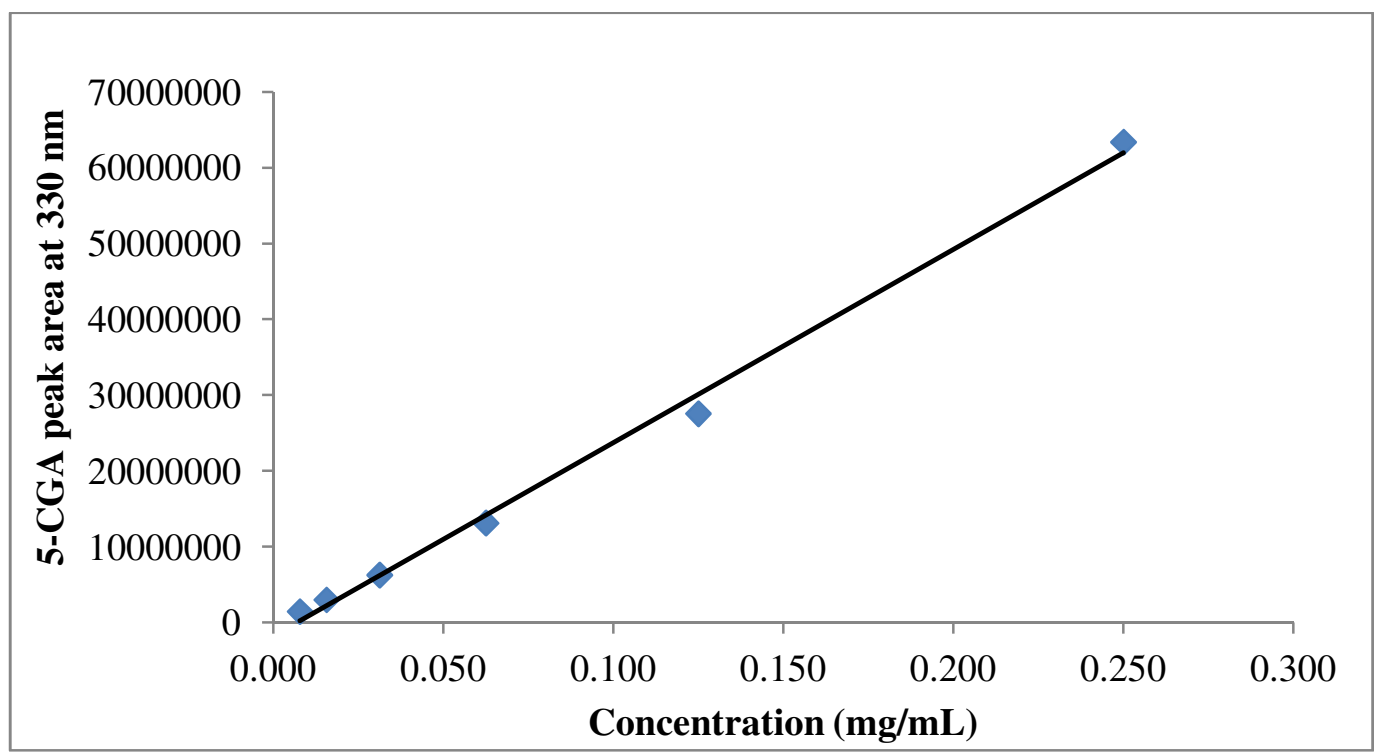

Figure 4.18. 5-CGA calibration curve of peak area (mAU) vs. concentration $(\mathrm{mg} / \mathrm{mL})$ at $330 \mathrm{~nm} . R^{2}=0.9958$. 


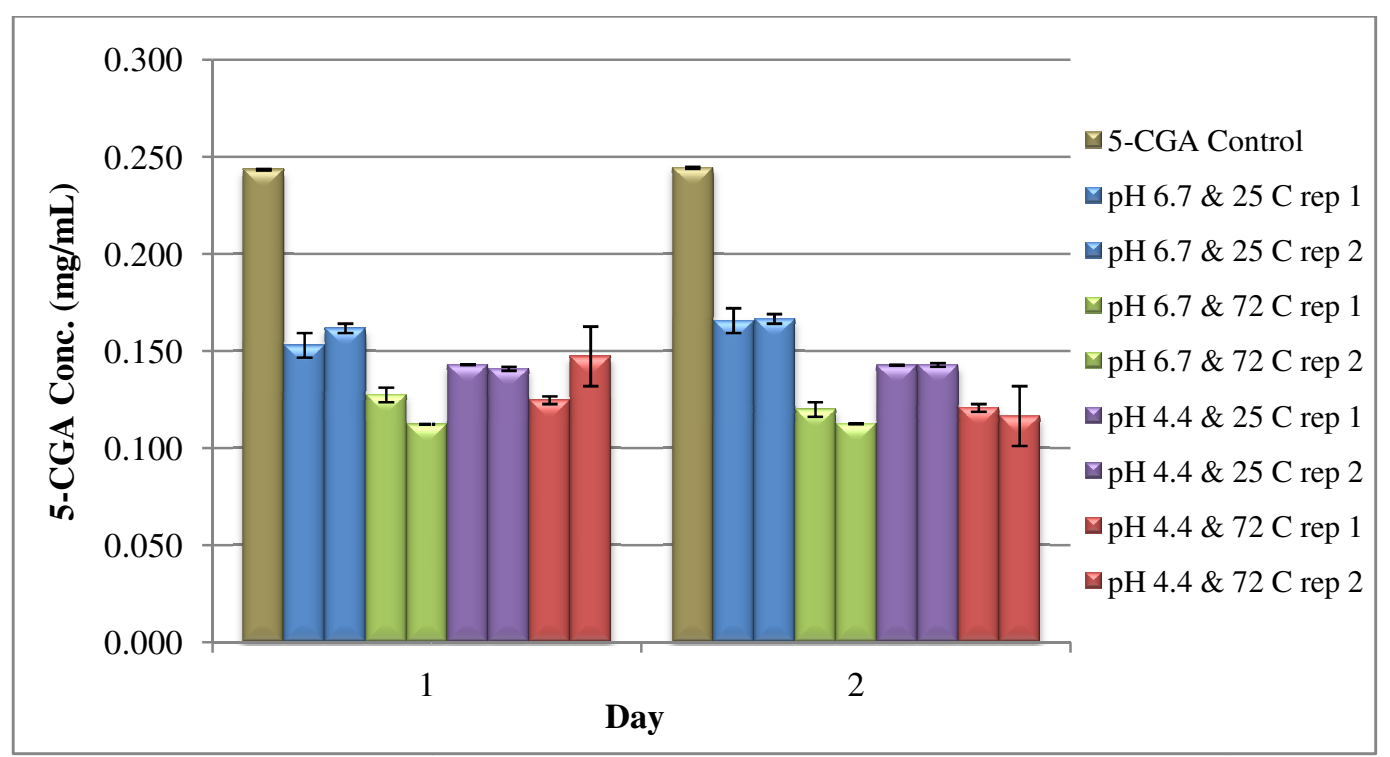

Figure 4.19. Concentration of 5-CGA detected in the supernatant of all samples prepared on Day 1 and Day 2. 5-CGA control represents a 5-CGA standard solution with no sodium caseinate. Error bars represent standard error of each sample.

At an individual significance level of $\alpha_{\mathrm{I}}=0.0167$, there was enough evidence to conclude that there was a significant interaction effect of $\mathrm{pH}$ and temperature on the true mean 5-CGA concentration detected in the supernatant of samples $(\mathrm{p}=0.0066)$. Though the main effect of temperature also had a significant effect on the true mean 5-CGA concentration $(\mathrm{p}<0.0001)$, main effects should not be interpreted if interaction effects are significant. Therefore, the remainder of the statistical analysis will only focus on the interaction effect of $\mathrm{pH}$ and temperature. There was not enough evidence to conclude that the main effect of $\mathrm{pH}$ had a significant effect on the true mean 5-CGA concentration detected in the sample supernatant $(\mathrm{p}=0.2631)$.

A post-hoc analysis, Tukey’s Honestly Significant Difference (HSD) pairwise comparison was performed after completion of the ANOVA statistical analysis to determine which treatment combinations of $\mathrm{pH}$ and temperature 
resulted in significantly different and increased binding interactions of 5-CGA to sodium caseinate (Appendix C). The treatment combinations pH 6.7 and $72{ }^{\circ} \mathrm{C}$ resulted in the lowest average 5-CGA concentration detected in the sample supernatant and was thus the best of the investigated conditions for binding 5CGA to sodium caseinate. The percentage of 5-CGA recovered in the sample supernatants at $\mathrm{pH} 6.7$ and $72{ }^{\circ} \mathrm{C}$ ranged between $46-52 \%$, therefore the calculated percentage of 5-CGA bound to sodium caseinate was estimated to be between $48-54 \%$ of the original amount of 5-CGA added. This corresponds to a calculated concentration of 5-CGA bound to sodium caseinate between 0.1159 $0.1311 \mathrm{mg} / \mathrm{mL}$ with a calculated average of $0.1235 \mathrm{mg} / \mathrm{mL} 5$-CGA bound. Samples treated at $\mathrm{pH} 6.7$ and $25^{\circ} \mathrm{C}$ had a calculated concentration of 5-CGA bound to sodium caseinate that ranged between $0.0779-0.0903 \mathrm{mg} / \mathrm{mL}$ resulting in a calculated average of $0.0841 \mathrm{mg} / \mathrm{mL} 5$-CGA bound (Figure 4.20). Compared to samples treated at $\mathrm{pH} 6.7$ and $25^{\circ} \mathrm{C}$ which resulted in a 5-CGA percent recovery ranging from $62-68 \%$, the treatment conditions at $\mathrm{pH} 6.7$ and $72{ }^{\circ} \mathrm{C}$ resulted in a calculated average increase of approximately $16.5 \%$, or approximately $0.0437 \mathrm{mg} / \mathrm{mL}$ more 5 -CGA bound to sodium caseinate. This data was also analyzed for any violations of the ANOVA model assumptions (normality, equal variances). Because the experimental design was balanced, no violations of the model assumptions were detected. (Appendix E). 


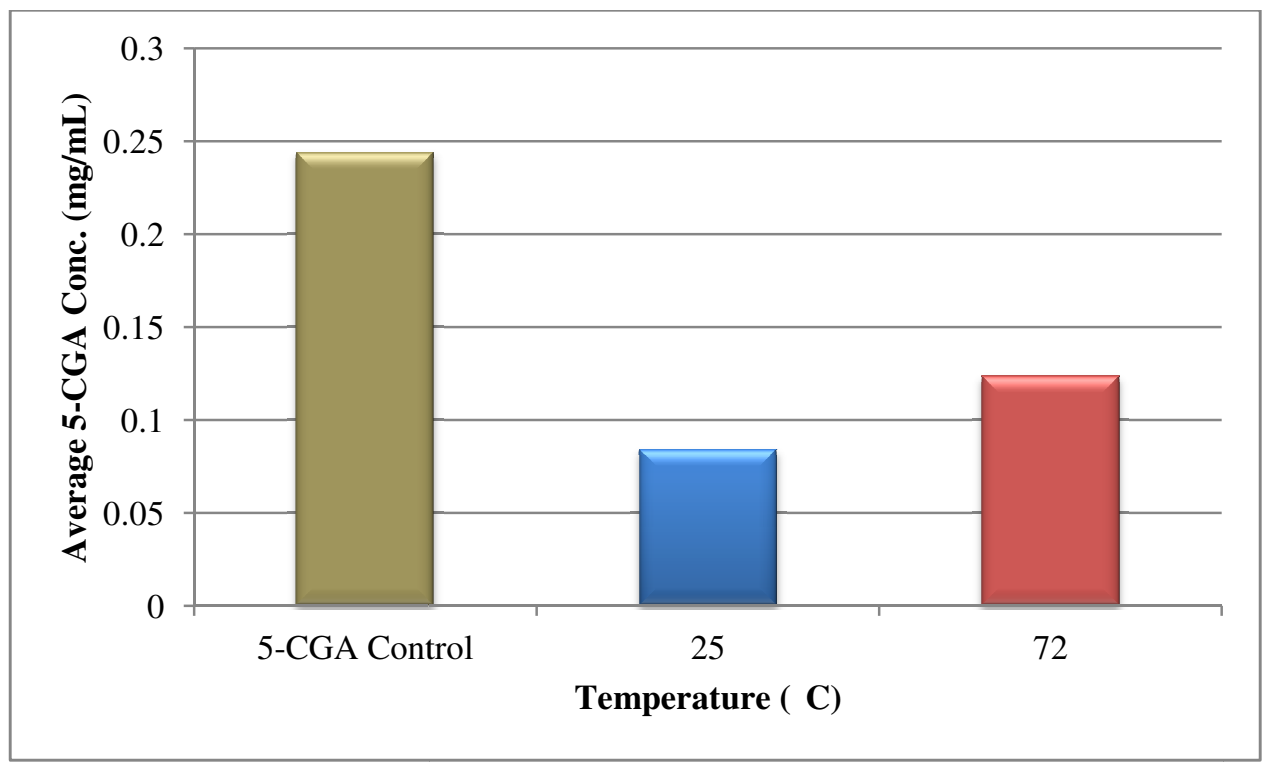

Figure 4.20. Calculated average concentration of 5-CGA bound to sodium caseinate at $25^{\circ} \mathrm{C}$ and $72{ }^{\circ} \mathrm{C}$, each at $\mathrm{pH}$ 6.7. 5-CGA control represents the average theoretical amount of 5-CGA bound to sodium caseinate at 100\%.

The results from these experiments show that the treatment combination of $\mathrm{pH}$ at 6.7 and temperature at $72{ }^{\circ} \mathrm{C}$ provided the best treatment combination for binding 5-CGA to sodium caseinate of all treatments investigated. This suggests that casein with a net negative charge at $\mathrm{pH} 6.7$ and some extent of protein denaturation at $72{ }^{\circ} \mathrm{C}$ may increase binding sites available for 5-CGA. Both hydrophobic and electrostatic interactions are likely to be the mechanism of binding for 5-CGA to sodium caseinate as both $\mathrm{pH}$ and temperature had some influence on binding behavior.

\subsubsection{Binding caffeine \& 5-CGA present in green coffee extract to sodium}

\section{caseinate trials}

After determining the optimal conditions of the investigated treatments for binding pure standards of caffeine and 5-CGA to sodium caseinate, we were interested to see whether these conditions would result in a similar extent of 
binding from a sample of green coffee extract that naturally contained both these compounds. The green coffee extract also contained numerous other unidentified compounds, thus this trial would demonstrate any interferences or competition of binding to sodium caseinate.

Immediately upon addition of the green coffee extract to the sodium caseinate solution, the color of the solution turned from opaque white to dark gray-green. The color of the solution continued to develop while being stirred for $1 \mathrm{~h}$, turning into a dark emerald green color (Figure 4.21). During isoelectric precipitation with $0.1 \mathrm{~N} \mathrm{HCl}$, the sample solution returned to a bright white color and once the isoelectric point was reached $(\mathrm{pH} 4.6)$ the supernatant turned to a clear, light green color (Figure 4.22). The precipitated caseins contained a greenish hue in comparison to its usual bright white color. This indicated that some compounds responsible for the color of green coffee had been bound to sodium caseinate, possibly including 5-CGA as samples containing pure 5-CGA in previous studies resulted in a similar color change. Once the green coffee casein pellet was dried and compared to a control casein pellet, a noticeable green color remained in the green coffee pellet while no color change was observed in the control (Figure 4.23). 


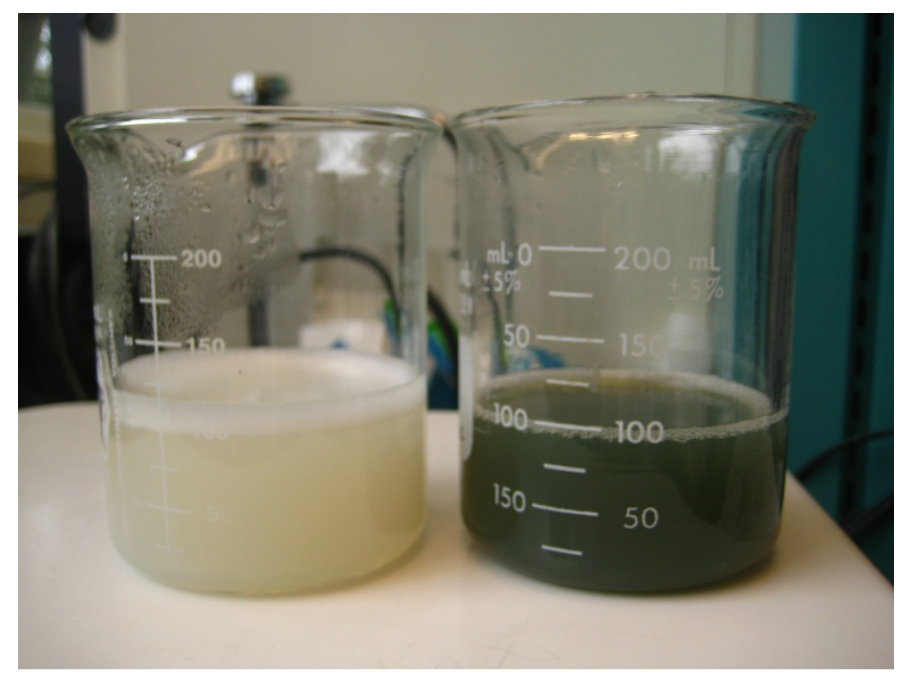

Figure 4.21. Sodium caseinate solution before addition of green coffee extract (left) and after addition of green coffee extract (right).

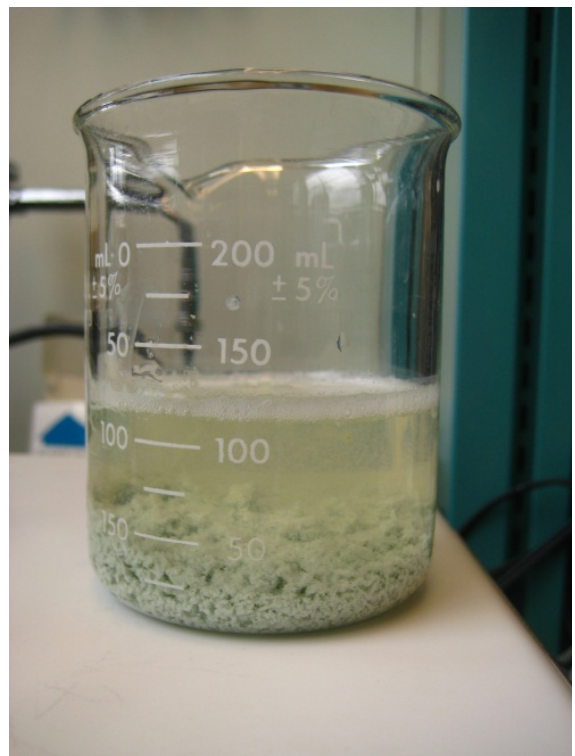

Figure 4.22. Sample of sodium caseinate bound with green coffee extract after isoelectric precipitation at $\mathrm{pH} 4.6$ and $30^{\circ} \mathrm{C}$. 


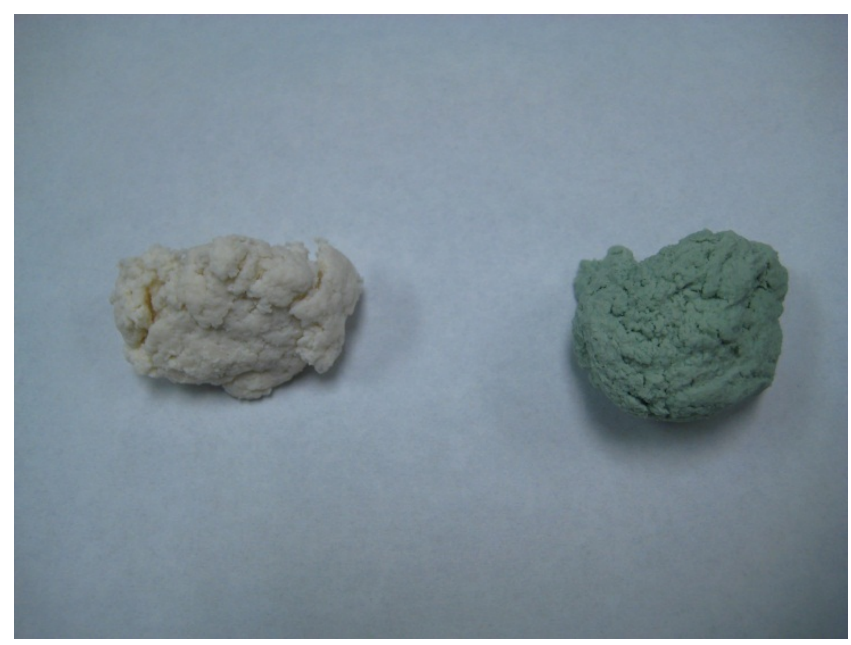

Figure 4.23. Casein pellets after centrifugation. Sodium caseinate control pellet (left) and sodium caseinate with green coffee extract pellet (right).

The concentration of caffeine and 5-CGA detected in the supernatant of sodium caseinate with green coffee extract samples was comparable to results using commercial standards. Each sample was prepared in triplicate (Figure 4.24 and Figure 4.25). The average percentage of caffeine bound to sodium caseinate was $16.51 \%( \pm 0.04)$ corresponding to a calculated average concentration of $0.1605 \mathrm{mg} / \mathrm{mL}$ caffeine bound from the total caffeine concentration of 0.9722 $\mathrm{mg} / \mathrm{mL}$ in the pure green coffee extract. The average percentage of CGA bound to sodium caseinate was $47.90 \%( \pm 0.04)$, corresponding to a calculated average concentration of $2.098 \mathrm{mg} / \mathrm{mL}$ CGA bound from the total CGA concentration of $4.379 \mathrm{mg} / \mathrm{mL}$ in the pure green coffee extract (Figure 4.26). These results confirmed the optimal binding conditions determined for each respective bioactive compound in previous studies using commercial standards. However, it is important to note that results from these experiments represent only an approximation, a calculated average concentration of the bioactive compound estimated to have been bound to sodium caseinate. Without direct measurement of 
the casein pellet for the concentration of bioactive compound actually bound, there is no way of definitively assessing the true concentration of the bound material.

There appeared to be no detectable interfering compounds in the green coffee extract that inhibited binding of caffeine or 5-CGA. A combination of hydrophobic and electrostatic interactions is also likely to be the binding mechanism between caffeine and 5-CGA to sodium caseinate from green coffee extracts.

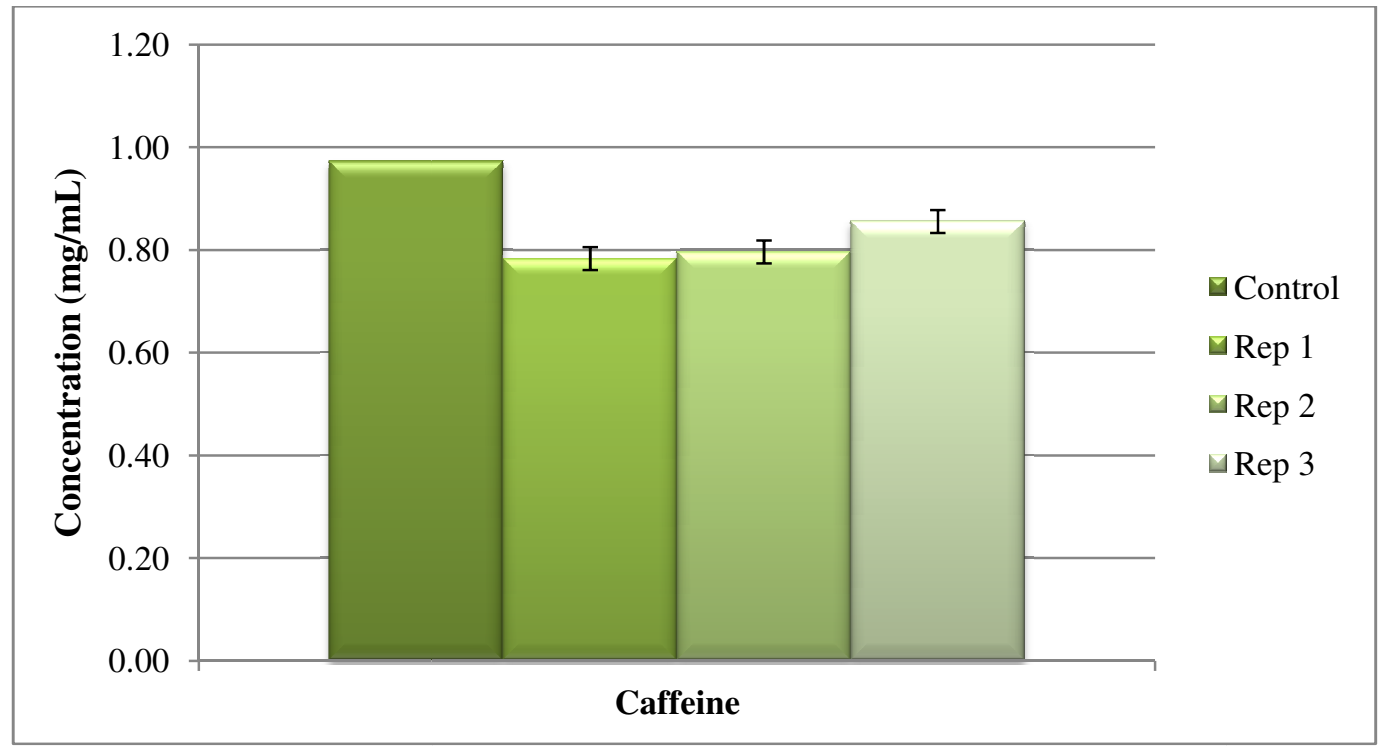

Figure 4.24. Concentration of caffeine detected in the supernatant of green coffee extract with sodium caseinate samples. Error bars represent standard deviation of each sample. 


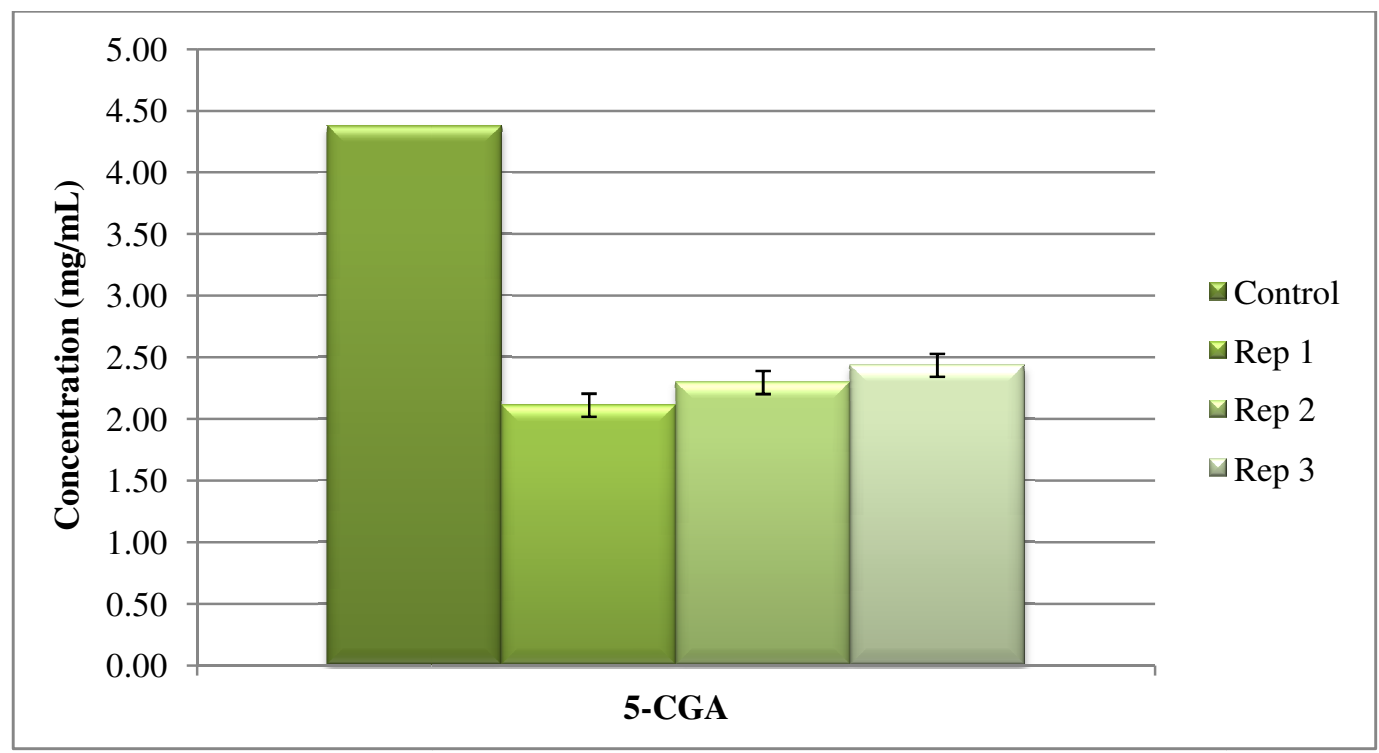

Figure 4.25. Concentration of 5-CGA detected in the supernatant of green coffee extract with sodium caseinate samples. Error bars represent standard deviation of each sample.

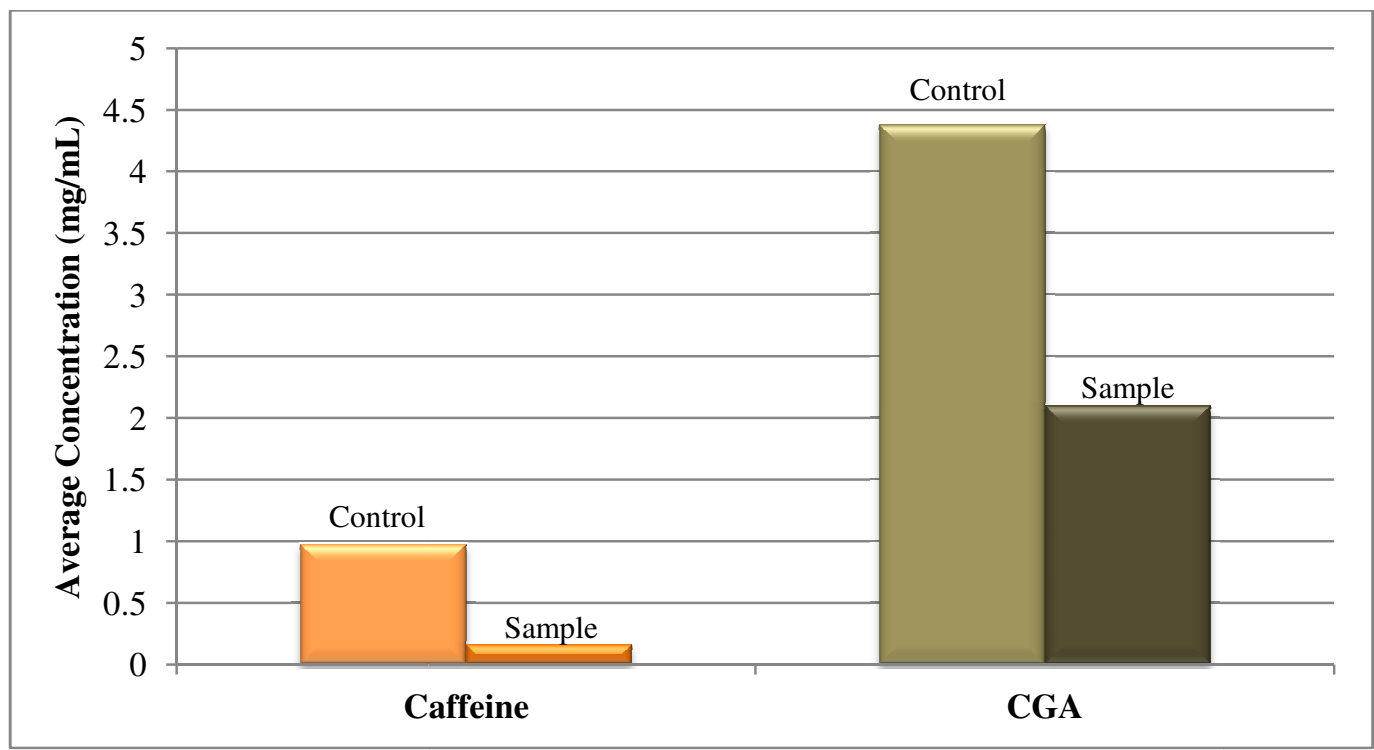

Figure 4.26. Calculated average concentration of caffeine and total CGA bound to sodium caseinate from green coffee extract at optimal treatment conditions $\mathrm{pH}$ $6.7 \& 72{ }^{\circ} \mathrm{C}$. Controls represent the amount of bioactive compound detected in a pure green coffee extract sample.

\subsection{HPLC trials}

The concentration of caffeine and 5-CGA bound to sodium caseinate was determined through calculating a difference between the added concentration of 
bioactive compound in a sample and the concentration recovered in the supernatant through HPLC analysis. A commercial standard of caffeine was injected and measured at $280 \mathrm{~nm}$ to determine the retention time of caffeine (Figure 4.27). Likewise, a commercial standard of 5-CGA was injected and measured at $330 \mathrm{~nm}$ to determine the retention time of 5-CGA (Figure 4.28). Green coffee extract samples contained CGA isomers with known retention times from previous studies. Retention times of all compounds are summarized in Table 4.3.

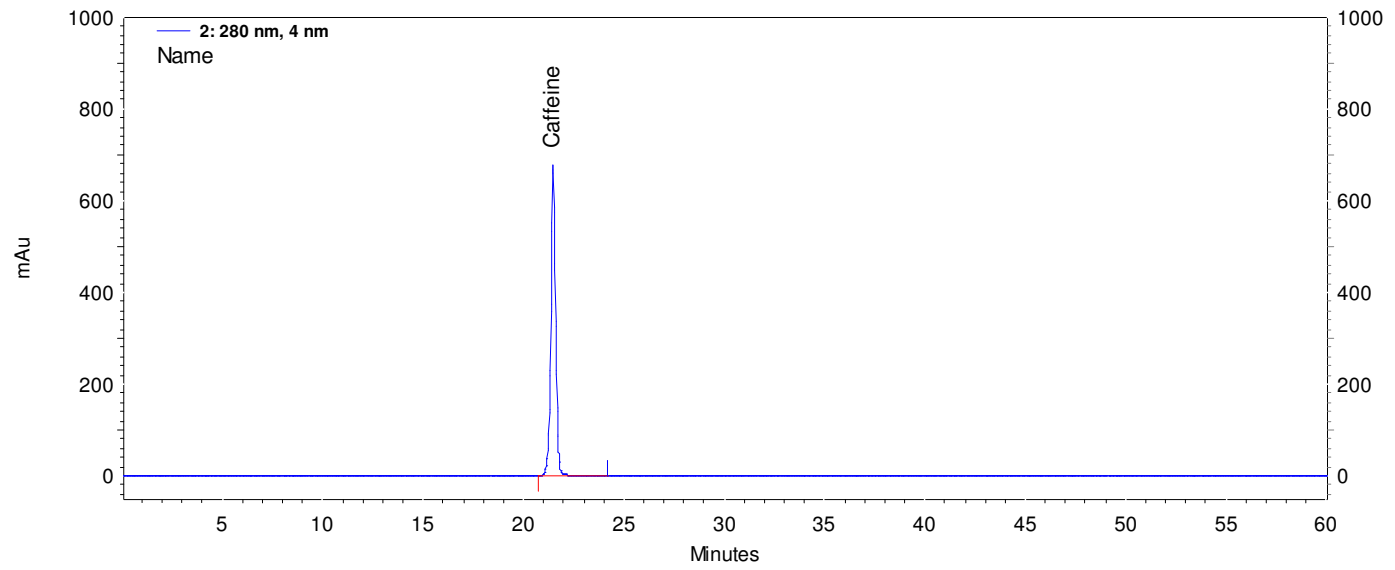

Figure 4.27. Caffeine standard (1:16 dilution) measured at $280 \mathrm{~nm}$.

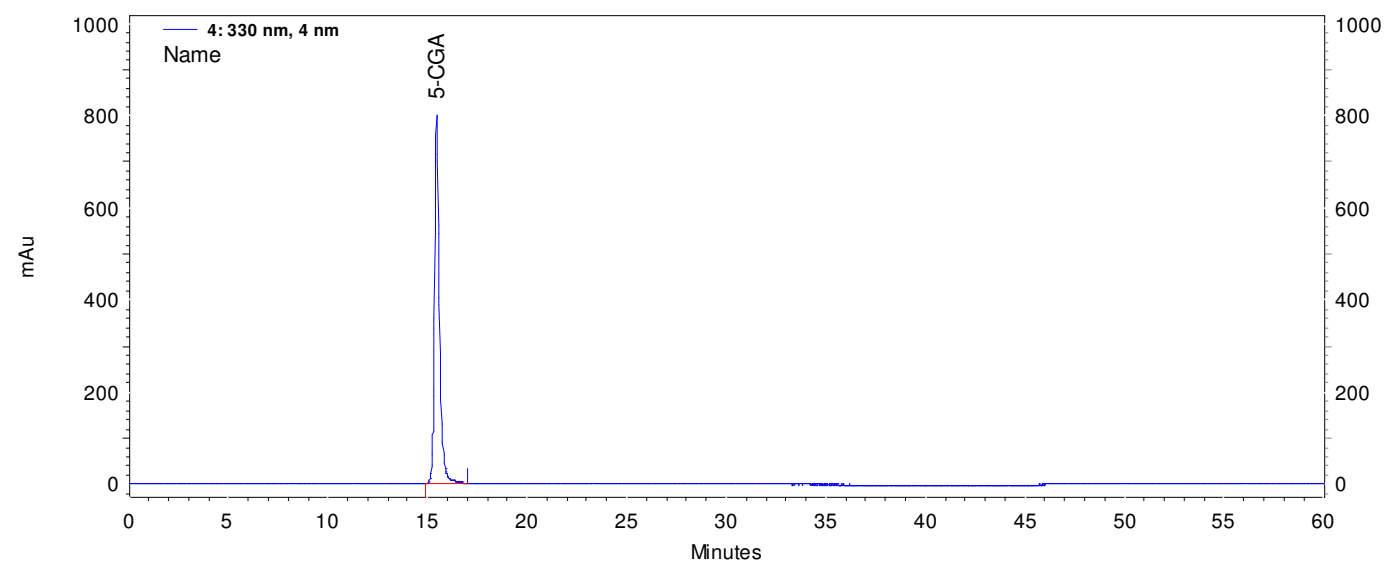

Figure 4.28. CGA standard (1:16 dilution) measured at $330 \mathrm{~nm}$. 
Table 4.3. Retention times and relative retention times of caffeine and CGA isomers

\begin{tabular}{ccc}
\hline Analyte & $\begin{array}{c}\text { Retention Time } \\
(\mathbf{m i n})\end{array}$ & $\begin{array}{c}\text { Relative Retention Time } \\
(\mathbf{m i n})\end{array}$ \\
\hline Caffeine & 21.4 & 1.000 \\
3-CGA & 13.2 & 0.617 \\
4-CGA & 23.2 & 1.080 \\
5-CGA & 15.5 & 1.000 \\
3-FQA & 29.4 & 1.900 \\
4-FQA & 30.9 & 1.990 \\
5-FQA & 34.7 & 2.240 \\
\hline
\end{tabular}

In sodium caseinate samples containing caffeine, a single caffeine peak was observed at approximately RT $=21.5 \mathrm{~min}$, with no other impurity or degradation peaks observed in the chromatogram (Figure 4.29). In sodium caseinate samples containing 5-CGA, a large 5-CGA peak was observed at approximately RT $=15.5$ min, along with two minor peaks constituting 3-CGA $(\mathrm{RT}=13.2 \mathrm{~min})$ and 4CGA $(\mathrm{RT}=23.2 \mathrm{~min})$ that are likely due to degradation of CGA upon heating (Figure 4.30). It is important to note that degradation compounds were mostly observed in samples treated at $72{ }^{\circ} \mathrm{C}$ which is consistent with the behavior of CGAs upon heating.

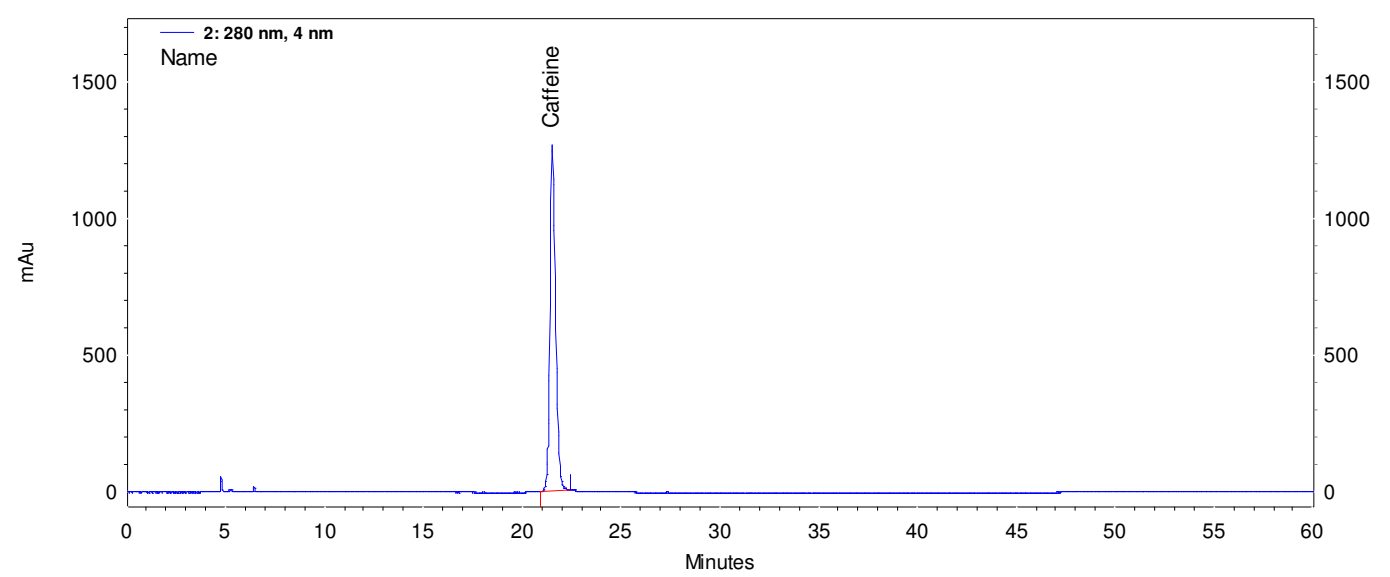

Figure 4.29. Sample chromatogram of 1:1 M sodium caseinate:caffeine sample (pH 6.7, $\left.72^{\circ} \mathrm{C}\right)$. 


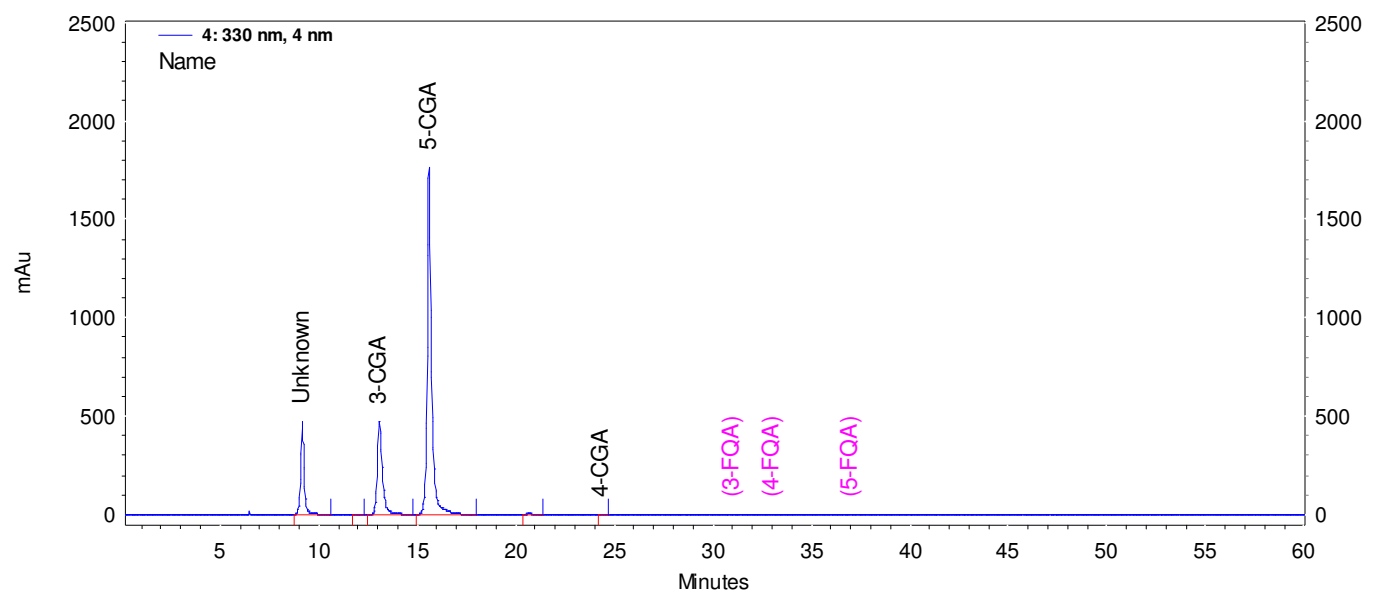

Figure 4.30. Sample chromatogram of 1:1 M sodium caseinate:5-CGA sample ( $\mathrm{pH} 6.7,72{ }^{\circ} \mathrm{C}$ ). Unknown peak detected at $R T \sim 9.5 \mathrm{~min}$. Undetected peaks are labeled in parentheses.

In green coffee extract samples, caffeine was observed at the expected RT, but the sample also contained many other peaks with a comparable peak height to caffeine that were not observed in the blank or sodium caseinate control and were thus not identified (Figure 4.31). These peaks were also not integrated nor used in the data analysis. 3-CGA, 4-CGA and 5-CGA isomers were observed in larger quantities in the green coffee extract samples with sodium caseinate. The peak areas of all CGA isomers were added together to quantify the total CGA concentration in samples. A sample of the pure green coffee extract with no sodium caseinate afforded all observable CGA isomers (Figure 4.32). 


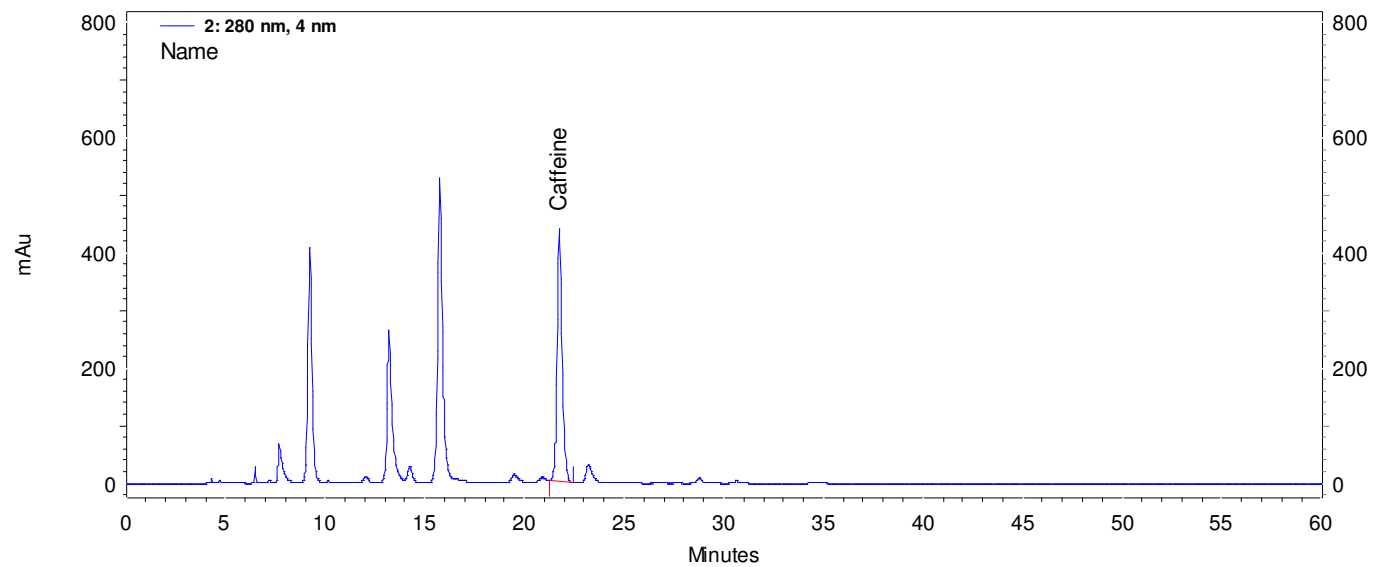

Figure 4.31. Sodium caseinate and green coffee extract sample at $280 \mathrm{~nm}$ (pH 6.7, $72^{\circ} \mathrm{C}$, rep 3).

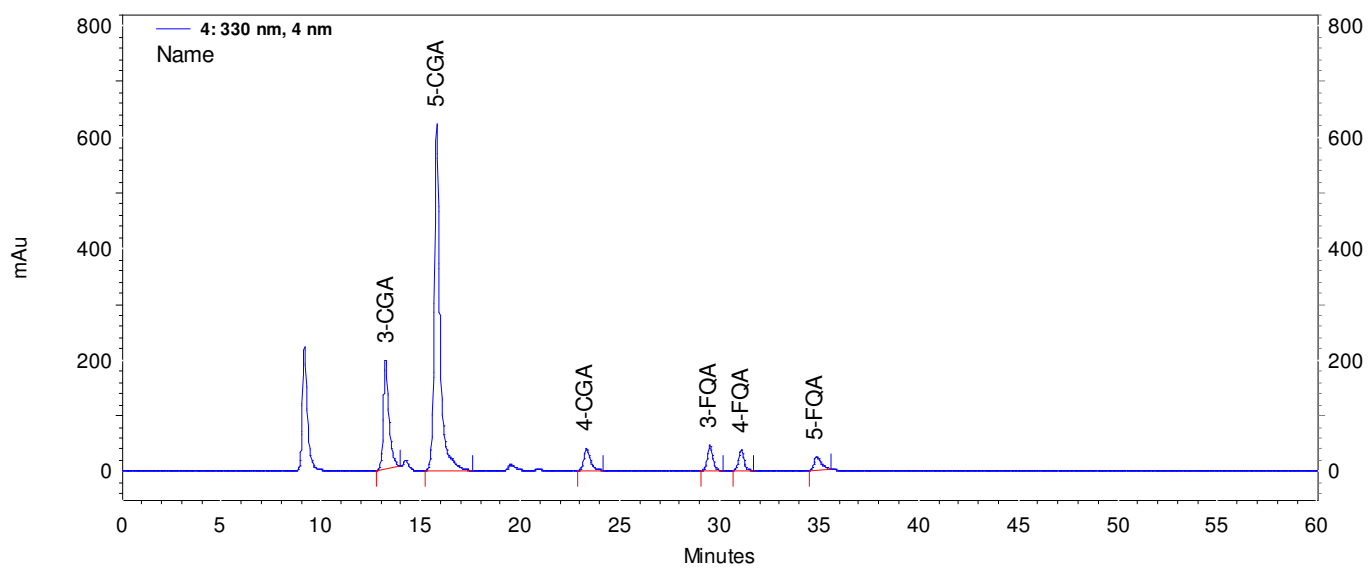

Figure 4.32. Sample chromatogram of green coffee extract only measured at 330 $\mathrm{nm}$.

\subsection{BCA trials}

BCA trials were performed to confirm the absence of interference from soluble proteins remaining in the sample supernatant. The concentration of protein present in each sample was calculated using a standard curve of BSA, constructed prior to sample analysis (Figure 4.33). The absorbance of the blank solution was subtracted from the absorbance of each standard and sample to calculate the final protein concentration. The amount of protein from each sample ranged between $1-6 \%$ of the original sodium caseinate solution and was thus not 
considered to be an interfering substance during caffeine or 5-CGA quantification (Table 4.4 and Table 4.5). In addition, a sodium dodecyl sulfate polyacrylamide gel electrophoresis (SDS-PAGE) analysis was run to qualitatively assess the amount of impurities (i.e. whey proteins) present in the original sodium caseinate powder used. The SDS-PAGE eletrophoretogram depicted in Figure 4.34 shows sodium caseinate with various dilutions that contained impurities present in the powder at relatively low intensities compared to sodium caseinate. This qualitative assessment showed any impurities or soluble proteins that remained in the supernatant after isoelectric precipitation of the caseins did not total to a significant amount and thus would not affect any interpretation of the data from these experiments.

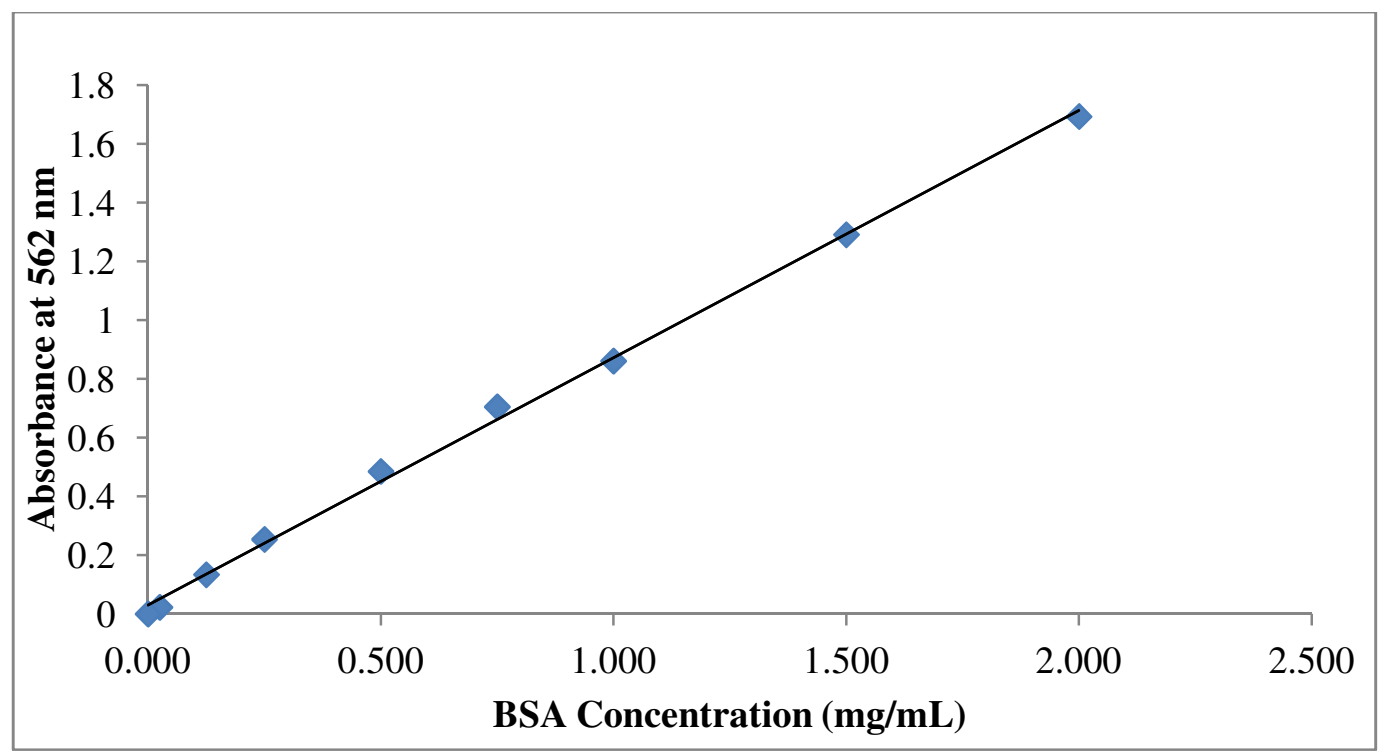

Figure 4.33. BSA standard curve of absorbance at $562 \mathrm{~nm}$ vs. concentration $(\mathrm{mg} / \mathrm{mL}) \cdot R^{2}=0.9980$. 
Table 4.4. BCA protein assay of 1:1 M sodium caseinate:caffeine samples

\begin{tabular}{ccccc}
\hline Repetition & $\mathbf{p H}$ & $\begin{array}{c}\text { Temperature } \\
\left({ }^{\circ} \mathbf{C}\right)\end{array}$ & $\begin{array}{c}\text { Concentration } \\
(\mathbf{m g} / \mathbf{m L})\end{array}$ & $\begin{array}{c}\text { Protein } \\
(\mathbf{\%})\end{array}$ \\
\hline 1 & 6.7 & 25 & 0.2756 & $1.01 \pm 0.00$ \\
2 & 6.7 & 25 & 0.3018 & $1.11 \pm 0.00$ \\
1 & 6.7 & 72 & 0.3790 & $1.39 \pm 0.00$ \\
2 & 6.7 & 72 & 0.3671 & $1.35 \pm 0.00$ \\
1 & 4.4 & 25 & 0.2566 & $0.94 \pm 0.00$ \\
2 & 4.4 & 25 & 0.3077 & $1.13 \pm 0.00$ \\
1 & 4.4 & 72 & 0.3006 & $1.10 \pm 0.00$ \\
2 & 4.4 & 72 & 0.3434 & $1.26 \pm 0.00$ \\
\hline
\end{tabular}

Table 4.5. BCA protein assay of 1:1 M sodium caseinate:5-CGA samples

\begin{tabular}{ccccc}
\hline Repetition & $\mathbf{p H}$ & $\begin{array}{r}\text { Temperature } \\
\left({ }^{\circ} \mathbf{C}\right)\end{array}$ & $\begin{array}{c}\text { Concentration } \\
(\mathbf{m g} / \mathbf{m L})\end{array}$ & $\begin{array}{c}\text { Protein } \\
(\%)\end{array}$ \\
\hline 1 & 6.7 & 25 & 1.3120 & $4.82 \pm 0.00$ \\
2 & 6.7 & 25 & 1.2894 & $4.74 \pm 0.00$ \\
1 & 6.7 & 72 & 1.5319 & $5.63 \pm 0.00$ \\
2 & 6.7 & 72 & 1.5461 & $5.68 \pm 0.00$ \\
1 & 4.4 & 25 & 1.2133 & $4.46 \pm 0.00$ \\
2 & 4.4 & 25 & 1.2407 & $4.56 \pm 0.00$ \\
1 & 4.4 & 72 & 1.4035 & $5.16 \pm 0.00$ \\
2 & 4.4 & 72 & 1.4510 & $5.33 \pm 0.00$ \\
\hline
\end{tabular}




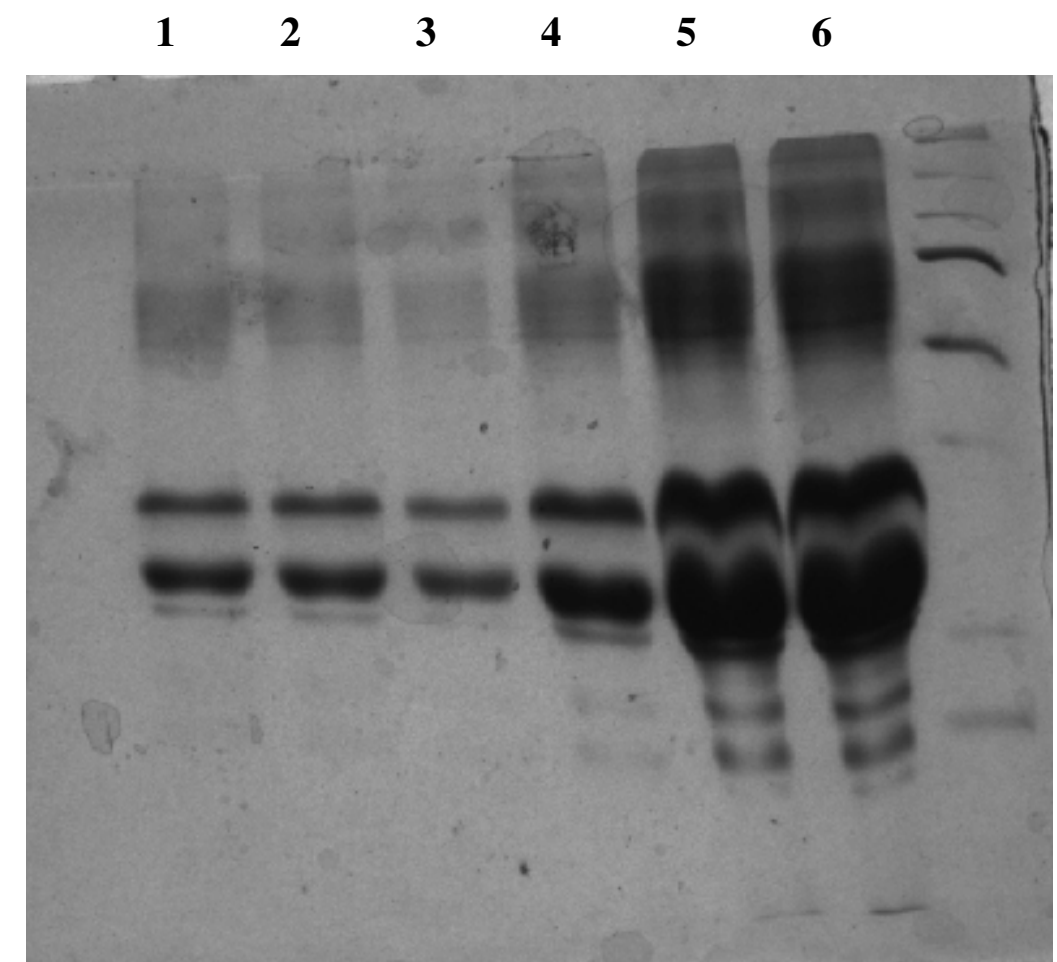

Figure 4.34. SDS-PAGE eletrophoretogram of sodium caseinate with various dilutions used in all experiments. Lane $1=$ most dilute sample of sodium caseinate; lane $6=$ least dilute sample of sodium caseinate. Impurities become less evident in diluted samples. The level of intensity of impurities in all lanes is markedly less than that of sodium caseinate. The far right lane depicts the molecular marker (ladder). 


\subsection{CONCLUSIONS}

Rates of coffee consumption worldwide continue to increase due to the uniquely satisfying flavor of coffee. Though most coffee consumers may not be aware of it, the bioactive compounds in coffee actually serve many important physiological functions in maintaining overall health. These benefits include hepatoprotective, hypoglycemic, and antiviral activity. Clearly, these health benefits warrant further investigation into exploiting their beneficial activities and imparting them from foods other than from coffee. Milk proteins have been remarkable at adapting to their evolutionary task of binding and transporting vital micronutrients from the mother to the neonate, thus the binding capacity of casein to caffeine and CGA and its use as a bioactive transport vehicle was the primary objective of this thesis. However, a procedure was first established to bind caffeine and CGA to sodium caseinate by determining the optimal chemical environment ( $\mathrm{pH}$ and temperature) that would result in increased binding interactions based on the investigated treatment conditions. The steps in the developed procedure are both simple to carry out and relatively efficient thus providing a protocol that is neither time consuming nor labor intensive. The main effect of temperature was shown to have a significant effect on the concentration of caffeine detected in the supernatant and therefore the concentration that remained bound to sodium caseinate $(\mathrm{p}<0.01)$. This suggests that binding of caffeine to sodium caseinate is likely via hydrophobic interactions. Conversely, the interaction effect of $\mathrm{pH}$ and temperature was shown to have a significant effect on the concentration of CGA detected in the supernatant and therefore the 
concentration that remained bound to sodium caseinate $(\mathrm{p}<0.01)$. This suggests the binding mechanism for CGA is likely a result of both electrostatic and hydrophobic interactions with sodium caseinate. The main effect of $\mathrm{pH}$ alone was not found to be statistically significant $(\mathrm{p}>0.01)$ for either caffeine or CGA, indicating that electrostatic interactions alone are not likely to contribute to the binding mechanism. However, the main effect of $\mathrm{pH}$ may not have been statistically significant due to the fact that all samples were standardized to a final $\mathrm{pH}$ at 4.6 to precipitate the caseins. This may affect any reversible binding events that originally occurred at the initial investigated levels of $\mathrm{pH}$. Essentially, the binding procedures provided herein may not have sufficiently shown the true effects of $\mathrm{pH}$ due to the inability to measure binding that occurred at the investigated levels of $\mathrm{pH}$ but rather, these procedures allowed only for binding measurements after isoelectric precipitation of the caseins, and therefore an estimated amount of binding that occurred at the final $\mathrm{pH}$ of 4.6.

The difference in binding mechanisms between caffeine and 5-CGA to sodium caseinate could be attributed to the fundamental differences in their chemical structure. Compared to caffeine, 5-CGA contains a carboxylic acid $(-\mathrm{COOH})$ functional group which can remain protonated or easily deprotonated by being in a chemical environment with a $\mathrm{pH}$ greater than its $\mathrm{pKa}(\sim 3.5)$. Because the pKa of 5-CGA is relatively low, changes in its ionic state can be easily influenced by a smaller change in the $\mathrm{pH}$ of its chemical environment and thus influence binding with other molecules by electrostatic interactions. Conversely, the $\mathrm{pKa}$ of protonated amines are $\sim 14$ thus much larger changes to 
the $\mathrm{pH}$ of its chemical environment are needed to influence electrostatic interactions. The small difference in electronegativity between the bonded atoms in the caffeine molecule makes it a relatively nonpolar compound. Thus it is plausible that caffeine binds to hydrophobic regions of the casein micelle once they have been exposed upon denaturation at higher temperatures. The prevalent binding mechanism between caffeine and the nonpolar regions of sodium caseinate are thus likely via hydrophobic interactions.

Application of the optimal experimental conditions on a sample of green coffee extract which contained naturally occurring caffeine and CGA was performed to verify comparable binding capacity and reproducibility of the method as sodium caseinate with commercial standards of the bioactive compounds. Results from these experiments were in good agreement with the results from binding commercial standards of caffeine and 5-CGA to sodium caseinate. Simultaneous determination of caffeine and 5-CGA was achieved through the development, optimization, and validation of an analytical procedure by RP-HPLC. This method used common reagents that are readily accessible by most laboratories and provided a sensitive method for accurately quantifying both caffeine and CGA through demonstrating good results from validation studies that included: linearity, intra-assay precision, and selectivity.

Results from these experiments provide but a modest contribution to the immense potential applications of coffee bioactive compounds in dairy products. Indeed, the procedures provided herein for binding caffeine and 5-CGA to sodium caseinate serves as a preliminary starting point for many more research endeavors 
involving these health-promoting and versatile compounds. The continual development of sensitive analytical techniques for accurate quantification of molecules is needed by researchers across all disciplines of science, both in academia and industry. Thus through active collaboration of scientists from interdisciplinary backgrounds, such as those involved in this thesis work, insights into additional ways of incorporating the ever versatile casein micelle with other health promoting molecules is promising. 


\subsection{DIRECTIONS FOR FUTURE RESEARCH}

- $\quad$ Explore additional levels of the main effects of $\mathrm{pH}$ and temperature to observe for possible trending in the extent of binding between caffeine and 5-CGA with sodium caseinate

- Hold insignificant factors constant while varying significant factors at larger ranges and employing a response surface model (RSM) to determine optimum treatment levels

- Vary the molar ratio of coffee bioactive to sodium caseinate beyond a 1:1 $M$ ratio to observe any differences in the effects of using different concentrations of each compound

- Investigate whether a specific fraction of casein is responsible for binding caffeine and 5-CGA by using commercial standards of $\alpha$ - and $\beta$-caseins

- Direct measurement of the casein pellet for the amount of bound bioactive compound

- Utilize additional characterization techniques such as dynamic light scattering (DLS) particle size analysis on changes to the casein micelle size and structure

- $\quad$ Prepare heated samples using a stirring water bath to maintain better temperature control compared to using a hot plate

- Study whether the same binding interactions occur with other bioactive compounds in coffee, such as caffeic acid 


\subsection{REFERENCES}

Almeida, A. A. P., Farah, A., Silva, D. A. M., Nunan, E. A., \& Gloria, M. B. A. (2006). Antibacterial activity of coffee extracts and selected coffee chemical compounds against enterobacteria. Journal of Agricultural and Food Chemistry, 54(23), 8738-8743. Retrieved Nov, from Article database.

Alonso-Salces, R. M., Guillou, C., \& Berrueta, L. A. (2009). Liquid chromatography coupled with ultraviolet absorbance detection, electrospray ionization, collision-induced dissociation and tandem mass spectrometry on a triple quadrupole for the on-line characterization of polyphenols and methylxanthines in green coffee beans. Rapid Communications in Mass Spectrometry, 23(3), 363-383. Retrieved Feb, from Article database.

[AOAC] Association of official analytical chemists. 2000. Official Methods of Analysis. $17^{\text {th }}$ ed., AOAC International, Gaithersburg, MD.

Belitz, H. D., Grosch, W., \& Schieberle, P. (2009). Food chemistry. 4th revised and extended edition. Food chemistry. 4th revised and extended edition., Ed. 4, xliv + 1070pp. Book database.

Benshitrit, R. C., Levi, C. S., Tal, S. L., Shimoni, E., \& Lesmes, U. (2012). Development of oral food-grade delivery systems: Current knowledge and future challenges. Food \& Function, 3(1), 10-21. Retrieved Jan, from Review database.

Berbert, P. A., Queiroz, D. M., Sousa, E. F., Molina, M. B., Melo, E. C., \& Faroni, L. R. D. (2001). Dielectric properties of parchment coffee. Journal of Agricultural Engineering Research, 80(1), 65-80. Retrieved Sep, from Article database.

Brew, K., (2003). $\alpha$-Lactalbumin. In Advanced Dairy Chemistry, Volume 1, Proteins. Third Edition, New York, Kluwer Academic/Plenum Publishers, pp 387-412.

Cardenas, C., Quesada, A. R., \& Medina, M. A. (2011). Anti-angiogenic and antiinflammatory properties of Kahweol, a coffee diterpene. Plos One, 6(8), 9. Retrieved Aug, from Article database.

Cheng, B. A., Liu, X. R., Gong, H., Huang, L. Q., Chen, H., Zhang, X., Chuanzhou, L., Yang, M., Ma, B., Jiao, L., Zheng, L., Huang, K. (2011). Coffee components inhibit Amyloid formation of human Islet Amyloid polypeptide in vitro: Possible link between coffee consumption and 
Diabetes Mellitus. Journal of Agricultural and Food Chemistry, 59(24), 13147-13155. Retrieved Dec, from Article database.

Clark, A., Lewis, C. E., Willis, A. C., Cooper, G. J. S., Morris, J. F., Reid, K. B. M., et al. (1987). Islet Amyloid formed from diabetes-associated peptide may be pathogenic in Type-2 Diabetes. Lancet, 2(8553), 231-234.

Retrieved Aug, from Article database.

Clifford, M. N. (1985). Chemical and physical aspects of green coffee and coffee products. Clifford, M. N. and K. C. Willson (Ed.). Coffee: Botany, Biochemistry and Production of Beans and Beverage. $X v+457 p$. the Avi Publishing Company, Inc.: Westport, Conn., USA; Croom Helm Ltd.: Beckenham, Kent, England; Croom Helm Australia Pty Ltd.: Sydney, N.S.W., Australia. Illus. Maps, 305-374. Book database.

Dacarro, C., Daglia, M., Cuzzoni, M. T., \& Bonferoni, B. (1995). Antibacterial activity of coffee against Streptococcus mutans. Igiene Moderna, 104(3), 379-387. Article database.

Daglia, M., Papetti, A., Grisoli, P., Aceti, C., Spini, V., Dacarro, C., et al. (2007). Isolation, identification, and quantification of roasted coffee antibacterial compounds. Journal of Agricultural and Food Chemistry, 55(25), 1020810213. Retrieved Dec, from Article database.

Danhelova, H., Hradecky, J., Prinosilova, S., Cajka, T., Riddellova, K., Vaclavik, L., et al. (2012). Rapid analysis of caffeine in various coffee samples employing direct analysis in real-time ionization-high-resolution mass spectrometry. Analytical and Bioanalytical Chemistry, 403(10), 28832889. Retrieved Jul, from Article database.

Dews, P. B. (1984). Caffeine, Perspectives from Recent Research. SpringerVerlag, Berlin Heidelberg.

Duarte, G. S., Pereira, A. A., \& Farah, A. (2010). Chlorogenic acids and other relevant compounds in Brazilian coffees processed by semi-dry and wet post-harvesting methods. Food Chemistry, 118(3), 851-855. Retrieved Feb, from Article database.

Esquivel, P., \& Jimenez, V. M. (2012). Functional properties of coffee and coffee by-products. Food Research International, 46(2), 488-495. Review database.

Farah, A., \& Donangelo, C. M. (2006). Phenolic compounds in coffee. Brazilian Journal of Plant Physiology, 18(1), 23-36. Retrieved Jan-Mar, from Article database. 
Farah, A., De Paulis, T., Trugo, L. C., \& Martin, P. R. (2005). Effect of roasting on the formation of chlorogenic acid lactones in coffee. Journal of Agricultural and Food Chemistry, 53(5), 1505-1513. Retrieved Mar, from Article database.

Fox, P. F. (2003). Milk proteins: General and historical aspects. In Advanced Dairy Chemistry, Volume 1 - Proteins, Third Edition, New York, Kluwer Academic/Plenum Publishers, pp. 1-41.

Fox, P. F., \& McSweeney, P. L. H., (1998). Dairy Chemistry and Biochemistry. London, Blackie Academic \& Professional.

Frost-Meyer, N. J., \& Logomarsino, J. V. (2012). Impact of coffee components on inflammatory markers: A review. Journal of Functional Foods, 4(4), 819830. Review database.

Gielissen, R., \& Graafland, J. (2009). Concepts of price fairness: Empirical research into the Dutch coffee market. Business Ethics-a European Review, 18(2), 165-178. Retrieved Apr, from Article database.

Govindarajan, V. S. (1980). Turmeric-Chemistry, technology, and quality. CRC Critical Reviews in Food Science and Nutrition, 12(3), 199-301.

Greenberg, J. A., Axen, K. V., Schnoll, R., \& Boozer, C. N. (2005). Coffee, tea and diabetes: The role of weight loss and caffeine. International Journal of Obesity, 29(9), 1121-1129. Retrieved Sep, from Article database.

Hernan, M. A., Takkouche, B., Caamano-Isorna, F., \& Gestal-Otero, J. J. (2002). A meta-analysis of coffee drinking, cigarette smoking, and the risk of Parkinson's disease. Annals of Neurology, 52(3), 276-284. Retrieved Sep, from Article database.

Higdon, J. V., \& Frei, B. (2006). Coffee and health: A review of recent human research. Critical Reviews in Food Science and Nutrition, 46(2), 101-123. Review database.

Holt, C. (1994). The biological function of casein? Yearbook 1994., 60-68. Annual report database.

Holt, C., \& Horne, D. S. (1996). The hairy casein micelle: Evolution of the concept and its implications for dairy technology. Netherlands Milk and Dairy Journal, 50(2), 85-111. Review database. 
Hurley, W. L., (2003). Immunoglobulins in mammary secretions. In Advanced Dairy Chemistry, Volume 1, Proteins. Third Edition, New York, Kluwer Academic/Plenum Publishers, pp 422-439

Istarova, T. A., Semenova, M. G., Sorokoumova, G. M., Selishcheva, A. A., Belyakova, L. E., Polikarpov, Y. N., et al. (2005). Effect of pH on the interactions of sodium caseinate with soy phospholipids in relation to the foaming ability of their mixtures. Food Hydrocolloids, 19(3), 429-440.

James, J. E. (2004). Critical review of dietary caffeine and blood pressure: A relationship that should be taken more seriously. Psychosomatic Medicine, 66(1), 63-71. Retrieved Jan-Feb, from Review database.

Jaquet, M., Rochat, I., Moulin, J., Cavin, C., \& Bibiloni, R. (2009). Impact of coffee consumption on the gut microbiota: A human volunteer study. International Journal of Food Microbiology, 130(2), 117-121. Retrieved Mar, from Article database.

Lai, Y. S., Hsu, W. H., Huang, J. J., \& Wu, S. C. (2012). Antioxidant and antiinflammatory effects of pigeon pea (Cajanus cajan $L$.) extracts on hydrogen peroxide- and lipopolysaccharide-treated RAW264.7 macrophages. Food \& Function, 3(12), 1294-1301. Retrieved Dec, from Article database.

Lean, M. E. J., \& Crozier, A. (2012). Coffee, caffeine and health: What's in your cup? Maturitas, 72(3), 171-172. Retrieved Jul, from Editorial Material database.

Lee, K. J., \& Jeong, H. G. (2007). Protective effects of kahweol and cafestol against hydrogen peroxide-induced oxidative stress and DNA damage. Toxicology Letters, 173(2), 80-87. Retrieved Sep, from Article database.

Leloup, V., Louvrier, A., \& Liardon, R. (1995). Degradation mechanisms of chlorogenic acids during roasting. In 16th International Scientific Colloquium on Coffee Vols. 1 and 2 (pp. 192-198): Association Scientifique Internationale du Cafe (ASIC) $\{a\}, 42$, rue Scheffer, Paris, France.

Livney, Y. D. (2010). Milk proteins as vehicles for bioactives. Current Opinion in Colloid and Interface Science, 15(1-2), 73-83. Review database.

Lucas, M., Mirzaei, F., Pan, A., Okereke, O. I., Willett, W. C., O'Reilly, E. J., et al. (2011). Coffee, caffeine, and risk of depression among women. Archives of Internal Medicine, 171(17), 1571-1578. Retrieved Sep, from Article database. 
L $\square$ nnerdal, B. (2003). Lactoferrin. In Advanced Dairy Chemistry, Volume 1, Proteins. Third Edition, New York, Kluwer Academic/Plenum Publishers, pp. 449-461.

Maheshwari, R. K., Singh, A. K., Gaddipati, J., \& Srimal, R. C. (2006). Multiple biological activities of curcumin: A short review. Life sciences, 78(18), 2081-2087.

Moon, J. K., Yoo, H. S., \& Shibamoto, T. (2009). Role of roasting conditions in the level of chlorogenic acid content in coffee beans: Correlation with coffee acidity. Journal of Agricultural and Food Chemistry, 57(12), 53655369. Retrieved Jun, from Article database.

O'Connell, J. E., Grinberg, V. Y., \& de Kruif, C. G. (2003). Association behavior of beta-casein. Journal of Colloid and Interface Science, 258(1), 33-39. Retrieved Feb, from Article database.

Pollock, B. G., Wylie, M., Stack, J. A., Sorisio, D. A., Thompson, D. S., Kirshner, M. A., et al. (1999). Inhibition of caffeine metabolism by estrogen replacement therapy in postmenopausal women. Journal of Clinical Pharmacology, 39(9), 936-940. Retrieved Sep, from Article database.

Ramalakshmi, K., Hithamani, G., Asha, K. R., \& Rao, L. J. M. (2011). Separation and characterisation of chlorogenic acid-rich conserves from green coffee beans and their radical scavenging potential. International Journal of Food Science and Technology, 46(1), 109-115. Retrieved Jan, from Article database.

Rosengren, A., Dotevall, A., Wilhelmsen, L., Thelle, D., \& Johansson, S. (2004). Coffee and incidence of diabetes in Swedish women: A prospective 18year follow-up study. Journal of Internal Medicine, 255(1), 89-95. Retrieved Jan, from Article database.

Rowland, S. J. (1938). The determination of the nitrogen distribution in milk. Journal of Dairy Research, 9(42-6).

Salazar-Martinez, E., Willett, W. C., Ascherio, A., Manson, J. E., Leitzmann, M. F., Stampfer, M. J., et al. (2004). Coffee consumption and risk for type 2 diabetes mellitus. Annals of Internal Medicine, 140(1), 1-8. Retrieved Jan, from Article database.

Samanidou, V., Tsagiannidis, A., \& Sarakatsianos, I. (2012). Simultaneous determination of polyphenols and major purine alkaloids in Greek Sideritis species, herbal extracts, green tea, black tea, and coffee by high- 
performance liquid chromatography-diode array detection. Journal of Separation Science, 35(4), 608-615. Retrieved Feb, from Article database.

Semo, E., Kesselman, E., Danino, D., \& Livney, Y. D. (2007). Casein micelle as a natural nano-capsular vehicle for nutraceuticals. Food Hydrocolloids, 21(5-6), 936-942. Retrieved Jul-Aug, from Article; Proceedings Paper database.

Smith, P. K., Krohn, R. I., Hermanson, G. T., Mallia, A. K., Gartner, F. H., Provenzano, M. D., et al. (1985). Measurement of protein using bicinchoninic acid. Analytical Biochemistry, 150(1), 76-85. Article database.

Southward, C. R. (1985). Manufacture and application of edible casein products .1. Manufacture and properties. New Zealand Journal of Dairy Science and Technology, 20(2), 79-101. Review database.

Sugiarto, M., Ye, A., \& Singh, H. (2009). Characterisation of binding of iron to sodium caseinate and whey protein isolate. Food Chemistry, 114(3), 10071013.

Swadesh, J. K., Stewart, Jr., C. W., \& Uden, P. C. (1993). Comparison of liquid chromatographic methods for analysis of homologous $n$-alkyl esters of biphenyl-4,4'-dicarboxylic acid. Analyst, 118, 1123.

Swaisgood, H. E. (2003). Chemistry of the caseins. In Advanced Dairy Chemistry, Volume 1, Proteins. Third Edition, New York, Kluwer Academic/Plenum Publishers, pp. 139-187.

Trejo, R., Dokland, T., Jurat-Fuentes, J., \& Harte, F. (2011). Cryo-transmission electron tomography of native casein micelles from bovine milk. Journal of Dairy Science, 94(12), 5770-5775. Retrieved Dec, from Article database.

Trugo, L. C., \& Macrae, R. (1984). A study of the effect of roasting on the chlorogenic acid composition of coffee using HPLC. Food Chemistry, 15(3), 219-227. Article database.

Trugo, L. C., Maria, C. A. B. d., \& Werneck, C. C. (1991). Simultaneous determination of total chlorogenic acid and caffeine in coffee by high performance gel filtration chromatography. Food Chemistry, 42(1), 81-87. 
Vaast, P., Bertrand, B., Perriot, J. J., Guyot, B., \& Genard, M. (2006). Fruit thinning and shade improve bean characteristics and beverage quality of coffee (Coffea arabica L.) under optimal conditions. Journal of the Science of Food and Agriculture, 86(2), 197-204. Retrieved Jan, from Article database.

World Resources Institute. (2011). Earth trends data tables: Energy and resources. Retrieved Mar. from Article database.

Yazdi, S. R., \& Corredig, M. (2012). Heating of milk alters the binding of curcumin to casein micelles. A fluorescence spectroscopy study. Food Chemistry, 132(3), 1143-1149. Retrieved Jun, from Article database.

Ye, A., Edwards, P. J. B., Gilliland, J., Jameson, G. B., \& Singh, H. (2012). Temperature-dependent complexation between sodium caseinate and gum arabic. Food Hydrocolloids, 26(1), 82-88.

Zimet, P., Rosenberg, D., \& Livney, Y. D. (2011). Re-assembled casein micelles and casein nanoparticles as nano-vehicles for omega-3 polyunsaturated fatty acids. Food Hydrocolloids, 25(5), 1270-1276. Retrieved Jul, from Article database. 
Appendix A: Data for precision studies on caffeine and 5-CGA

\begin{tabular}{|c|c|c|c|c|c|c|}
\hline Sample Name & $\begin{array}{l}\text { Known Conc. } \\
(\mathrm{mg} / \mathrm{mL})\end{array}$ & $\begin{array}{l}\text { Observed Conc. } \\
\text { (mg/mL) }\end{array}$ & Peak Area & $\begin{array}{l}\text { Average } \\
\text { Peak Area }\end{array}$ & $\begin{array}{l}\text { Relative Standard } \\
\text { Deviation (\% RSD) }\end{array}$ & $\begin{array}{c}\text { Precision } \\
\text { Range }\end{array}$ \\
\hline Caff A1 & 0.0625 & 0.071913 & $11,871,663$ & \multirow{5}{*}{$11,875,780$} & \multirow{5}{*}{0.05} & \multirow{5}{*}{$<1 \%$} \\
\hline Caff A2 & 0.0625 & 0.071901 & $11,869,737$ & & & \\
\hline Caff A3 & 0.0625 & 0.071968 & $11,879,946$ & & & \\
\hline Caff A4 & 0.0625 & 0.071936 & $11,875,076$ & & & \\
\hline Caff A5 & 0.0625 & 0.071985 & $11,882,476$ & & & \\
\hline Caff B1 & 0.03125 & 0.033021 & $6,002,144$ & \multirow{5}{*}{$6,000,843$} & \multirow{5}{*}{0.04} & \multirow{5}{*}{$<1 \%$} \\
\hline Caff B2 & 0.03125 & 0.033018 & $6,001,681$ & & & \\
\hline Caff B3 & 0.03125 & 0.033010 & $6,000,485$ & & & \\
\hline Caff B4 & 0.03125 & 0.032986 & $5,996,814$ & & & \\
\hline Caff B5 & 0.03125 & 0.033027 & $6,003,092$ & & & \\
\hline Caff C1 & 0.015625 & 0.013204 & $3,011,482$ & \multirow{5}{*}{$3,012,412$} & \multirow{5}{*}{0.05} & \multirow{5}{*}{$<1 \%$} \\
\hline Caff C2 & 0.015625 & 0.013207 & $3,011,870$ & & & \\
\hline Caff C3 & 0.015625 & 0.013206 & $3,011,713$ & & & \\
\hline Caff C4 & 0.015625 & 0.013229 & $3,015,277$ & & & \\
\hline Caff C5 & 0.015625 & 0.013206 & $3,011,719$ & & & \\
\hline
\end{tabular}

\begin{tabular}{|c|c|c|c|c|c|c|}
\hline Sample Name & $\begin{array}{l}\text { Known Conc. } \\
(\mathrm{mg} / \mathrm{mL})\end{array}$ & $\begin{array}{l}\text { Observed Conc. } \\
(\mathrm{mg} / \mathrm{mL})\end{array}$ & Peak Area & $\begin{array}{l}\text { Average } \\
\text { Peak Area }\end{array}$ & $\begin{array}{l}\text { Relative Standard } \\
\text { Deviation (\% RSD) }\end{array}$ & $\begin{array}{c}\text { Precision } \\
\text { Range }\end{array}$ \\
\hline CGA A1 & 0.0625 & 0.065321 & $14,860,698$ & \multirow{5}{*}{$14,829,745$} & \multirow{5}{*}{0.75} & \multirow{5}{*}{$<1 \%$} \\
\hline CGA A2 & 0.0625 & 0.065782 & $14,978,295$ & & & \\
\hline CGA A3 & 0.0625 & 0.065130 & $14,811,928$ & & & \\
\hline CGA A4 & 0.0625 & 0.065207 & $14,831,589$ & & & \\
\hline CGA A5 & 0.0625 & 0.064558 & $14,666,214$ & & & \\
\hline CGA B1 & 0.03125 & 0.034415 & $6,978,188$ & \multirow{5}{*}{$7,007,503$} & \multirow{5}{*}{0.74} & \multirow{5}{*}{$<1 \%$} \\
\hline CGA B2 & 0.03125 & 0.034718 & $7,055,321$ & & & \\
\hline CGA B3 & 0.03125 & 0.034776 & $7,070,062$ & & & \\
\hline CGA B4 & 0.03125 & 0.034312 & $6,951,698$ & & & \\
\hline CGA B5 & 0.03125 & 0.034431 & $6,982,247$ & & & \\
\hline CGA C1 & 0.015625 & 0.019591 & $3,197,140$ & \multirow{5}{*}{$3,210,977$} & \multirow{5}{*}{1.33} & \multirow{5}{*}{$1.33 \%$} \\
\hline CGA C2 & 0.015625 & 0.019706 & $3,226,576$ & & & \\
\hline CGA C3 & 0.015625 & 0.019664 & $3,215,890$ & & & \\
\hline CGA C4 & 0.015625 & 0.019403 & $3,149,330$ & & & \\
\hline CGA C5 & 0.015625 & 0.019861 & $3,265,950$ & & & \\
\hline
\end{tabular}


Appendix B: Statistical analysis by JMP - binding caffeine to sodium caseinate trials

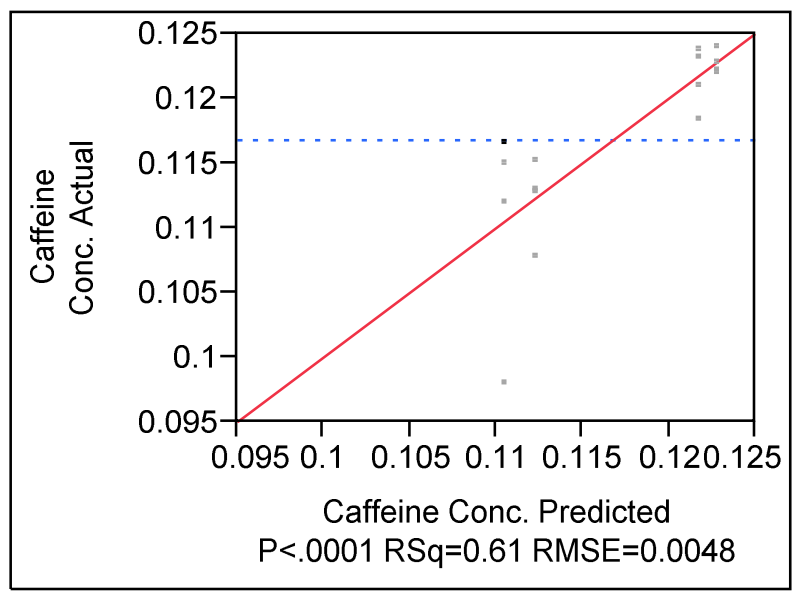

\section{Summary of Fit}

RSquare

0.611634

RSquare Adj

0.514543

Root Mean Square Error

Mean of Response

0.00478

0.116781

Observations (or Sum Wgts)

16

Parameter Estimates

Term

Intercept

$\mathrm{pH}[4.4]$

Temperature[25]

$\mathrm{pH}[4.4] *$ Temperature[25]

$\begin{array}{rr}\text { Estimate } & \text { Std Error } \\ 0.1167813 & 0.000906 \\ -0.000156 & 0.001195 \\ 0.0054562 & 0.001195 \\ 0.0007187 & 0.001195\end{array}$

DFDen

\section{1}

t Ratio

128.86

Prob $>|t|$

$11-0.13 \quad 0.8983$

$11 \quad 4.57 \quad 0.0008 *$

$\begin{array}{lll}11 & 0.60 & 0.5597\end{array}$

\section{Fixed Effect Tests}

Source

$\mathrm{pH}$

Temperature

pH*Temperature

$\begin{array}{rrrrr}\text { Nparm } & \text { DF } & \text { DFDen } & \text { F Ratio } & \text { Prob > F } \\ 1 & 1 & 11 & 0.0171 & 0.8983 \\ 1 & 1 & 11 & 20.8471 & 0.0008^{*} \\ 1 & 1 & 11 & 0.3618 & 0.5597\end{array}$


pH

Leverage Plot

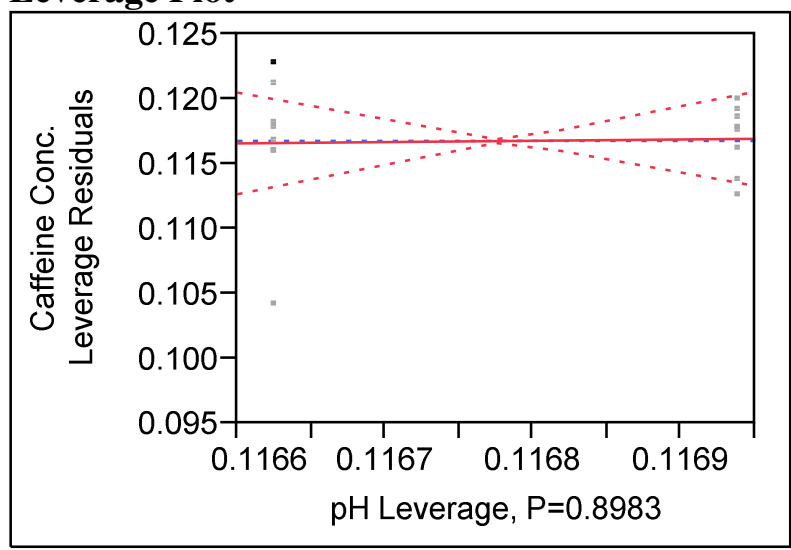

Least Squares Means Table

Level Least Sq Mean

Std Error

$4.4 \quad 0.11662500$

0.00149978

6.7

0.11693750

0.00149978

\section{LS Means Plot}

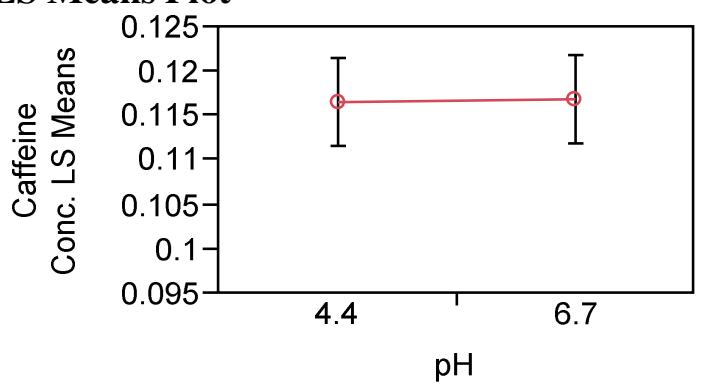

Page 104 


\section{LSMeans Differences Student's t}

$\alpha=0.017$

LSMean[i] By LSMean[i]

\begin{tabular}{|l|r|r|}
\hline $\begin{array}{l}\text { Mean[i]-Mean[j] } \\
\text { Std Err Dif } \\
\text { Lower CL Dif } \\
\text { Upper CL Dif }\end{array}$ & 4.4 & 6.7 \\
\hline 4.4 & 0 & \\
& 0 & -0.0003 \\
& 0 & 0.00239 \\
& 0 & -0.007 \\
\hline 6.7 & 0.00642 \\
& 0.0031 & 0 \\
& -0.0064 & 0 \\
& 0.00705 & 0 \\
\hline
\end{tabular}

Level

Least Sq Mean

6.7

0.11693750

4.4

A

0.11662500

Levels not connected by same letter are significantly different.

\section{Temperature}

Leverage Plot

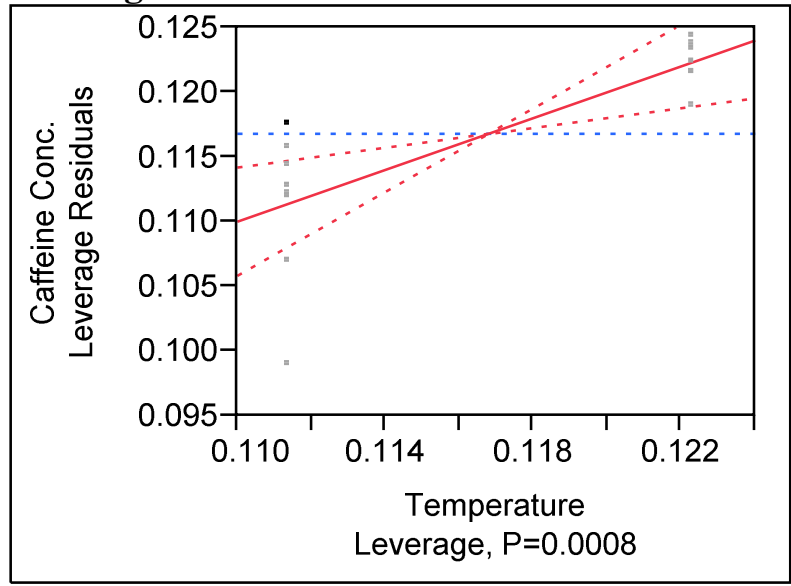

\section{Least Squares Means Table}

Level Least Sq Mean

$25 \quad 0.12223750$

72

0.11132500

Std Error

0.00149978

0.00149978 


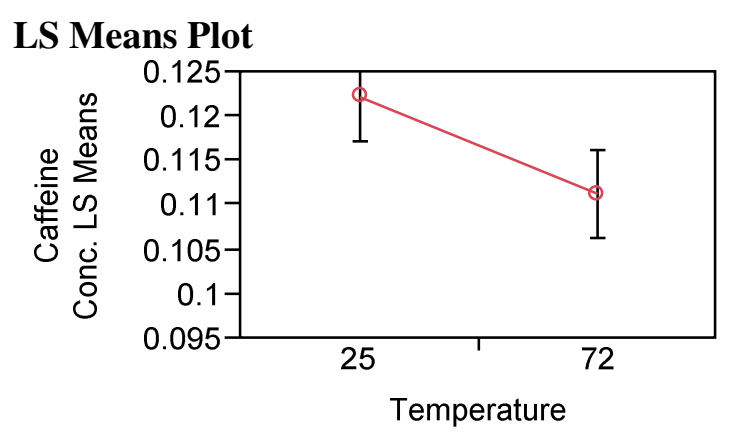

\section{LSMeans Differences Student's t}

$\alpha=0.017$

LSMean[i] By LSMean[j]

\begin{tabular}{|l|r|r|}
\hline Mean[i]-Mean[j] & 25 & 72 \\
Std Err Dif & & \\
Lower CL Dif & & \\
Upper CL Dif & 0 & 0.01091 \\
\hline 25 & 0 & 0.00239 \\
& 0 & 0.00418 \\
& 0 & 0.01765 \\
\hline 72 & -0.0109 & 0 \\
& 0.00239 & 0 \\
& -0.0176 & 0 \\
& -0.0042 & 0 \\
\hline
\end{tabular}

Level

25

72

Levels not connected by same letter are significantly different.

pH*Temperature

Leverage Plot

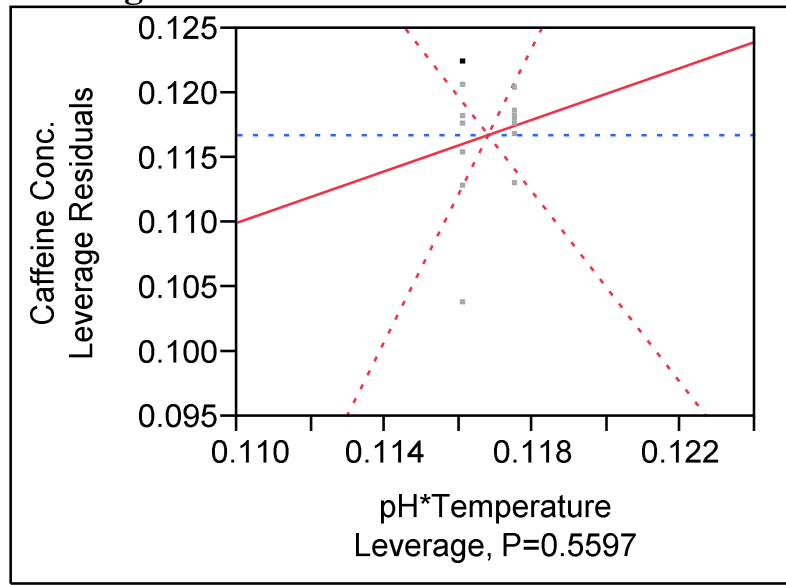

Least Squares Means Table

Level

$4.4,25$

$4.4,72$

$6.7,25$

$6.7,72$

Least Sq Mean

0.12280000

0.11045000

0.12167500

0.11220000
Least Sq Mean

0.12223750

0.11132500 
LS Means Plot

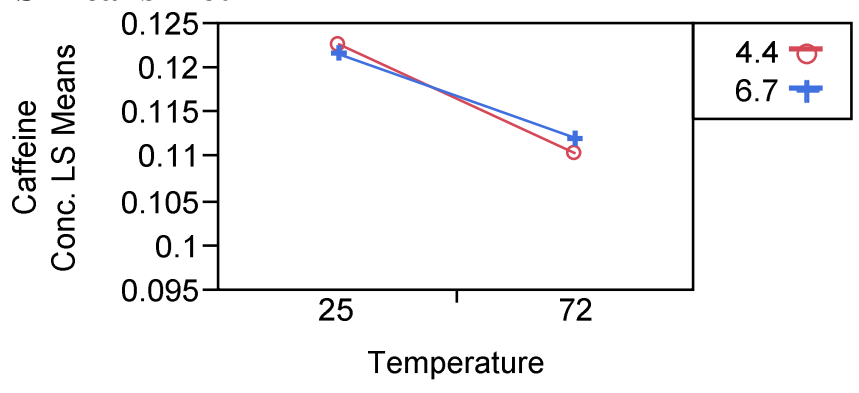

pH*Temperature

LSMeans Differences Tukey HSD

$\alpha=0.017$

LSMean[i] By LSMean[j]

\begin{tabular}{|l|r|r|r|r|}
\hline $\begin{array}{l}\text { Mean[i]-Mean[j] } \\
\text { Std Err Dif } \\
\text { Lower CL Dif } \\
\text { Upper CL Dif }\end{array}$ & $4.4,25$ & $4.4,72$ & $6.7,25$ & $6.7,72$ \\
\hline $4.4,25$ & & & & \\
& 0 & 0.01235 & 0.00112 & 0.0106 \\
& 0 & 0.00338 & 0.00338 & 0.00338 \\
& 0 & $-4 \mathrm{e}-5$ & -0.0113 & -0.0018 \\
& 0 & 0.02474 & 0.01351 & 0.02299 \\
\hline $4.4,72$ & -0.0123 & 0 & -0.0112 & -0.0018 \\
& 0.00338 & 0 & 0.00338 & 0.00338 \\
& -0.0247 & 0 & -0.0236 & -0.0141 \\
& 0.00004 & 0 & 0.00116 & 0.01064 \\
\hline $6.7,25$ & -0.0011 & 0.01123 & 0 & 0.00947 \\
& 0.00338 & 0.00338 & 0 & 0.00338 \\
& -0.0135 & -0.0012 & 0 & -0.0029 \\
& 0.01126 & 0.02361 & 0 & 0.02186 \\
\hline $6.7,72$ & -0.0106 & 0.00175 & -0.0095 & 0 \\
& 0.00338 & 0.00338 & 0.00338 & 0 \\
& -0.023 & -0.0106 & -0.0219 & 0 \\
& 0.00179 & 0.01414 & 0.00291 & 0 \\
\hline
\end{tabular}

Level

$4.4,25$

A

Least Sq Mean

$6.7,25$

A

0.12280000

$6.7,72$

A

0.12167500

$4.4,72$

0.11220000

0.11045000

Levels not connected by same letter are significantly different.

\section{Day}

Least Squares Means Table

Level Least Sq Mean

$1 \quad 0.11745078$

0.11611172

Std Error

0.00061075

2

0.00061075 


\section{Interaction Profiles}

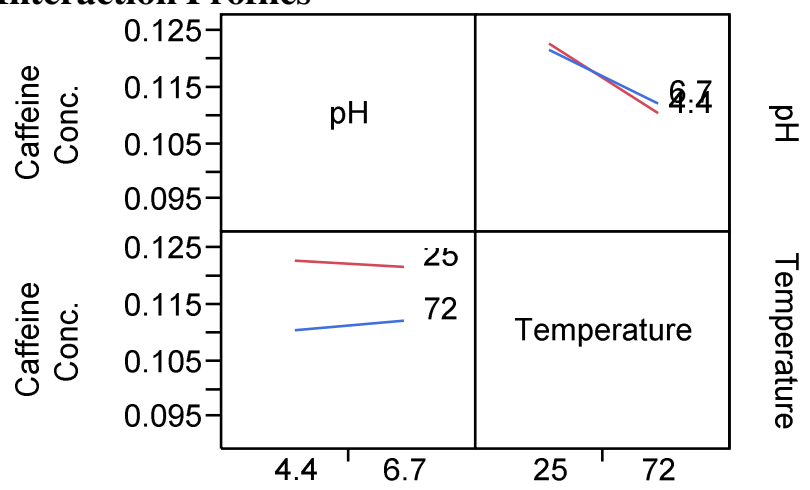




\section{Appendix C: Statistical analysis by JMP - binding 5-CGA to sodium}

\section{caseinate trials}

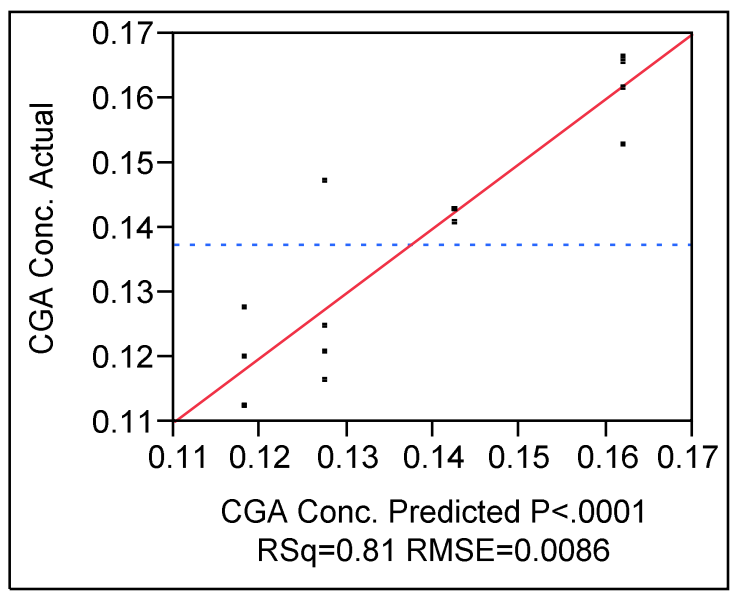

\section{Summary of Fit}

RSquare

RSquare Adj

Root Mean Square Error

Mean of Response

Observations (or Sum Wgts)

Parameter Estimates

Term

Intercept

$\mathrm{pH}[4.4]$

Temperature[25]

$\mathrm{pH}[4.4] *$ Temperature[25]
0.812019

0.765024

0.008564

0.1374

16

Estimate
0.1374
-0.002525
0.014675
-0.00715

Std Error
0.0014
0.002141
0.002141
0.002141

DFDen

\section{1}

t Ratio

98.14

$-1.18$

Prob $>|\mathbf{t}|$

11

11

11

$-3.34 \quad 0.0066^{*}$

Fixed Effect Tests

$\begin{array}{lrrrrr}\text { Source } & \text { Nparm } & \text { DF } & \text { DFDen } & \text { F Ratio } & \text { Prob }>\text { F } \\ \text { pH } & 1 & 1 & 11 & 1.3910 & 0.2631 \\ \text { Temperature } & 1 & 1 & 11 & 46.9847 & <.0001^{*} \\ \text { pH*Temperature } & 1 & 1 & 11 & 11.1535 & 0.0066^{*}\end{array}$


pH

Leverage Plot

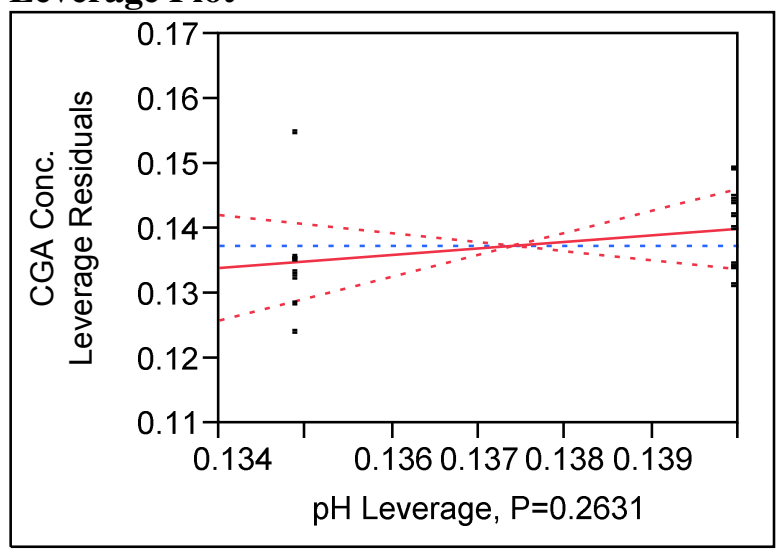

Least Squares Means Table

Level Least Sq Mean

Std Error

$4.4 \quad 0.13487500$

0.00255803

6.7

0.13992500

0.00255803

\section{LS Means Plot}

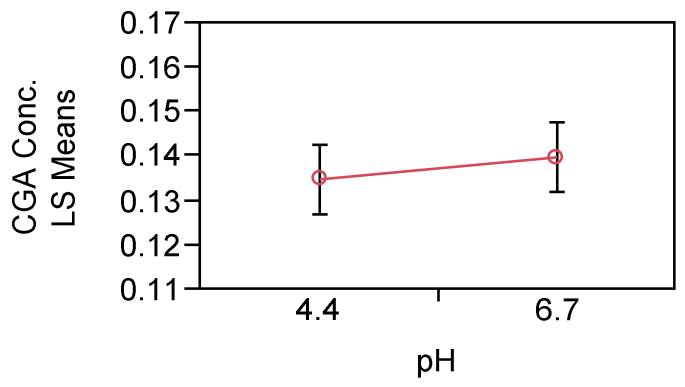

Page 110 


\section{LSMeans Differences Student's t}

$\alpha=0.017$

LSMean[i] By LSMean[j]

\begin{tabular}{|l|r|r|}
\hline $\begin{array}{l}\text { Mean[i]-Mean[j] } \\
\text { Std Err Dif } \\
\text { Lower CL Dif }\end{array}$ & 4.4 & 6.7 \\
Upper CL Dif & & \\
\hline 4.4 & 0 & -0.0051 \\
& 0 & 0.00428 \\
& 0 & -0.0171 \\
& 0 & 0.00702 \\
\hline 6.7 & 0.00505 & 0 \\
& 0.00428 & 0 \\
& -0.007 & 0 \\
& 0.01712 & 0 \\
\hline
\end{tabular}

Level

$\begin{array}{lll}6.7 & \text { A } & 0.13992500\end{array}$

$\begin{array}{lll}4.4 & \text { A } & 0.13487500\end{array}$

Levels not connected by same letter are significantly different.

\section{Temperature}

Leverage Plot

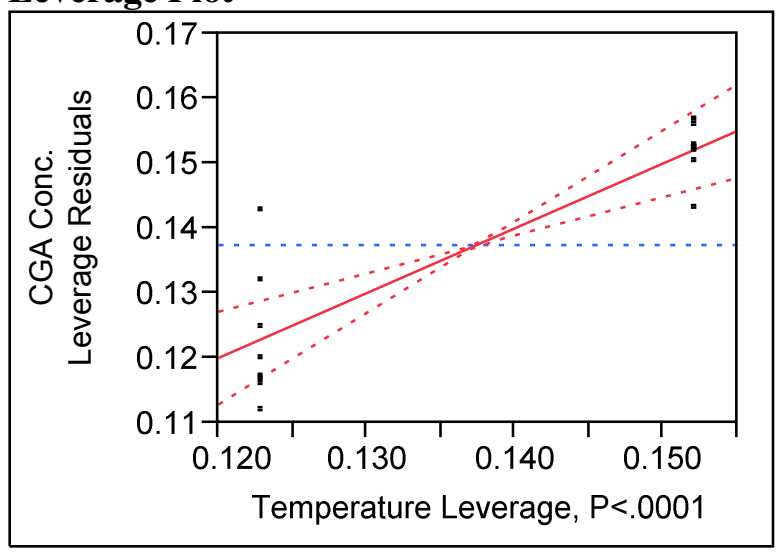

\section{Least Squares Means Table}

$\begin{array}{lrr}\text { Level } & \text { Least Sq Mean } & \text { Std Error } \\ 25 & 0.15207500 & 0.00255803 \\ 72 & 0.12272500 & 0.00255803\end{array}$

\section{LS Means Plot}

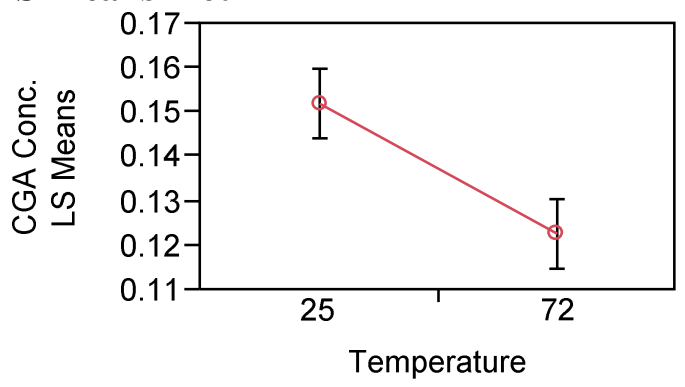

Page 111 


\section{LSMeans Differences Student's t}

$\alpha=0.017$

LSMean[i] By LSMean[j]

\begin{tabular}{|l|r|r|}
\hline $\begin{array}{l}\text { Mean[i]-Mean[j] } \\
\text { Std Err Dif } \\
\text { Lower CL Dif } \\
\text { Upper CL Dif }\end{array}$ & 25 & 72 \\
\hline 25 & 0 & 0.02935 \\
& 0 & 0.00428 \\
& 0 & 0.01728 \\
& 0 & 0.04142 \\
\hline 72 & -0.0294 & 0 \\
& 0.00428 & 0 \\
& -0.0414 & 0 \\
& -0.0173 & 0 \\
\hline
\end{tabular}

$\begin{array}{llllr}\text { Level } & & & \text { Least Sq Mean } \\ 25 & \text { A } & & 0.15207500 \\ 72 & & \text { B } & 0.12272500\end{array}$

Levels not connected by same letter are significantly different.

pH*Temperature

Leverage Plot

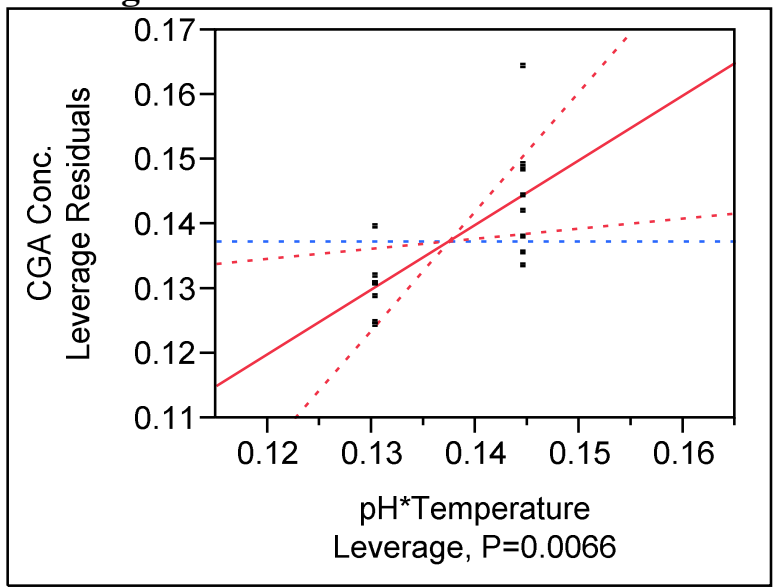

Least Squares Means Table

Level

$4.4,25$

$4.4,72$

$6.7,25$

$6.7,72$
Least Sq Mean

0.14240000

0.12735000

0.16175000

0.11810000
Std Error

0.00396366

0.00396366

0.00396366

0.00396366 


\section{LS Means Plot}

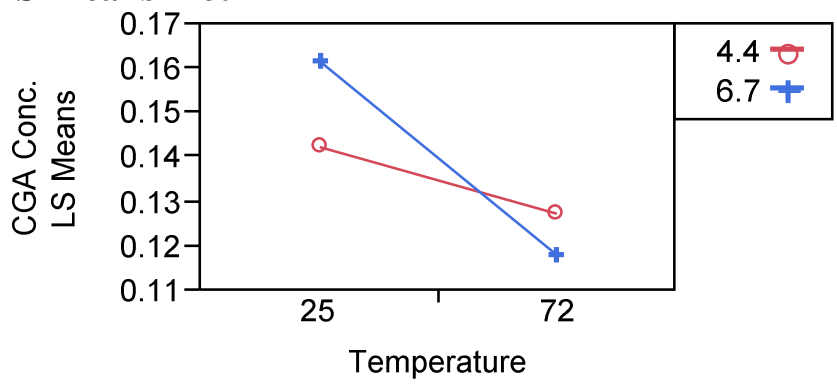

pH*Temperature

LSMeans Differences Tukey HSD

$\alpha=0.017$

LSMean[i] By LSMean[j]

\begin{tabular}{|l|r|r|r|r|}
\hline $\begin{array}{l}\text { Mean[i]-Mean[j] } \\
\text { Std Err Dif } \\
\text { Lower CL Dif } \\
\text { Upper CL Dif }\end{array}$ & $4.4,25$ & $4.4,72$ & $6.7,25$ & $6.7,72$ \\
\hline $4.4,25$ & & & & \\
& & & & \\
& 0 & 0.01505 & -0.0194 & 0.0243 \\
& 0 & -0.0071 & -0.0415 & 0.0021 \\
& 0 & 0.03725 & 0.00285 & 0.0465 \\
\hline $4.4,72$ & -0.0151 & 0 & -0.0344 & 0.00925 \\
& 0.00606 & 0 & 0.00606 & 0.00606 \\
& -0.0372 & 0 & -0.0566 & -0.0129 \\
& 0.00715 & 0 & -0.0122 & 0.03145 \\
\hline $6.7,25$ & 0.01935 & 0.0344 & 0 & 0.04365 \\
& 0.00606 & 0.00606 & 0 & 0.00606 \\
& -0.0028 & 0.0122 & 0 & 0.02145 \\
& 0.04155 & 0.0566 & 0 & 0.06585 \\
\hline $6.7,72$ & -0.0243 & -0.0092 & -0.0437 & 0 \\
& 0.00606 & 0.00606 & 0.00606 & 0 \\
& -0.0465 & -0.0314 & -0.0658 & 0 \\
& -0.0021 & 0.01295 & -0.0215 & 0 \\
\hline
\end{tabular}

Level

$6.7,25$

$4.4,25$

$4.4,72$

$6.7,72$
A

A B

B C

$\mathrm{C}$
Least Sq Mean

0.16175000

0.14240000

0.12735000

0.11810000

Levels not connected by same letter are significantly different.

\section{Day}

Least Squares Means Table

Level Least Sq Mean

$1 \quad 0.13552606$

$2 \quad 0.13927394$

Std Error

Page 113 


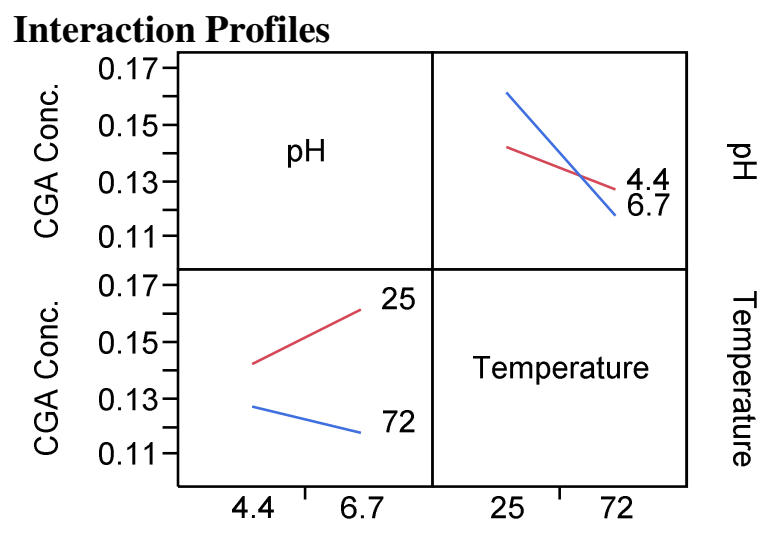

Page 114 
Appendix D: Statistical analysis by JMP - model assumptions (caffeine)

\section{Normality}

\section{Distributions}

Studentized Resid Caffeine Conc.

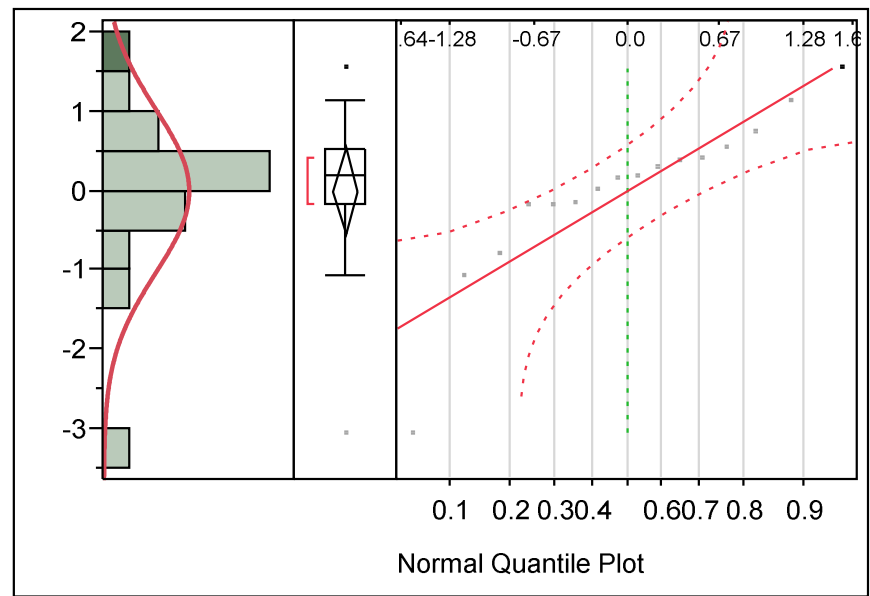

Normal(-4e-15,1.0328)

\section{Quantiles}

$\begin{array}{lcc}100.0 \% & \text { maximum } & 1.53725 \\ 99.5 \% & & 1.53725 \\ 97.5 \% & & 1.53725 \\ 90.0 \% & & 1.24456 \\ 75.0 \% & \text { quartile } & 0.51037 \\ 50.0 \% & \text { median } & 0.17217 \\ 25.0 \% & \text { quartile } & -0.1706 \\ 10.0 \% & & -1.6688 \\ 2.5 \% & & -3.0376 \\ 0.5 \% & & -3.0376 \\ 0.0 \% & & -3.0376\end{array}$

\section{Summary Statistics}

Mean

Std Dev

Std Err Mean

Upper $95 \%$ Mean

Lower $95 \%$ Mean

$\mathrm{N}$

Fitted Normal

\section{Parameter Estimates}

Type Parameter

Location

$\mu$

Dispersion
$-4.08 \mathrm{e}-15$

1.0327956

0.2581989

0.5503379

$-0.550338$

$-2 \log ($ Likelihood $)=45.4386494007571$

Upper $95 \%$

0.5503379

1.5984486

\section{Page 115}




\section{Equal Variances}

Residual by Predicted Plot

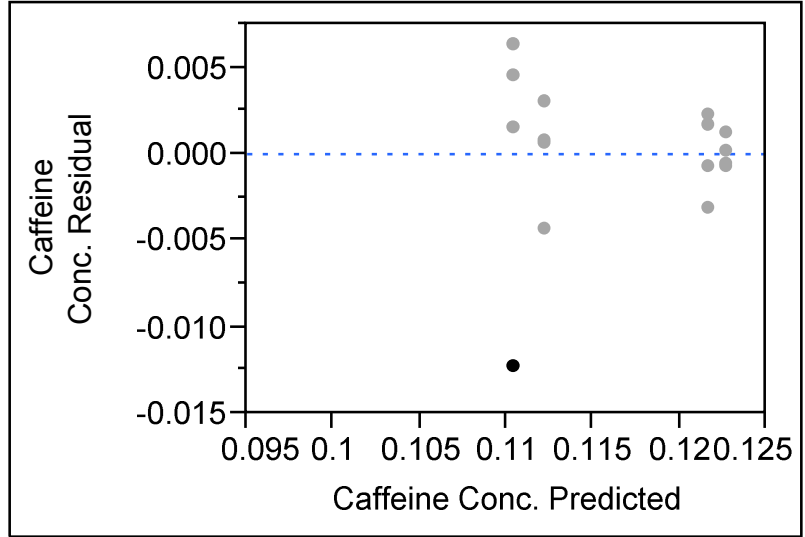

Page 116 
Appendix E: Statistical analysis by JMP - model assumptions (5-CGA)

\section{Normality}

\section{Distributions}

Studentized Resid CGA Conc.

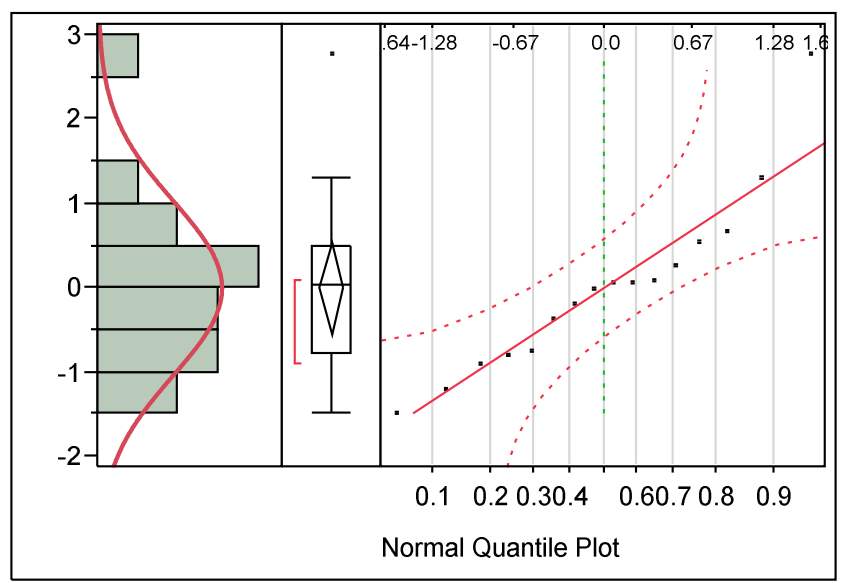

Normal(1.1e-15,1.0328)

\section{Quantiles}

$\begin{array}{lrr}100.0 \% & \text { maximum } & 2.75655 \\ 99.5 \% & & 2.75655 \\ 97.5 \% & & 2.75655 \\ 90.0 \% & & 1.73614 \\ 75.0 \% & \text { quartile } & 0.47497 \\ 50.0 \% & \text { median } & 0.02418 \\ 25.0 \% & \text { quartile } & -0.791 \\ 10.0 \% & & -1.2919 \\ 2.5 \% & & -1.4854 \\ 0.5 \% & & -1.4854 \\ 0.0 \% & \text { minimum } & -1.4854\end{array}$

\section{Summary Statistics}

$\begin{array}{lr}\text { Mean } & 1.055 \mathrm{e}-15 \\ \text { Std Dev } & 1.0327956 \\ \text { Std Err Mean } & 0.2581989 \\ \text { Upper 95\% Mean } & 0.5503379 \\ \text { Lower 95\% Mean } & -0.550338 \\ \mathrm{~N} & 16\end{array}$

Fitted Normal

Parameter Estimates

(eter

Estimate Lower 95\% Upper 95\%

$\begin{array}{lll}1.055 \mathrm{e}-15 & -0.550338 & 0.5503379\end{array}$

Dispersion $\quad \Sigma$

$\begin{array}{lll}1.0327956 & 0.7629311 & 1.5984486\end{array}$

$-2 \log ($ Likelihood $)=45.4386494007507$ 


\section{Equal Variances}

Residual by Predicted Plot

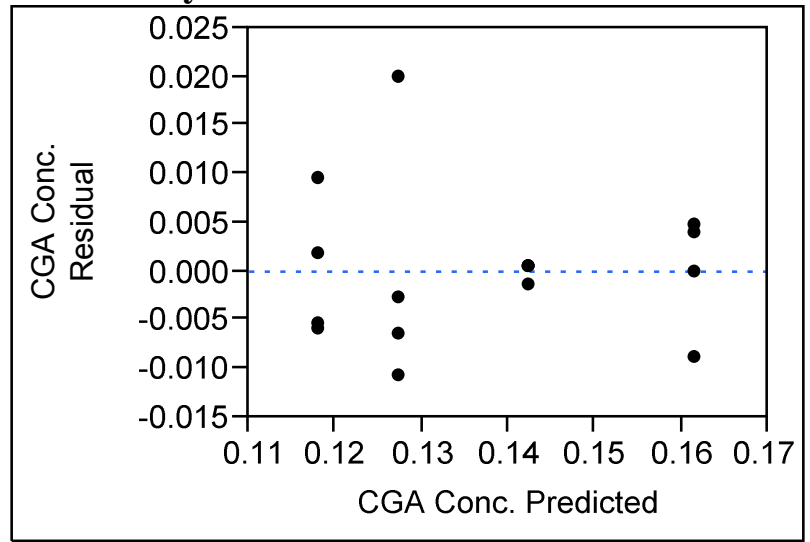

Page 118 